Reconhecimento de fala para navegação em aplicativos móveis para português brasileiro

Edwin Miguel Triana Gómez

DISSERTAÇÃO APRESENTADA

$\mathrm{AO}$

INSTITUTO DE MATEMÁTICA E ESTATÍSTICA

DA

UNIVERSIDADE DE SÃO PAULO

PARA

OBTENÇÃO DO TÍTULO

$\mathrm{DE}$

MESTRE EM CIÊNCIAS

Programa: Ciência da Computação

Orientador: Prof. Dr. Fabio Kon

Durante o desenvolvimento deste trabalho o autor recebeu auxílio financeiro do $\mathrm{CNPq}$

São Paulo - Brasil, julho de 2011 


\section{Reconhecimento de fala para navegação em aplicativos móveis para português brasileiro}

Esta dissertação contém as correções e alterações sugeridas pela Comissão Julgadora durante a defesa realizada por Edwin Miguel Triana Gómez em 17/06/2011.

O original encontra-se disponível no Instituto de Matemática e Estatística da Universidade de São Paulo.

Comissão Julgadora:

- Prof. Dr. Fabio Kon (orientador) - IME-USP

- Prof. Dr. Marcelo Finger - IME-USP

- Profa. Dra. Rebecca Anne Bates - MSU, Mankato, EUA 
aos meus pais Clelia e Jaime, aos meus irmãos e aos meus sobrinhos 


\section{Agradecimentos}

Antes de tudo, quero agradecer a Deus, que me colocou em uma família que sempre me tem apoiado no desenvolvimento dos meus projetos, e por ter me brindado a oportunidade de viajar ao Brasil para realizar o meu mestrado e me enriquecer tanto intelectualmente quanto culturalmente.

Muitas pessoas estiveram envolvidas antes e durante o desenvolvimento do meu trabalho de pesquisa para a obtenção do grau de mestre em Ciência da Computação no Instituto de Matemática e Estatística da Universidade de São Paulo. Inicialmente quero agradecer ao meu orientador, o professor Fabio Kon, quem foi meu primeiro contato com a USP, quem me ajudou no processo de admissão no programa e quem me acompanhou durante todo o desenvolvimento do mestrado.

O meu trabalho de mestrado não teria sido igual se não tivesse recebido o apoio constante da professora Rebecca Bates. A ela devo um muito obrigado!. Foi ela quem me introduziu no mundo do reconhecimento de fala, quem me apresentou conceitos fundamentais da área, assim como me orientou nas tarefas de pesquisa, experimentação e redação da dissertação. De novo, muito obrigado Becky.

Às varias pessoas que em algum momento estiveram envolvidas no desenvolvimento do meu mestrado, muito obrigado. Em especial quero agradecer aos amigos do IME, entre eles: Ana Paula, Claudia Melo, Edith Sonco, Alvaro Mamani, Felipe Besson, Gustavo Duarte, Jorge Guevara e William Colen, sua presença e apoio constante foi uma ajuda importante durante o alcance dos meus objetivos. Muito obrigado também aos professores e demais funcionários do IME e da USP em geral. Aos meus amigos e familiares na Colômbia que sempre estiveram comigo mesmo com a longa distância.

Finalmente quero agradecer a CNPq, FAPESP e Microsoft Research pelo apoio financeiro para o desenvolvimento da pesquisa, e à Nokia pelo fornecimento de um aparelho para realizar os testes e experimentos no meu projeto. 


\section{Resumo}

Os dispositivos móveis modernos estão equipados com componentes de alta capacidade que permitem o uso de aplicações sofisticadas como navegadores Web e planilhas eletrônicas. Graças à alta portabilidade dos dispositivos móveis, é possível usar ferramentas de software em cenários atípicos onde computadores convencionais não seriam apropriados. No entanto, estão presentes nesses dispositivos alguns problemas de usabilidade devido ao tamanho reduzido das telas e à falta de teclados mais acessíveis. Muitos componentes gráficos na mesma tela e esquemas de navegação complicados resultam em níveis de usabilidade baixos. Uma abordagem que tenta reduzir este impacto negativo é o uso de interfaces multimodais, tais como as de reconhecimento de fala. Nesse tipo de interface, o módulo de reconhecimento de fala coleta sinais de voz e os converte em cadeias de caracteres (representando palavras). Essas palavras podem ser usadas depois como comandos do sistema. Trabalhos recentes têm demonstrado que os dispositivos móveis atuais têm a capacidade de executar aplicações de reconhecimento de fala, apesar das capacidades de processamento inferiores.

O Borboleta é um sistema móvel de código aberto para telessaúde, usado na coleta de dados durante encontros domiciliares. Durante o encontro, o profissional da saúde revisa o estado do paciente e coleta a informação usando a interface do sistema. Se o profissional estiver concentrado apenas no paciente, ele poderá se esquecer de salvar informações importantes, e, se ele estiver concentrado na manipulação do sistema, poderá omitir aspectos fundamentais no atendimento ao paciente.

O objetivo do presente trabalho de pesquisa é reduzir o nível de atenção requerido para o uso do sistema Borboleta por meio de reconhecimento de fala na navegação através das funcionalidades do sistema, permitindo ao profissional dar maior atenção ao paciente. A metodologia de desenvolvimento do projeto inclui uma revisão bibliográfica para definir o estado da arte da área, uma pesquisa sobre o software disponível para reconhecimento de fala, uma coleta de dados dos comandos do sistema em português brasileiro para treinar e testar o sistema, uma etapa de projeção e desenvolvimento para definir a arquitetura de integração com o Borboleta, e uma fase de testes para medir a precisão do sistema e seus níveis de usabilidade e aceitação por parte do usuário.

Palavras-chave: reconhecimento de fala, interfaces multimodais, computação móvel. 


\begin{abstract}
Modern handheld devices are equipped with high capability components allowing the use of sophisticated applications such as Web browsers and spreadsheets. Because of low electricity dependency, mobile devices can take advantage of software tools in non-typical scenarios where desktop computers cannot operate. However, there are some usability issues with these devices because of their small screens and lack of keyboards. Many graphical components on small screens and complicated navigation schemas result in low user satisfaction. An approach to reduce this negative impact is the use of multimodal interfaces such as a spoken language interface. With this type of interface, a speech recognition module collects user utterances and converts them to word chains. These word chains can then be used as system commands. Recent work has shown that current mobile devices are able to execute speech recognition applications despite their lower processing abilities.

The Borboleta telehealth system is a open source mobile device application that supports data collection during residential visits carried out by a specialized healthcare team. During each visit, the healthcare professional checks the patient health status and collects information using the Borboleta interface. If the professional is focused just on the patient, he could forget to record important patient data, but if he is focused on the system operation, he could fail to get some relevant information from the patient.

The current document presents research that addresses the goal of reducing the user attention level required by Borboleta operation by providing speech recognition capabilities to augment navigation through the software functions, allowing the professional to pay more attention to the patient. The project methodology is composed of a bibliography revision to establish the state-of-the-art of the field, a review of available speech recognition software, data collection of Brazilian utterances to train and test the system, a design and development stage that defined the system architecture and integration with Borboleta and a testing process to measure the system accuracy, its usability and acceptance level.
\end{abstract}

Keywords: speech recognition, multimodal interfaces, mobile computing. 


\section{Resumen}

Los dispositivos móviles modernos están equipados con componentes de alta capacidad que permiten el uso de aplicaciones sofisticadas como navegadores Web y hojas de calculo. Gracias a su alta portabilidad, es posible usar en estos dispositivos herramientas de software en escenarios atípicos donde computadores convencionales no serian apropiados. Sin embargo, existen algunos problemas de usabilidad debido al tamaño reducido de las pantallas y a la falta de teclados mas accesibles. Muchos componentes gráficos en una misma pantalla y esquemas de navegación complicados resultan en niveles de usabilidad bajos. Un abordaje que busca reducir este impacto negativo es el uso de interfaces multimodales, tales como las de reconocimiento de voz. En este tipo de interfaces, el modulo de reconocimiento de voz captura señales sonoras y las convierte en cadenas de caracteres (representando palabras). Estas palabras pueden ser usadas posteriormente como comandos del sistema. Trabajos recientes han demostrado que los dispositivos móviles actuales están en capacidad de ejecutar aplicaciones de reconocimiento de voz, a pesar de las capacidades de procesamiento inferiores.

Borboleta es un sistema móvil de código abierto para telemedicina usado en la recolección de datos durante los encuentros domiciliares. Durante un encuentro, el profesional de la salud revisa el estado del paciente y recolecta la información usando la interfaz del sistema. Si el profesional se concentra solo en el paciente, podría olvidar guardar informaciones importantes, pero si al contrario, el profesional se concentra solo en la manipulación del sistema, podría omitir aspectos fundamentales durante la atención al paciente.

El objetivo del presente trabajo de investigación es reducir el nivel de atención requerido para el uso del sistema Borboleta por medio de reconocimiento de voz para la navegación a través de las funcionalidades del sistema, permitiendo al profesional dar mayor atención al paciente. La metodología de desarrollo del proyecto incluyó una revisión bibliográfica para definir el estado del arte del área, una investigación sobre el software disponible para reconocimiento de voz, una recolección de muestras de los comandos del sistema en portugues brasileño para entrenar y probar el sistema, una etapa de diseño y desarrollo que define la arquitectura e integración con Borboleta, y una fase de pruebas para medir la precisión del sistema y sus niveles de usabilidad y aceptación por parte del usuario.

Palavras-chave: reconocimiento de voz, interfaces multimodales, computación móvil. 


\section{Sumário}

Nomenclatura $\quad$ xi

Lista de Figuras xiii

Lista de Tabelas $\quad$ xv

I Dissertação 1

1 Introdução 2

1.1 Definição do problema . . . . . . . . . . . . . . . . . . . 3

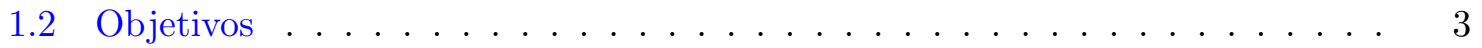

1.3 Organização da dissertação . . . . . . . . . . . . . . . . . . 4

2 Fundamentos teóricos 5

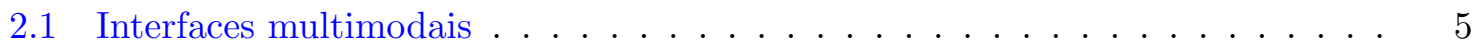

2.2 Reconhecimento de fala . . . . . . . . . . . . . . . . 6

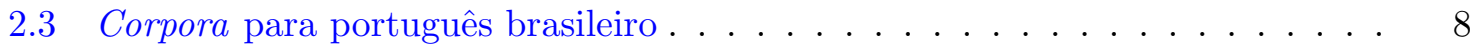

2.4 O aplicativo de telessaúde Borboleta . . . . . . . . . . . . 8

2.5 Dispositivos móveis . . . . . . . . . . . . . . . . . . 9

2.5.1 Reconhecimento de fala em dispositivos móveis . . . . . . . . . . . . 9

2.5.2 Software de reconhecimento de fala para dispositivos móveis . . . . . . 10

2.6 Trabalhos relacionados . . . . . . . . . . . . . . . . . . . . . . 11

3 Desenvolvimento do projeto $\quad 13$

3.1 Seleção do sistema de reconhecimento de fala . . . . . . . . . . . . . . . . 13

3.2 Desenvolvimento dos modelos acústicos e de linguagem . . . . . . . . . . . . . 14

3.2 .1 Definição de palavras-chave . . . . . . . . . . . . . . . . . . . . . 14

3.2 .2 Coleta de dados . . . . . . . . . . . . . . . . . . . . . 14

3.2 .3 Fase de treinamento . . . . . . . . . . . . . . . . . . 15

3.3 Projeto e desenvolvimento do software . . . . . . . . . . . . . . . 15

3.3.1 Migração do Pocketsphinx . . . . . . . . . . . . . . . . . . . 15

3.3.2 Serviço de reconhecimento de fala para dispositivos móveis . . . . . . 16

4 Avaliação $\quad \mathbf{2 0}$

4.1 Acurácia no reconhecimento de fala . . . . . . . . . . . . . . . . . . 20

4.1 .1 Descrição dos experimentos . . . . . . . . . . . . . . . . 20

4.1 .2 Resultados de acurácia . . . . . . . . . . . . . . . . . . . 21

4.2 Desempenho do sistema . . . . . . . . . . . . . . . . . . . 21

4.3 Usabilidade do sistema - Caso de estudo Borboleta . . . . . . . . . . . . . 23

4.3 .1 Resultados de usabilidade . . . . . . . . . . . . . . . . 23 
5 Discussão 28

5.1 Trabalhos futuros . . . . . . . . . . . . . . . . . . . . . . 29

II Apêndices - Relatório técnico (Inglês) 31

A Introduction $\mathbf{3 3}$

A.1 Problem statement . . . . . . . . . . . . . . . . . . . . . 33

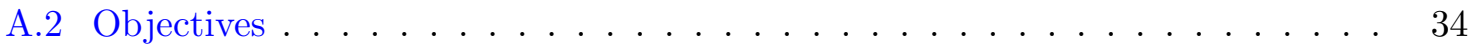

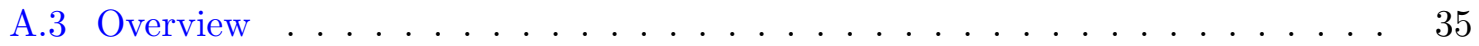

B Background $\quad 36$

B.1 Multimodal interfaces . . . . . . . . . . . . . . . . 36

B.1.1 Guidelines for multimodal interfaces design . . . . . . . . . . . . 37

B.2 Speech Recognition . . . . . . . . . . . . . . . . . . 38

B.2.1 History of speech recognition . . . . . . . . . . . . . . 38

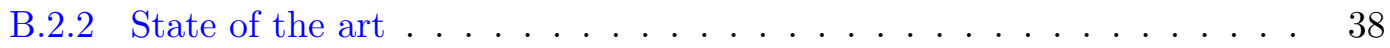

B.2.3 Concepts . . . . . . . . . . . . . . . . 40

B.3 Brazilian Portuguese corpora . . . . . . . . . . . . . . . . . 46

B.4 The Borboleta telehealth system . . . . . . . . . . . . . 46

B.5 Mobile devices . . . . . . . . . . . . . . . . . . . . . 47

B.5.1 Mobile devices and platforms . . . . . . . . . . . . . 48

B.5.2 Speech recognition on mobile devices . . . . . . . . . . . . . 50

B.5.3 Available ASR software for mobile devices . . . . . . . . . . . . . . 50

B.6 Related work . . . . . . . . . . . . . . . . . . . . . 52

B.6.1 VoiceAssist application . . . . . . . . . . . . . . . 52

B.6.2 The MONA project . . . . . . . . . . . . . . 53

B.6.3 The ACICARE platform . . . . . . . . . . . . . 53

B.6.4 MMI for navigation in complex mobile applications . . . . . . . . . 53

B.6.5 Parakeet . . . . . . . . . . . . . . . . . 53

C Project Development

C.1 Selection of the speech recognition system . . . . . . . . . . . . 55

C.2 Acoustic and language models development . . . . . . . . . . . . . 56

C.2.1 Data collection . . . . . . . . . . . . . . . . . 56

C.2.2 Training phase . . . . . . . . . . . . . . . . 58

C.3 Software design and development . . . . . . . . . . . . . . . . 60

C.3.1 Development environment . . . . . . . . . . . . . . . . . 61

C.3.2 Pocketsphinx porting. . . . . . . . . . . . . . . . 61

C.3.3 MSRS Architecture. . . . . . . . . . . . . . . 65

C.3.4 MSRS native (open $\mathrm{C} / \mathrm{C}++) \ldots \ldots \ldots \ldots 67 \ldots \ldots$

C.3.5 MSRS JavaMe side . . . . . . . . . . . . . . . . . . . . . . 69

C.3.6 C++/JavaMe interaction . . . . . . . . . . . . . . . . 71

C.3.7 Navigation commands structure . . . . . . . . . . . . . . . . . . . 74

$\begin{array}{ll}\text { D Evaluation } & \mathbf{7 9}\end{array}$

D.1 Speech recognition accuracy . . . . . . . . . . . . . . . . . . . . 79

D.1.1 Batch testing . . . . . . . . . . . . . . . . . . . . . 79

D.1.2 Live decoding testing . . . . . . . . . . . . . . . . . 80

D.1.3 Accuracy results . . . . . . . . . . . . . . . . . . . 80

D.1.4 Analysis of results . . . . . . . . . . . . . . . . 81

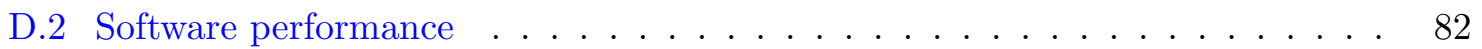


D.2.1 Performance results . . . . . . . . . . . . . . . . . . . . . . 83

D.2.2 Analysis of results . . . . . . . . . . . . . . . . 85

D.3 System usability - Borboleta case study . . . . . . . . . . . . . . 85

D.3.1 Experiment design . . . . . . . . . . . . . . . . . 86

D.3.2 Usability results . . . . . . . . . . . . . . . . . . . . . . . 89

D.3.3 Analysis of results . . . . . . . . . . . . . . . . . 89

E Discussion $\quad 92$

E.1 Future directions . . . . . . . . . . . . . . . . . . . . . . . . 93

$\begin{array}{lll}\text { F Acoustic and Language modeling } & 95\end{array}$

$\begin{array}{ll}\text { G IDEs description } & 97\end{array}$

G.1 Carbide.c $++\ldots \ldots \ldots \ldots \ldots$. . . . . . . . . . . . . . . . 97

G.2 QtCreator . . . . . . . . . . . . . . . . . . . 98

G.3 Netbeans . . . . . . . . . . . . . . . . . . . . . . . . . . . . . . . . . . 99

H MMP files for Sphinxbase and Pocketsphinx 102

$\begin{array}{ll}\text { I Protocol communication values } & 104\end{array}$

$\begin{array}{lll}\text { J Usability questionnaires } & 106\end{array}$

$\begin{array}{ll}\text { Referências Bibliográficas } & 107\end{array}$ 


\section{Nomenclatura}

API Application programming interface

ARM Advanced RISC machine

ASR Automatic speech recognition

CID Catálogo internacional de doenças

CPU Central processing unit

DARPA Defense Advanced Research Projects Agency

DSR Distributed speech recognition

ESR Embedded speech recognition

FSG Finite state grammar

GB Gigabyte

GPS Global positioning system

GSM Global system for mobile communications

GUI Graphical user interface

HMM Hidden Markov model

HTK Hidden Markov model toolkit

IDE Integrated development environment

IME Instituto de Matematica e Estatística

ISO International organization for stardarization

LPC Linear predictive coding

MB Megabyte

ME $\quad$ Mobile edition

MFCC Mel frequency cepstral coefficient

MIT Massachusetts Institute of Technology

MMI Multimodal interface

MONA Mobile multimOdal next generation applications

MPUQ Mobile phone usability questionnaire 
MSRS Mobile speech recognition service

MVC Model/View/Controller

NSR Network speech recognition

OGI Oregon Graduate Instituite

OS Operating system

PCM Pulse code modulation

PDA Personal digital assitant

RAM Random access memory

SER Sentence speech recognition

SR Speech recognition

SUS Sistema único de saúde

UFRGS Universidade federal do Rio grande do sul

USP Universidade de São Paulo

WAV Waveform file audio format

WER Word error rate

WIMP Windows, icons, menus and pointing device 


\section{Lista de Figuras}

2.1 Facilidades de entrada e saída em dispositivos móveis . . . . . . . . . . . 5

2.2 Processo de reconhecimento de fala . . . . . . . . . . . . . . . 6

2.3 Modelo fonte-filtro . . . . . . . . . . . . . . . . . . . 7

2.4 Arquitetura do sistema de telessaúde Borboleta . . . . . . . . . . . . 8

2.5 Borboleta - exemplo de telas do sistema . . . . . . . . . . . . . . 9

2.6 Abordagens para reconhecimento de fala em dispositivos móveis . . . . . . . . 10

3.1 Diagrama de navegação do Borboleta . . . . . . . . . . . . . . . . . . 14

3.2 Serviço de reconhecimento de fala - Arquitetura geral . . . . . . . . . . 17

3.3 Interface gráfica do módulo Qt/C++ do SpeechMe . . . . . . . . . . . . 18

3.4 Botão de ativação de reconhecimento - SpeechButton . . . . . . . . . . . . . 18

3.5 Quadro de diálogo durante reconhecimento de comando . . . . . . . . . . 19

4.1 Uso de memória e CPU . . . . . . . . . . . . . . . . . . . . . . . 22

4.2 Questionário de usabilidade - não multimodal . . . . . . . . . . . . . . . . 24

4.3 Redução de cliques e tempo . . . . . . . . . . . . . . . . . 26

4.4 Resultados de perguntas específicas . . . . . . . . . . . . . . 26

4.5 Resultados de perguntas gerais . . . . . . . . . . . . . . . 27

B.1 Input/Output facilities on mobile devices . . . . . . . . . . . . . . . 36

B.2 Speech recognition process . . . . . . . . . . . . . . . . . . 40

B.3 Speech recognition process . . . . . . . . . . . . . . . . . . . 41

B.4 ASR Back-end: recognition phase . . . . . . . . . . . . . . . . . 42

B.5 Dynamic Time Warping . . . . . . . . . . . . . . . . . . . 43

B.6 Markov generation model . . . . . . . . . . . . . . . . . 45

B.7 Borboleta - Overall architecture . . . . . . . . . . . . . . . 46

B.8 Borboleta - Interface screens . . . . . . . . . . . . . . . . . 47

B.9 Mobile OS world market Q1 $2011 \ldots \ldots \ldots \ldots \ldots$

B.10 Symbian architecture . . . . . . . . . . . . . . . . . . . 49

B.11 Java ME architecture . . . . . . . . . . . . . . . . . . . . . . 49

B.12 Speech recognition approaches for mobile devices . . . . . . . . . . . 51

B.13 VoiceAssist operations . . . . . . . . . . . . . . . . . 52

B.14 VoiceAssist interfaces . . . . . . . . . . . . . . . . . . . 52

B.15 AT\&T multimodal interface . . . . . . . . . . . . . . . . . . 54

B.16 Parakeet interface . . . . . . . . . . . . . . . . . . . 54

C.1 Borboleta navigation diagram . . . . . . . . . . . . . . 56

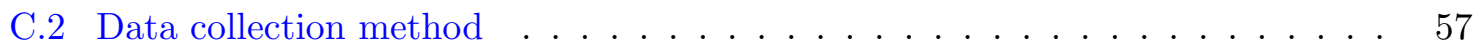

C.3 Language model example $(\mathrm{JSGF}) \ldots \ldots \ldots \ldots$. . . . . . . . . . . 58

C.4 Porting process . . . . . . . . . . . . . . . . . 62

C.5 Mobile Speech Recognition Service on Symbian architecture . . . . . . . . . . 66

C.6 Mobile Speech Recognition Service - General Architecture . . . . . . . . . . 66 
C.7 SpeechMe GUI - Qt/C+t . . . . . . . . . . . . . . . . . . 68

C.8 Speech Me architecture - C+t side . . . . . . . . . . . . . . . . 69

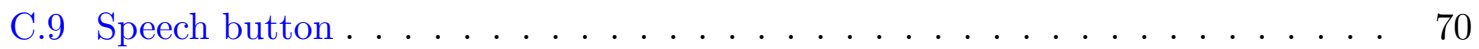

C.10 SpeechDialog view . . . . . . . . . . . . . . . . . . 70

C.11 Speech configuration view - Java ME . . . . . . . . . . . . . . . 71

C.12 Speech me - Architecture - Java ME . . . . . . . . . . . . . . . . 72

C.13 Registry client . . . . . . . . . . . . . . . . . 73

C.14 Decoding process . . . . . . . . . . . . . . . . . . . 73

C.15 Remove client . . . . . . . . . . . . . . . . . . . . . . . . 74

C.16 Borboleta navigation diagram - Initial . . . . . . . . . . . . . 75

C.17 Borboleta navigation diagram - Final . . . . . . . . . . . . 76

C.18 Random navigation . . . . . . . . . . . . . . . . . . . 77

C.19 Navigation diagram contexts . . . . . . . . . . . . . . . 78

C.20 Commands Structure . . . . . . . . . . . . . . . . . . 78

D.1 Nokia Energy Profiler . . . . . . . . . . . . . . . . . . . . . . . . . 82

D.2 Memory usage for SpeechMe and Borboleta . . . . . . . . . . . . 83

D.3 CPU usage for Speech Me and Borboleta . . . . . . . . . . . . . . . 84

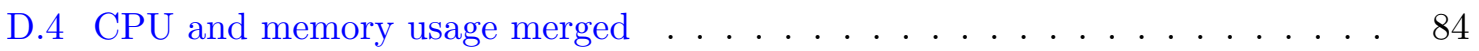

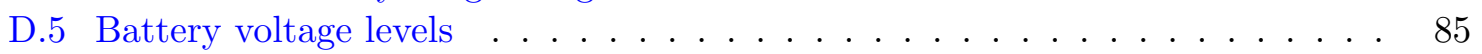

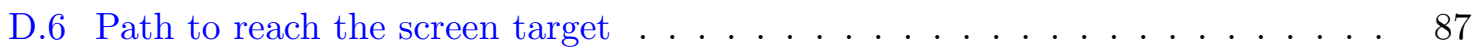

D.7 Navigation schema - Borboleta project . . . . . . . . . . . . 87

D.8 Clicks and time reduction $\ldots \ldots \ldots \ldots \ldots$

D.9 Specific questions results . . . . . . . . . . . . . . . . . . . . . . . . . . . 91

D.10 General questions results . . . . . . . . . . . . . . . . . . . . 91

F.1 Language model for individual words . . . . . . . . . . . . . . . . 96

G.1 Carbide IDE - New project wizard . . . . . . . . . . . . . . . . 98

G.2 Symbian emulator . . . . . . . . . . . . . . . . . . . . . . . . . 99

G.3 QtCreator IDE . . . . . . . . . . . . . . . . . . . . . . . . 100

G.4 Netbeans IDE . . . . . . . . . . . . . . . . . . . . . . 100

G.5 Java ME emulator . . . . . . . . . . . . . . . . . . . . . . . . 101

J.1 Pre-installation usability questionnaire - English _ . . . . . . . . . . 106 


\section{Lista de Tabelas}

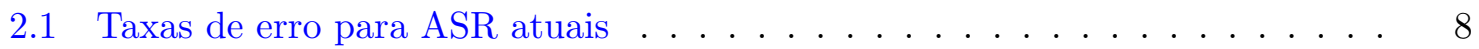

3.1 Palavras-chave para navegação no Borboleta . . . . . . . . . . . . . . . . . . . 14

4.1 Experimentos de acurácia - Testes automáticos . . . . . . . . . . . . 20

4.2 Resultados de acurácia - Testes automáticos . . . . . . . . . . . . . 21

4.3 Resultados de acurácia - Laboratório . . . . . . . . . . . . . . . . 21

4.4 Lista de tarefas - teste de usabilidade . . . . . . . . . . . . . . . . . 23

4.5 Resultados de teste de usabilidade - Perguntas específicas - Não multimodal . 25

4.6 Resultados de teste de usabilidade - Perguntas gerais - Não multimodal . . . 25

4.7 Resultados de teste de usabilidade - Perguntas específicas - Multimodal . . . 25

4.8 Resultados de teste de usabilidade - Perguntas gerais - Multimodal . . . . . . 25

B.1 Current recognition word error rates on desktop ASR systems . . . . . . . 40

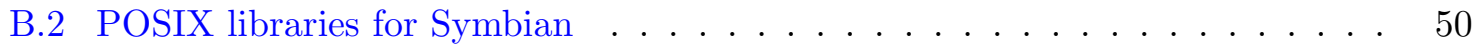

C.1 Keyword list for navigation on Borboleta . . . . . . . . . . . 57

C.2 Dictionary example . . . . . . . . . . . . . . . . . . . 59

C.3 Command recognition - Approach 1 example . . . . . . . . . . . . . 59

C.4 Command recognition - Approach 2 example . . . . . . . . . . . . . . 59

C.5 Command recognition - Approach 3 example . . . . . . . . . . . . . 60

C.6 Command recognition - Approach 4 example . . . . . . . . . . . . . . . 60

D.1 Accuracy experiments - Batch testing . . . . . . . . . . . . . 80

D.2 Accuracy results - Batch decoding . . . . . . . . . . . . . . . 81

D.3 Accuracy results - Live decoding in Lab. . . . . . . . . . . . . . . . . 81

D.4 Response time - decoding performance . . . . . . . . . . . . . . . 84

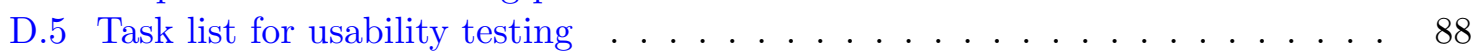

D.6 Usability testing results - Specific questions - Non-multimodal . . . . . . . . . 89

D.7 Usability testing results - General questions - Non-multimodal . . . . . . . . 89

D.8 Usability testing results - Specific questions - Multimodal . . . . . . . . . . 90

D.9 Usability testing results - General questions - Multimodal . . . . . . . . . . 90

F.1 Phonemes for Command words . . . . . . . . . . . . . . . . 95

I.1 Decoding states . . . . . . . . . . . . . . . . . . . . . . . . . 104

I.2 Response control values - prefixes . . . . . . . . . . . . . . . . . . . 104

I.3 Decoding states . . . . . . . . . . . . . . . . . . . . . . 105 


\section{Parte I}

\section{Dissertação}




\section{Capítulo 1}

\section{Introdução}

As novas tecnologias usadas nos dispositivos móveis têm aumentado sua gama de uso. Inicialmente, dispositivos móveis eram usados como telefones celulares, blocos de notas ou diretórios de contatos. As novas características incluem o uso de processadores de alto desempenho permitindo interfaces gráficas de alta qualidade e execução de tarefas complexas, ambientes de desenvolvimento de alto nível, como Java ME ou $\mathrm{C}++$, conexões sem fio de alta velocidade ( $\mathrm{Wi}-\mathrm{Fi}$ ), sistemas de posicionamento global e telas multitoque. Ao mesmo tempo, novo software tem sido desenvolvido; desde pequenas aplicações como reprodutores multimídia até as mais complexas, como navegadores Web ou jogos em 3D. No entanto, as telas de pequeno porte têm sido uma desvantagem no uso de aplicações sofisticadas. Muitas funções em uma tela pequena ou várias alterações na janela para concluir uma tarefa resultam em baixos níveis de aceitação por parte do usuário [34]. As interfaces multimodais são uma solução para esse problema. Este tipo de interfaces oferecem métodos alternativos de entrada/saída, como reconhecimento de fala, para a interação homem-computador. Nessas interfaces, os métodos de entrada como telas sensíveis ao toque, botões e interfaces de voz permitem o desenvolvimento de telas mais limpas e níveis de usabilidade mais altos.

Um aplicativo especializado é o sistema Borboleta, um projeto Java ME para coleta de informações médicas para apoio a programas de atenção básica domiciliar do sistema de saúde brasileiro (SUS) [13]. O Borboleta é desenvolvido na Universidade de São Paulo, no Centro de Competência em Software Livre ${ }^{1}$. Dado que o Borboleta oferece diferentes funcionalidades para o suporte dos encontros domiciliares, é importante que a interação com o dispositivo seja de boa qualidade, com uma estrutura bem definida, usando atalhos, menus, retroalimentação ao usuário (por exemplo, quadros de diálogo ou sons) e reconhecimento de fala para a execução de comandos.

Uma vantagem de se usar a voz como método de entrada é que a voz é uma habilidade natural do ser humano e não requer muita atenção ou observação direta, permitindo uma maior atenção para outras atividades [47]. Outras vantagens são a redução do uso das mãos e interação através do teclado para inserir informação. Isso permite melhor acesso para pessoas deficientes. Não obstante, usar voz como método de entrada traz alguns desafios, tais como lidar com o ruído do ambiente, sotaques regionais, grandes vocabulários, microfones de baixa qualidade e uso de dispositivos de baixa capacidade de processamento. Nos últimos 20 anos, sistemas de reconhecimento de fala robustos têm tratado esses problemas. Dois exemplos de código aberto são o HTK [68] e o Sphinx 4 [65], os quais têm um desempenho muito aceitável com taxas de erro baixas [54]. No entanto, esses sistemas foram desenvolvidos para computadores de mesa com recursos de processamento grandes. Em dispositivos móveis, os recursos de processamento e memória são limitados devido à dependência constante da bateria, tornando a implementação de sistemas de reconhecimento de fala ainda mais difícil.

\footnotetext{
${ }^{1}$ http://ccsl.ime.usp.br/borboleta.
} 


\subsection{Definição do problema}

Os dispositivos móveis oferecem a facilidade de acessar informação rapidamente, em qualquer momento, em praticamente qualquer lugar. Essa disponibilidade motiva os pesquisadores e desenvolvedores na implementação de novas aplicações. O objetivo dessas implementações é fornecer ferramentas úteis para acessar informação em ambientes de trabalho onde computadores normais não podem ser utilizados facilmente. Questões tais como tamanho do dispositivo e a necessidade de estar ligado na energia elétrica fazem dos computadores de mesa inadequados para várias tarefas. Os dispositivos móveis podem ser usados em lugares pouco comuns, como na coleta de informações em ambientes exteriores.

No campo dos dispositivos móveis, aplicações complexas ou sofisticadas exigem o foco e a atenção do usuário para produzir entradas de dados corretas ou para obter as saídas do aplicativo. No entanto, quando um dispositivo móvel é utilizado em conjunto com outras tarefas importantes, isto pode ter efeitos prejudiciais tanto na tarefa desempenhada quanto na manipulação do dispositivo. Um exemplo é o uso de GPS para a obtenção de indicações enquanto se está dirigindo em uma grande cidade, o sistema pode requer a entrada do endereço de destino e a leitura do resultado em uma tela de cinco polegadas. Uma abordagem que busca reduzir esse tipo de problema é o uso de interfaces multimodais [47], onde diferentes métodos de entrada e saída são utilizados para reduzir o nível de atenção do usuário, tornando o dispositivo mais fácil para usar. Um exemplo é o uso de reconhecimento de fala em conjunto às telas sensíveis ao toque como um método de entrada e a síntese de voz para indicar saídas do sistema.

O Borboleta é um sistema móvel para coleta de dados sobre a saúde de pacientes que requer um nível de atenção considerável. Durante um encontro domiciliar, médicos ou enfermeiros revisam as condições de saúde do paciente e, ao mesmo tempo, inserem informação no dispositivo. Se, durante o encontro, o profissional estiver focado na operação do sistema, ele pode ignorar informações importantes do paciente. Por outro lado, se ele estiver focado no paciente, ele pode manipular incorretamente o aplicativo e registrar informações erradas. Adicionalmente, os encontros domiciliares têm limite de tempo devido a compromissos do paciente e outros encontros agendados para o mesmo dia. A coleta de dados deve ser feita rapidamente mas, ao mesmo tempo, os dados coletados do paciente devem ser precisos e úteis.

\subsection{Objetivos}

Três objetivos foram definidos no começo do projeto. O primeiro objetivo foi verificar se a introdução de voz como método alternativo de entrada para navegação no aplicativo melhora a usabilidade do sistema Borboleta. Se assim for, os profissionais poderiam focar mais no cuidado do paciente e menos na manipulação do dispositivo. Atualmente, o Borboleta conta com uma interface gráfica de usuário complexa devido às múltiplas funcionalidades que oferece. Essa interface é composta por aproximadamente 25 telas que contêm diferentes componentes gráficos, tais como abas, campos de texto, listas e caixas de seleção e menus específicos do sistema, além de um esquema de navegação entre janelas. Para acessar uma funcionalidade específica no sistema é necessário conhecer a rota completa para alcançá-la. Tal rota pode ter duas ou mais janelas intermediárias, aumentado o tempo de operação para uma tarefa individual. Uma possível solução é o uso de atalhos para ir para janelas específicas, mas isso requer o aumento de elementos de interação como menus ou botões. A inclusão de novos botões ou menus aumenta a quantidade de elementos para ser apresentados na pequena tela.

O segundo objetivo foi usar um vocabulário reduzido em português para controlar o sistema Borboleta e abrir o caminho para o reconhecimento de fala para a coleta de anotações 
médicas com um vocabulário maior e mais especializado. Este objetivo foi definido pelo fato que o reconhecimento de fala na língua inglesa tem sido amplamente estudado em trabalhos anteriores, mas para português brasileiro poucas iniciativas têm sido apresentadas. Recentemente alguns esforços têm surgido no fornecimento de ferramentas para reconhecimento de fala para português brasileiro, entre eles a iniciativa FalaBrasil [44]. A maioria desses projetos é baseada em vocabulários de uso comum. No domínio médico, não existem projetos relevantes que usem português brasileiro para reconhecimento de fala.

O terceiro e último objetivo foi establelecer qual é comportamento das abordagens de reconhecimento de comandos para navegação, usando fonemas ou palavras completas, no sistema Borboleta. e qual oferece melhores resultados. Cada uma dessas abordagens apresenta tanto vantagens quanto desvantagens dependendo do domínio do problema. Em trabalhos anteriores, foram implementados alguns sistemas de reconhecimento de fala para inglês para dispositivos móveis [31, 70], mas a maioria deles tem uma arquitetura de reconhecimento distribuído, onde o processamento do sinal acontece no dispositivo móvel e o reconhecimento de fala é levado a cabo em um servidor remoto. Para navegação no sistema, todos os cálculos devem ser feitos no dispositivo móvel; no caso do projeto Borboleta, os dispositivos móveis não têm conexão com nenhuma rede durante os encontros médicos. Para a implementação de um sistema automático de reconhecimento de fala (Automatic speech recognition - ASR) em plataformas móveis foi avaliado se os dispositivos móveis atualmente utilizados no projeto podem levar a cabo tarefas de reconhecimento de fala e se essa abordagem melhora a experiência do usuário durante a manipulação do dispositivo móvel.

\subsection{Organização da dissertação}

A primeira parte da dissertação consiste em uma breve revisão bibliográfica que abrange interfaces multimodais, reconhecimento de fala, computação móvel e uma descrição do sistema Borboleta, seguida por uma seção de desenvolvimento do projeto, que mostra a nossa abordagem para tratar a questão da utilidade de uma interface multimodal para o sistema Borboleta, e apresenta a arquitetura do sistema proposto. Em seguida, uma seção de avaliação é apresentada, onde os resultados de precisão no reconhecimento de fala e os resultados de testes de usabilidade e desempenho do software são analisados. A primeira parte é concluída com uma discussão dos resultados e as implicações futuras deste trabalho. A segunda parte consiste em um relatório técnico em inglês sobre o desenvolvimento do projeto. O relatório apresenta de forma mais ampla os fundamentos teóricos e um maior detalhe na metodología de desenvolvimento do projeto. 


\section{Capítulo 2}

\section{Fundamentos teóricos}

O processo do reconhecimento de fala incorpora conceitos das ciências teóricas e aplicadas, incluindo processamento de sinais, processos estocásticos, algoritmos de busca e engenharia de software. Esta seção apresenta uma revisão dos conceitos básicos sobre interfaces multimodais, reconhecimento de fala e o estado da arte nesta área. Em seguida, apresentamos uma descrição dos corpora de voz (conjuntos de texto falado com transcrições - plural de corpus) existentes para português brasileiro e a apresentação do sistema Borboleta, usado neste trabalho. Depois é apresentada uma revisão das plataformas móveis disponíveis e seus ambientes de desenvolvimento. Finalizamos com uma discussão sobre os trabalhos relacionados.

\subsection{Interfaces multimodais}

Os sistemas multimodais processam um ou mais métodos de entrada para responder a uma requisição do usuário. O processamento pode ser combinado para responder uma só requisição, para resolver situações de ambiguidade [46], ou para oferecer modos de entrada alternativa [60]. Os métodos de entrada mais comuns são o teclado, o mouse, canetas digitais e telas sensíveis ao toque, mas também reconhecimento de fala, acelerômetros e gestos. A Figura 2.1 apresenta as diferentes facilidades para entrada/saída em dispositivos móveis. As entradas multimodais são frequentemente usadas com saídas multimídia, tais como interfaces visuais ou síntese de voz.

O objetivo das interfaces multimodais (multimodal interfaces - MMI) é melhorar a interação homem/computador fazendo uso de interfaces mais ricas em conteúdo e de formas de comunicação mais naturais [19]. Em contraste com as interfaces comuns, onde a requisição do usuário é facilmente determinada pela entrada, por exemplo, um botão clicado ou uma tecla pressionada, em MMI as entradas frequentemente misturam fluxos de sinais (áudio ou vídeo) que devem ser processados e interpretados com um grau de incerteza devido aos processos probabilísticos envolvidos [19].

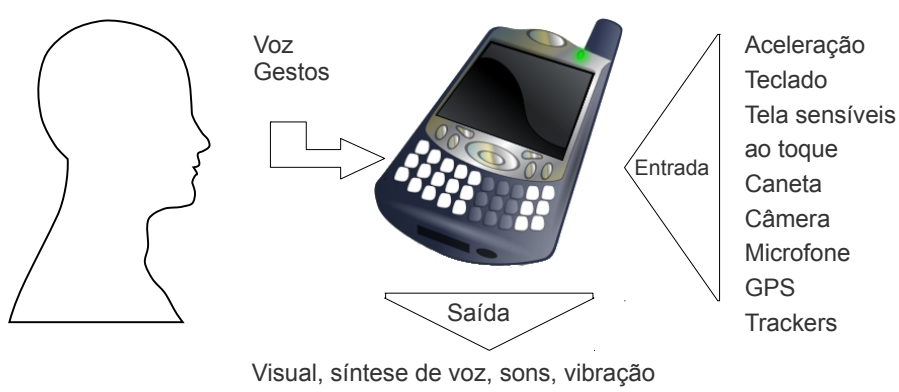

Figura 2.1: Facilidades de entrada e saída em dispositivos móveis 
De acordo com Oviatt [46], existem dois modos de entradas multimodais: o modo ativo, onde a requisição ou comando é dado ao computador de forma intencional, por exemplo um comando de voz ou um gesto na tela, e o modo passivo, onde o sistema captura o comportamento ou as ações do usuário para serem usadas como entradas sem ter sido expressados explicitamente. Expressões faciais e movimentos do corpo são exemplos de entradas passivas. Da mesma forma, as interfaces multimodais podem ser classificadas em três grupos. O caso mais simples ocorre quando modos alternativos de entrada (unimodal) para a execução de uma tarefa são oferecidos dando mais opções ao usuário. Se a entrada é composta de modos ativos e passivos, o sistema usa uma entrada mista (blended), por exemplo, usando voz e movimentos dos lábios. O caso mais complexo é com interfaces multimodais temporalmente em cascata (temporally-cascade MMI). Aqui o processamento de duas ou mais entradas é feito em forma sequencial, de modo que uma entrada restringe a interpretação da entrada seguinte.

Uma das vantagem que as MMIs trazem é o melhoramento da interpretação das requisições do usuário misturando informações parciais das diferentes fontes de entrada. Os sistemas podem também ser personalizados ou adaptados a diferentes usuários ou contextos em uma forma mais transparente. O uso de MMIs não é necessariamente mais eficiente que as interfaces WIMP (Windows, Icons, Menu, Pointing device). Dumas et al. [19], afirma que só se tem $10 \%$ de melhora na conclusão de tarefas. No entanto, usando MMIs, os usuários apresentaram $36 \%$ menos erros e mais de $95 \%$ dos usuários preferiram a interação multimodal sobre a unimodal. Essa redução na taxa de erro poderia ser derivada da carga de trabalho cognitiva baixa descrita pelo modelo de memória de curto prazo de Baddeley e Hitch [2]. Este modelo afirma que uma pessoa que executa duas tarefas simultâneas poderia ser eficiente em ambas, se cada uma delas pertence a um domínio perceptual diferente (exemplo: tarefas visuais e verbais). Isto ocorre porque dois dos três principais componentes da memória de curto prazo são dedicados ao processamento auditivo-verbal e ao visuo-espacial, enquanto que o outro componente atua como controlador do fluxo da informação. Algumas recomendações para o projeto de MMI são apresentadas na Seção B.1.1.

\subsection{Reconhecimento de fala}

O Reconhecimento automático de fala é um processo onde a voz é convertida para texto com um significado equivalente. Esse texto pode ser composto por dígitos, uma palavra ou frases e pode ser usado como um comando de execução na elaboração de documentos, ou em sistemas de diálogo automático ou de compreensão da fala. A evolução histórica dos sistemas de reconhecimento de fala é apresentada na Seção B.2.1.

Um sistema típico de reconhecimento de fala é composto por duas fases, o front-end e o back-end (Figura 2.2). O módulo front-end processa o sinal de voz e obtém os vetores de observação que são usados para treinamento e reconhecimento [4]. O módulo back-end é encarregado da busca da palavra misturando informações dos modelos acústicos e de linguagem extraídas da fase de treinamento.
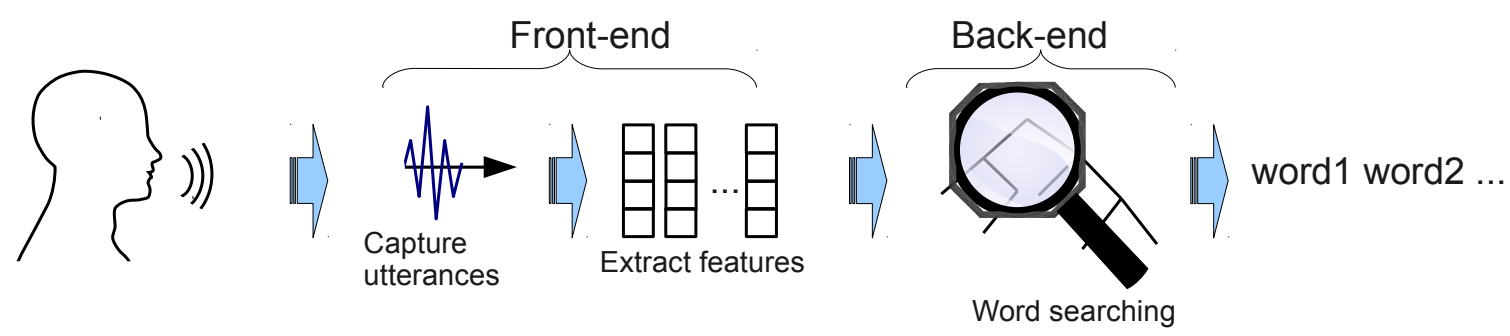

Figura 2.2: Processo de reconhecimento de fala 


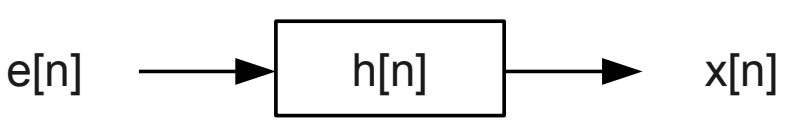

Figura 2.3: Modelo fonte-filtro

Uma medida comum para avaliar a precisão no reconhecimento de fala é a taxa de erro por palavras (word error rate - WER) apresentado na Equação 2.1.

$$
W E R=100 \% * \frac{S u b s+\text { Dels }+ \text { Ins }}{\# \text { de palavras na sentença correta }}
$$

onde $S u b s$ é a quantidade de substituições para uma palavra correta, Dels são as palavras omitidas e Ins são as palavras inseridas [27].

No front-end, os sinais de voz são convertidos para ondas digitais e posteriormente transformados em uma representação paramétrica para posterior processamento. Diferentes abordagens são utilizadas para obter a representação paramétrica. A análise de banco de filtros [49], linear predictive coding (LPC) [49] e coeficientes cepstrais em frequência Mel ( Mel frequency cepstral coefficients - MFCCs) [27] têm sido técnicas eficientes na extração de características de sinais de voz.

A caracterização do sinal é baseada no modelo fonte-filtro (source-filter), um modelo de produção de voz onde a voz $x[n]$ é o resultado da interação do ar dos pulmões (fonte ou excitação $e[n]$ ) com o trato vocal (filtro $h[n]$ ) através do tempo [27]. A Figura 2.3 apresenta o modelo fonte-filtro. O objetivo nesta etapa é separar a fonte e o filtro de modo que a informação do filtro possa ser usada no reconhecimento. Diferentes técnicas para estimar o filtro são baseadas principalmente em modelos de produção de voz, tais como o banco de filtros e o LPC, e modelos de percepção de fala, como MFCC. Quando o filtro é estimado, o inverso do filtro é usado sobre o sinal para obter a fonte [27].

Uma vez que o sinal é processado, o passo seguinte é reconhecer o sinal baseado em um treinamento prévio. No treinamento, exemplos de voz são utilizados para extrair as caraterísticas para criar modelos que depois são usados na busca de novos sinais. O treinamento depende da abordagem de reconhecimento, onde podem ser requeridos a geração de templates (template matching) ou modelos acústicos (exemplo: modelos acústicos de Markov), em conjunto com modelos de linguagem (gramáticas de estado finito ou modelos $N$-gram). Em reconhecimento, o sistema executa um processo de mapeamento para obter a palavra mais próxima ao sinal de entrada. Uma revisão mais profunda sobre os conceitos de reconhecimento de fala é apresentada na Seção B.2.3.

Na tabela 2.1 são apresentadas as taxas de erro típicas no reconhecimento automático de fala para Inglês no 2007 [54]. Na tabela, Connected digits são cadeias de dígitos gravados em um ambiente sem ruídos, resource management (da DARPA) inclui comandos militares, consultas e respostas em um ambiente sem ruídos. e Wall Street Journal inclui sons de leituras de notícias de jornais em um ambiente sem ruídos, com um vocabulário de 5.000 palavras. O corpus SWITCHBOARD é um conjunto de gravações de conversações espontâneas em um ambiente telefônico, o qual dificulta a tarefa de reconhecimento. Os melhores WERs para tarefas típicas neste campo estão ainda ao redor de $20 \%$ [20,26] dependendo de um conjunto particular para testes e das abordagens para modelagem. Para pequenos vocabulários (exemplo: dígitos conectados) e um ambiente sem ruídos, as taxas de erro estão perto de $1 \%$. O WER cresce quando são usados vocabulários maiores, fala espontânea ou não planejada, vários locutores e ruídos no ambiente. 
Tabela 2.1: Taxas de erro para sistemas de reconhecimento automático de fala atuais [20, 26, 54]

\begin{tabular}{|l|c|c|}
\hline Tarefa & Tamanho do vocabulário & WER (\%) \\
\hline Connected digits & 11 & 0.6 \\
\hline Resource Management & 1.000 & 2.7 \\
\hline Wall Street Journal & 5.000 & 7.2 \\
\hline SWITCHBOARD & $30 \mathrm{~K}-40 \mathrm{~K}$ & $\approx 20$ \\
\hline
\end{tabular}

\subsection{Corpora para português brasileiro}

Para levar a cabo o processo de treinamento de testes no sistema, um conjunto de gravações de voz e suas transcrições devem estar disponíveis para uma língua específica. Para corpora em português brasileiro existem poucos recursos disponíveis, mas atualmente alguns esforços têm aumentado esses recursos. Spoltech é um corpus de português brasileiro com 8.119 gravações desenvolvido pelo Oregon Graduate Insitute (OGI) e pela Universidade do Rio Grande do Sul (UFRGS) [15]. OGI-22 é outro corpus disponível que é constituído por gravações telefônicas em 22 línguas [14]. Este corpus é menor e só poucas gravações têm transcrição. Trabalhos anteriores com estes dois corpora na Universidade Federal de Pará apresentaram WER de 29\%, 20.1\% e 29.3\% usando Julius, HDecode e IBM ViaVoice com modelos independentes [43]. Outro corpus multilíngua é o Globalphone, da Universidade de Karlsruhe [61]. Para português brasileiro, o corpus foi criado usando 102 locutores lendo notícias do jornal Folha de São Paulo. VoxForge é uma iniciativa recente para disponibilizar recursos para reconhecimento de fala na internet [63]. Neste caso, diferentes corpora e modelos acústicos são disponibilizados com licença GPL. Para português, um conjunto de gravações com um total de 51 minutos encontra-se disponível. Todos os corpora disponíveis são compostos de vocabulários de palavras de uso comum. Muitas das gravações têm sido feitas usando endereços, números telefônicos e informações pessoais. Ainda que a abrangência de usuários seja grande, o conjunto de palavras não atende às necessidades do Borboleta, que usa um vocabulário voltado para a área médica.

\subsection{O aplicativo de telessaúde Borboleta}

Borboleta é uma aplicação de código aberto usada na coleta de informação médica durante os encontros domiciliares no programa de saúde domiciliar do Centro de Saúde Escola Samuel Pessoa, da Faculdade de Medicina da USP [17, 13]. A Figura 2.4 apresenta a arquitetura geral do sistema Borboleta.

Antes do encontro domiciliar, o Borboleta é sincronizado com o sistema central SAGUISaúde, onde as informações médicas dos encontros agendados são transferidas no dispositivo. Durante o encontro, os dados são salvos no dispositivo. Uma vez que os médicos estão no centro

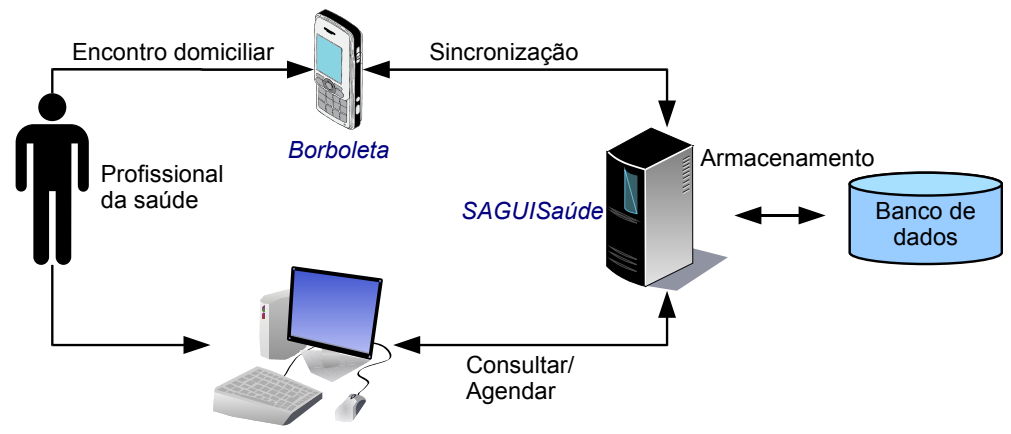

Figura 2.4: Arquitetura do sistema de telessaúde Borboleta 
de saúde, a informação coletada é enviada ao servidor para depois ser acessada via navegador Web. O sistema Borboleta foi desenvolvido em Java ME com o intuito de garantir uma maior compatibilidade com as plataformas móveis. O sistema pode ser executado em Windows Mobile, Palm OS, Symbian e Android, mas as funcionalidades de multimídia só estão disponíveis, no momento, para dispositivos Nokia (Symbian S60), devido a que estes ofereciam um melhor hardware para processamento multimídia e a API Java suportava o acesso a estas funcionalidades. A Figura 2.5 apresenta algumas das telas do sistema utilizadas durante o encontro domiciliar.

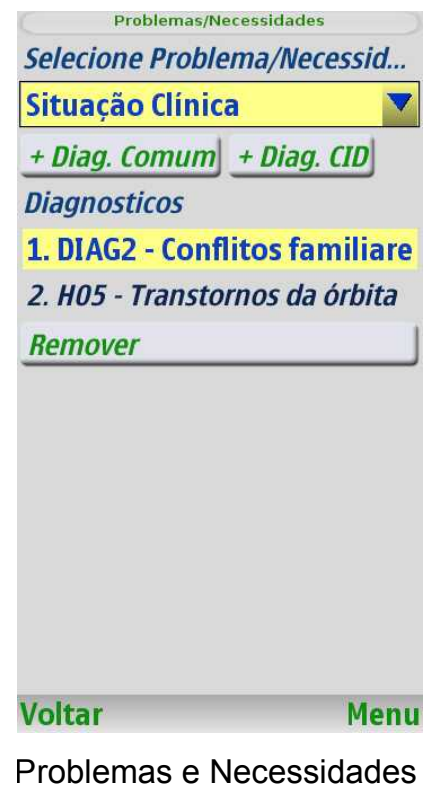

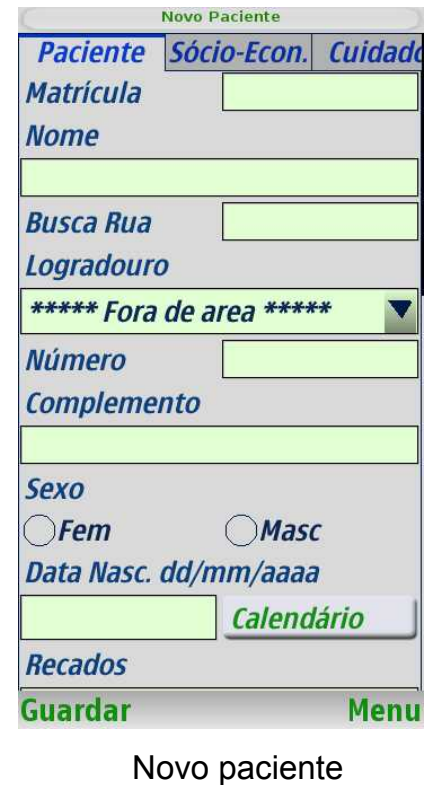

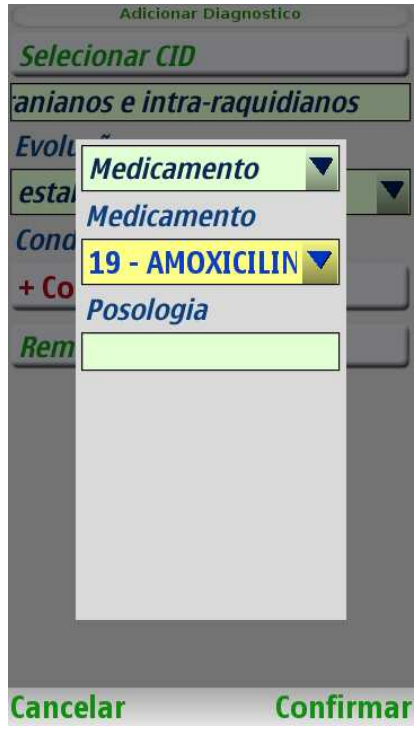

Dados específicos

Figura 2.5: Borboleta - exemplo de telas do sistema

\subsection{Dispositivos móveis}

Os dispositivos móveis são aparelhos eletrônicos portáteis que podem trabalhar em diversos ambientes. Normalmente, esses dispositivos possuem menor tamanho e peso do que os computadores normais. No nosso caso, foram examinados dispositivos compatíveis com o Borboleta, isto é, PDAs e telefones celulares com Java Mobile Edition (Java ME). Esses dispositivos são equipados com processadores com velocidades entre $300 \mathrm{MHz}$ e $1 \mathrm{GHz}$, RAM de $32 \mathrm{MB}$ até $512 \mathrm{MB}$ e armazenamento de $512 \mathrm{MB}$ até $32 \mathrm{~GB}$. Para entrada/saída, os dispositivos tipicamente contam com telas sensíveis ao toque de 4", miniteclados qwerty, acelerômetro, GPS e câmera. Tipicamente, as plataformas móveis são compatíveis com um conjunto específico de dispositivos. Isso causa uma dependência do dispositivo com o sistema operacional e portanto, também dos aplicativos desenvolvidos para essa plataforma. Uma abordagem para reduzir essa dependência é o uso de uma máquina virtual como Java. O Borboleta foi desenvolvido com Java ME para reduzir essa dependência e manter uma maior compatibilidade entre as diferentes plataformas móveis. A Seção B.5 apresenta uma descrição detalhada das plataformas móveis, focando-se na arquitetura da plataforma Symbian.

\subsubsection{Reconhecimento de fala em dispositivos móveis}

Dado que o processo de reconhecimento de fala é divido em duas fases, ele pode ser desacoplado. Existem três possíveis abordagens para a implementação de reconhecimento de fala automático (automatic speech recognition - ASR) em dispositivos móveis: reconheci- 
mento de fala embutido (embedded speech recognition - ESR), reconhecimento de fala em rede (network speech recognition - NSR) e reconhecimento de fala distribuído (distributed speech recognition - DSR) [69]. Um ESR (Figura 2.6.a) contém todas as fases do ASR no dispositivo móvel. Esses sistemas geralmente são providos de vocabulários pequenos, mas não precisam de conexões externas. No NSR (Figura B.12.b), o front-end e o back-end são localizados no servidor remoto e o dispositivo móvel simplesmente envia o sinal para ser processado no servidor. A principal vantagem do NSR é que o servidor conta com maiores recursos de processamento e memória, o que permite o uso de vocabulários maiores e taxas de erro menores. Nos sistemas DSR (Figura B.12.c), o front-end é embutido no dispositivo móvel e o back-end é localizado em um servidor externo. O dispositivo obtém os vetores de observação e os envia ao servidor onde a busca é realizada. A vantagem do DSR sobre o NSR é que a transmissão dos vetores de observação requer menos banda do que a transmissão de sinais completos.

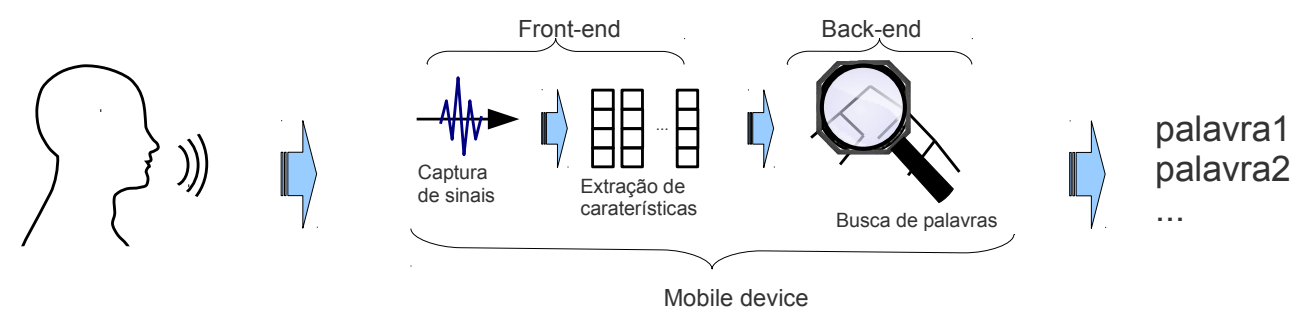

(a) Reconhecimento de fala embutido - ESR

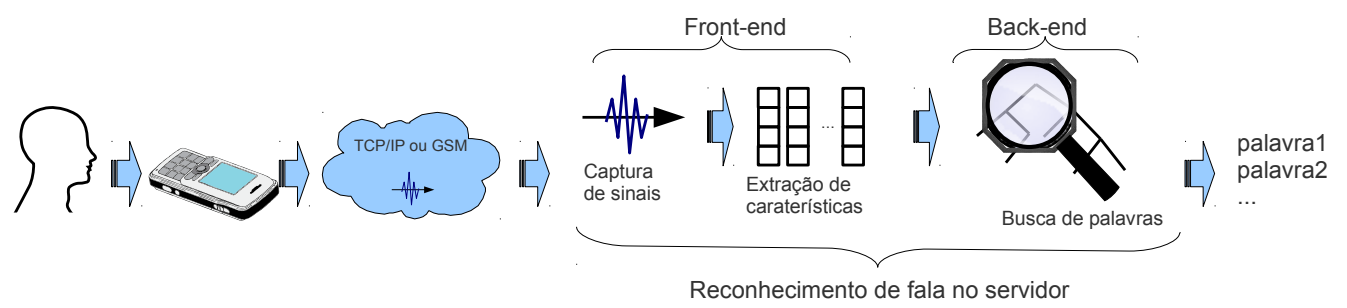

(b) Reconhecimento de fala em rede - NSR
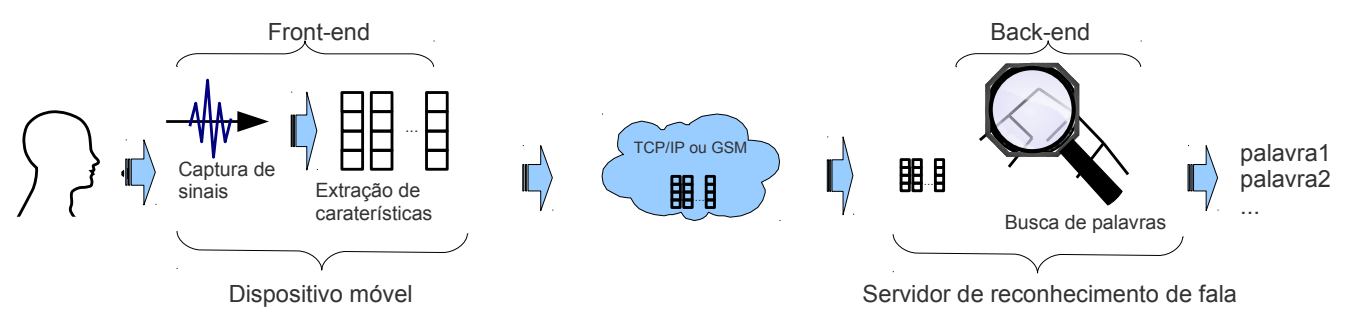

(c) Reconhecimento de fala distribuído - DSR

Figura 2.6: Abordagens para reconhecimento de fala em dispositivos móveis

\subsubsection{Software de reconhecimento de fala para dispositivos móveis}

Atualmente, os dispositivos móveis são equipados com melhores recursos de processamento e armazenamento que os antigos. Esse fato incrementa o interesse dos pesquisadores no desenvolvimento de aplicações sofisticadas. Pocketsphinx e PocketSUMMIT são duas aplicações para reconhecimento de fala projetadas para ser executadas em dispositivos móveis.

Pocketsphinx é um sistema para reconhecimento de fala contínua desenvolvido pelo Carnegie Mellon University Language Technology Institute [29]. O sistema foi baseado em Sphinx-II [28]. Para ser executado em dispositivos móveis, foram realizadas otimizações no uso da memória e no acesso linear a dados da CPU, foram implementadas operações em pontofixo, dado que as operações de ponto-flutuante eram geralmente emuladas nos dispositivos 
móveis e reduzem o desempenho do aplicativo. Também foram modificados alguns algoritmos de reconhecimento, especialmente na extração de caraterísticas, cálculos de Gaussianas e avaliação dos HMM. Um caso de sucesso usando Pocketsphinx em dispositivos móveis é o projeto Parakeet, um aplicativo para reconhecimento de fala contínua que é executado em dispositivos móveis baseados em Linux (Maemo) [62].

PocketSUMMIT é um sistema de reconhecimento de fala contínua desenvolvido pelo MIT Computer Science and Artificial Intelligence Laboratory [24]. O sistema é baseado no SUMMIT, um sistema de reconhecimento para computadores de mesa [59]. As optimizações nesse aplicativo incluem: compressão 8:1 para a quantização de parâmetros da Gaussiana e a redução do transdutor de estados finitos usando técnicas de poda e simplificação do decodificador para um grafo estado/palavra na primeira passada da busca.

\subsection{Trabalhos relacionados}

Os recentes avanços em tecnologias de software e hardware para dispositivos móveis têm permitido que sejam desenvolvidas aplicações móveis mais elaboradas usando interfaces multimodais, aumentando o escopo de aplicação a campos inexplorados. Esta seção apresenta uma breve revisão dos trabalhos relacionados ao uso de interfaces multimodais, com ênfase no uso de reconhecimento de fala. Uma revisão mais ampla é apresentada na Seção B.6.

\section{VoiceAssist}

A aplicação VoiceAssist foi desenvolvida por Samsung Electronics em colaboração com o Instituto Politécnico Rensselaer em 2006 [37]. O sistema é configurado como um sistema ESR com suporte para inglês dos Estados Unidos e é executado no sistema operacional Palm. A aplicação usa interface de voz para acessar diferentes funcionalidades do dispositivo, como a ligação por nome, e inicio e controle de outras aplicações. O VoiceAssist oferece quatro modos de operação: comandos de discagem (funções de chamada), aplicações de gravação, aplicações externas (execução) e aplicações do telefone (funções de verificação). As taxas de erro nos testes do aplicativo foram de $98.6 \%$ e $99.6 \%$ para comandos com ruído de sala e de carro.

\section{O projeto MONA}

O projeto MONA (Mobile multimOdal Next generation Applications) é um servidor de apresentação desenvolvido no Telecommunications Research Center of Vienna em 2004. O aplicativo permite a implantação de aplicações com funcionalidade de entrada e saída de voz de forma independente do dispositivo [1]. A ideia geral é desenvolver a interface de usuário uma vez só para diferentes plataformas móveis. O servidor MONA renderiza a interface multimodal em um dispositivo específico quando o cliente conecta-se via GSM ou rede sem fio. O sistema usa uma abordagem similar à dos navegadores web para criar as interfaces gráficas e uma configuração NSR para garantir a compatibilidade com um amplo intervalo de dispositivos. Devido à abordagem NSR utilizada, é necessária uma conexão contínua à rede.

\section{Plataforma ACICARE}

O ACICARE é uma plataforma para projeto de interfaces multimodais que permite o desenvolvimento rápido de MMI e facilita a avaliação dessas interfaces [55]. O ACICARE é baseado no ICARE, uma plataforma para projeto de MMI para computadores de mesa, e no ACIDU, uma ferramenta para obter informações sobre funcionalidades utilizadas e para avaliar as MMI. O ACICARE foi desenvolvido para ser executado em telefones baseados 
em Windows CE e foi testado no telefone SPV C500. A plataforma ACICARE pode ser integrada com funcionalidades do telefone como o gestor de contatos. Para reconhecimento de fala foi utilizado um sistema ESR comercial desenvolvido pela Fonix Speech Inc.

\section{MMI para navegação em aplicações móveis complexas}

Zhang et al. apresentam um estudo comparativo de uso de voz e de interfaces gráficas para navegação em aplicações com visualizações complexas em dispositivos móveis [71]. A avaliação foi levada a cabo usando um navegador Web para dispositivos móveis (Visualizer) em um iPhone, com o software AT\&T WATSON ASR como sistema de reconhecimento de fala. Zhang afirma que não existe muita diferença na efetividade usando a voz como método de entrada e usando a interface gráfica, mas essa pequena diferença poderia aumentar com a complexidade da tarefa. Zhang afirma que o uso de voz como método de entrada é mais eficiente e que na maioria dos casos os usuários preferem usar a voz ao invés da interface gráfica.

\section{Parakeet}

Parakeet é um sistema para reconhecimento de fala contínua para dispositivos móveis desenvolvido no Laboratório Cavendish na Universidade de Cambridge [62]. A aplicação é executada em dispositivos móveis baseados em Linux (Maemo) e usa Pocketsphinx como sistema de reconhecimento de fala [29], com suporte para inglês dos Estados Unidos e do Reino Unido. Além do reconhecimento de fala, o Parakeet fornece uma interface de correção que apresenta as possíveis palavras em ordem de probabilidade. O sistema apresenta taxas de erro de $16.2 \%$ e $25.6 \%$ em ambientes interiores e externos, respectivamente. Usando a interface de correção, o erro é reduzido para $1.2 \%$ e $2.2 \%$, respectivamente. 


\section{Capítulo 3}

\section{Desenvolvimento do projeto}

Para alcançar o objetivo de navegação no sistema Borboleta usando reconhecimento de fala, a seguinte metodologia foi definida: iniciou-se com uma avaliação do software de reconhecimento de fala existente, depois foram definidas as palavras chave e foi realizada uma coleta de dados para treinamento e testes. Em seguida, a arquitetura do sistema e a integração com o Borboleta foram projetadas e implementadas. O processo foi finalizado com uma fase de testes onde foram avaliados o desempenho do sistema, a acurácia, o grau de usabilidade e o nível de aceitação por parte do usuário. Esta seção descreve cada uma destas etapas.

\subsection{Seleção do sistema de reconhecimento de fala}

O objetivo principal nesta fase foi selecionar um sistema ASR que pudesse ser integrado ao sistema Borboleta. O processo de avaliação dos sistemas foi levado a cabo da seguinte forma:

Durante o processo de revisão foram avaliadas a robustez e acurácia do sistema de reconhecimento, a compatibilidade com dispositivos móveis, arquitetura do software, disponibilidade do código fonte, licença e grau de atividade da comunidade de desenvolvimento. Robustez e acurácia são importantes dado que a precisão no reconhecimento de comandos depende disso. A compatibilidade está relacionada com a facilidade de migração e integração com o sistema Borboleta. Dada a natureza do Borboleta, tivemos como requisitos o uso de tecnologias de código aberto, licença compatível com a BSD e que o projeto estivesse em desenvolvimento ativo.

Dado o amplo intervalo de dispositivos móveis e as plataformas existentes, a compatibilidade do sistema foi restrita ao conjunto de dispositivos usados no projeto Borboleta. Como o Borboleta tinha sido desenvolvido em Java ME, duas opções de integração foram avaliadas: primeiro, o desenvolvimento de um módulo completo de reconhecimento de fala em Java e, segundo, a migração de um sistema em $\mathrm{C}++$ existente para a plataforma Symbian onde o sistema seria executado de forma nativa. Devido à complexidade do desenvolvimento de um ASR do zero, optou-se pela migração de um sistema e a integração com o Borboleta via sockets.

Dois sistemas foram avaliados baseados no parâmetros anteriores, Pocketsphinx [29] e PocketSUMMIT [24] (ver descrição em Seção 2.5.2). Ao final, o Pocketsphinx foi selecionado devido ao contínuo desenvolvimento de projeto, disponibilidade do código, compatibilidade de licença e a migrações anteriores para outras plataformas móveis, tais como iPhone OS e Android. 


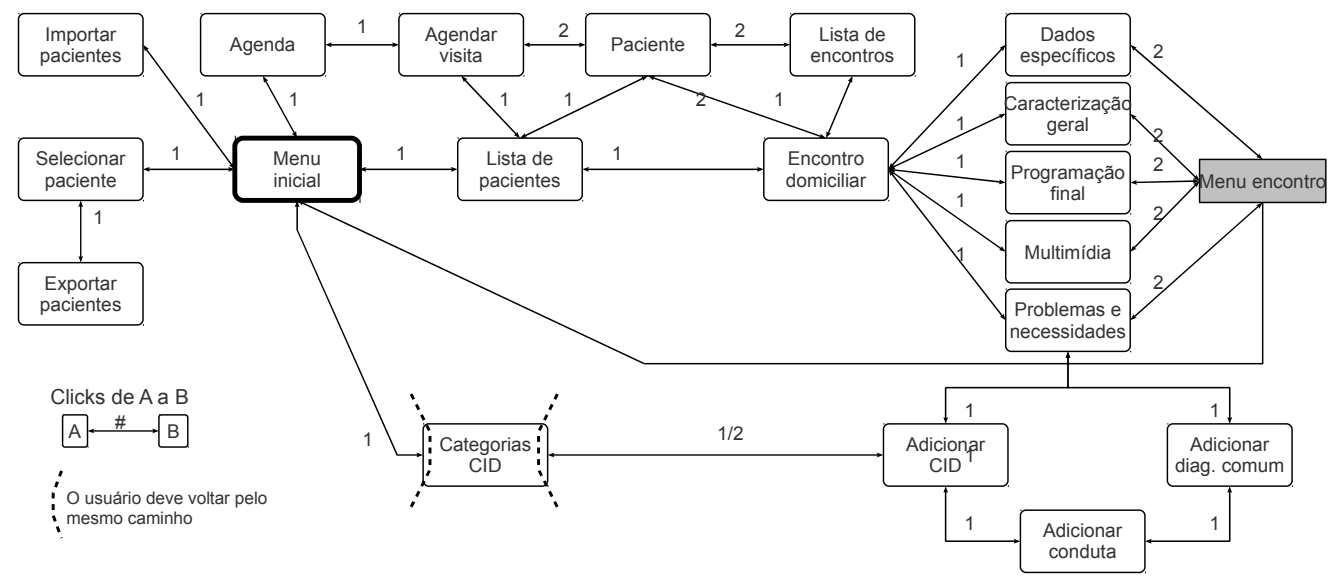

Figura 3.1: Diagrama de navegação do Borboleta

\subsection{Desenvolvimento dos modelos acústicos e de linguagem}

O processo de desenvolvimento dos modelos acústicos e de linguagem incluiu a definição dos comandos, coleta de dados e treinamento dos modelos acústicos. A seguir são descritas cada uma dessas etapas.

\subsubsection{Definição de palavras-chave}

Palavras-chave são palavras que o sistema reconhece como comandos. O conjunto de palavras-chave não foi composto por todas as funcionalidades do sistema, mas sim pelas quais o usuário precisava realizar mais de duas ações, tais como cliques ou seleção em menus de opções. A Figura 3.1 apresenta o diagrama de navegação do sistema Borboleta. Este diagrama define os caminhos possíveis para as funções principais do sistema. O valor de cada aresta está definido como o custo de passar de uma janela para outra (vértices), isto é, a quantidade de ações que o usuário deve realizar para acessar uma função específica. Baseados no diagrama, os caminhos com valor de dois ou mais formaram o grupo de comandos do sistema. A Tabela 3.1 relaciona os 15 comandos definidos para o sistema.

Tabela 3.1: Palavras-chave para navegação no Borboleta

\begin{tabular}{|l|l|l|}
\hline \multicolumn{3}{|c|}{ Comando } \\
\hline Menu inicial & Dados específicos & Caracterização geral \\
\hline Problemas e necessidades & Programação final & Encontros realizados \\
\hline Encontro & Novo encontro & Paciente \\
\hline Agenda & Agendar visita & Catálogo CID \\
\hline Sair & Voltar & Fechar \\
\hline
\end{tabular}

\subsubsection{Coleta de dados}

Para treinar os modelos acústicos e de linguagem e para testar o sistema, um conjunto de gravações com suas respectivas transcrições foi coletado. A equipe de profissionais da saúde do Borboleta é composta por oito pessoas, dois homens e seis mulheres. Cada membro da equipe realizou 75 gravações correspondentes a cinco grupos de gravações das 15 palavraschave. No total, 600 gravações foram coletadas para serem usadas em treinamento e testes. Cada amostra foi coletada usando o formato WAV com codificação PCM, com $16 \mathrm{KHz}$ de 
taxa de amostragem e precisão de 16 bits [11]. De acordo com Huang [27], uma amostragem de $16 \mathrm{KHz}$ reduz os erros em $20 \%$ em comparação com uma amostragem de $8 \mathrm{KHz}$, que é a amostragem típica para telefonia.

\subsubsection{Fase de treinamento}

Setenta porcento dos dados coletados foram usados para treinar o sistema. Nessa fase, foram gerados os modelos acústicos para as diferentes abordagens de modelagem: comandos isolados, modelos de palavra-inteira (whole-word) e modelos de palavras baseados em fonemas (phone-based). Para o modelo da linguagem, que define como os comandos devem estar formatados para serem aceitos pelo sistema, foi usado uma gramática de estados finitos (finite state grammar - FSG) baseada no esquema de navegação do sistema.

Com o intuito de definir qual abordagem apresentava os melhores resultados de acurácia, quatro combinações de modelagem de palavras e de linguagens foram testados. Exemplos destas abordagens são apresentados na Seção C.2.2.

\section{Abordagens para modelagens acústica e de linguagem}

1. Palavra-inteira/Comando composto (Whole-word/Composed command): o termo palavrainteira indica que as palavras no dicionário não são decompostas em fonemas, mas ficam como palavras completas. Os comandos nessa abordagem são constituídos por cadeias de palavras. Por exemplo, o comando NOVO ENCONTRO tem duas palavras no dicionário e o modelo da linguagem deve excluir as cadeias de palavras que não correspondem a esse modelo.

2. Fonema/Comando composto (Phoneme/Composed command): nessa abordagem, as palavras no dicionário são decompostas em fonemas. O modelo da linguagem apresenta a mesma estrutura que a abordagem anterior. Por exemplo, o comando NOVO ENCONTRO tem duas palavras decompostas no dicionário $(\mathrm{NOVO}=\mathrm{n}$ o $\mathrm{v} \mathrm{u}-\mathrm{ENCONTRO}=\mathrm{e} \sim$ $\mathrm{k} \mathrm{o} \sim \mathrm{tr} \mathrm{u}$ ) e o modelo da linguagem só aceita cadeias similares a esta.

3. Comando-inteiro/Palavra-inteira (Whole-command/whole word): nesse caso, o dicionário contém cada comando como uma cadeia de palavras. O modelo da linguagem também contém a palavra como uma cadeia só. No exemplo, o comando NOVO_ENCONTRO é uma cadeia só. Igualmente acontece no modelo da linguagem.

4. Comando-inteiro/Fonemas (Whole command/Phonemes): como na abordagem anterior, o dicionário contém os comandos como cadeias de palavras, mas estas são decompostas em fonemas. O modelo da linguagem é igual ao da abordagem anterior. Neste caso, o comando NOVO_ENCONTRO é decomposto como $n$ o $v$ u e $\sim k$ o $~ t r u$.

\subsection{Projeto e desenvolvimento do software}

A fase de projeto de desenvolvimento do sistema foi constituída por três etapas: a migração do sistema ASR para a plataforma Symbian, a integração com o sistema Borboleta e o desenvolvimento do módulo de interface multimodal para o navegação no sistema usando a voz. Uma descrição mais detalhada dessas etapas é apresentada a seguir.

\subsubsection{Migração do Pocketsphinx}

Com o intuito de executar o Pocketsphinx nos dispositivos Symbian, ele deve ser configurado e/ou adaptado para tal plataforma. Esse processo é chamado normalmente de portar ou migrar e é composto por um conjunto de tarefas que cobre todos os aspectos de modificação 
de código, compilação, configuração e execução. O processo de migração de Pocketsphinx foi baseado no livro de Mark Wilcox, Porting to the Symbian Platform [67] e na comunidade do fórum Nokia ${ }^{1}$. Wilcox propõe um conjunto de passos para obter uma migração satisfatória. Estes passos incluem: seleção do software, análise do código, reestruturação do projeto, configuração de ambiente de desenvolvimento, integração com o sistema de compilação de Symbian, solução de problemas, execução e testes. Cada um desses passos é descrito na Seção C.3.2.

Tendo selecionado o Pocketsphinx como software para ser portado, o passo seguinte foi analisar o código. Essa fase incluiu um estudo da estrutura geral do projeto e definição das dependências de bibliotecas externas. Depois, o ambiente de desenvolvimento para a plataforma Symbian foi configurado (ver descrição no Apêndice G). Na fase de reestruturação e migração do sistema, foram avaliados os locais onde o código deveria ser modificado para suportar a nova plataforma. Dado que uma prévia configuração para Symbian estava incluída no código, ela foi tomada como base para realizar as modificações necessárias. Ao final, 15 arquivos, entre código e arquivos de configuração, foram modificados ou criados. Na integração com o sistema de construção do Symbian foram atualizados os arquivos de configuração de SphinxBase e de Pocketsphinx, e duas configurações adicionais foram criadas para a compilação de dois executáveis de teste do sistema. Uma vez que o Pocketsphinx foi compilado completamente, o passo seguinte foi realizar a configuração dos recursos de memória destinados ao aplicativo, assim como definiram-se os parâmetros de inicialização do decodificador baseados no guia de optimização no site do CMUSphinx ${ }^{2}$. O Pocketsphinx foi testado em dois cenários: no emulador e no dispositivo. No emulador, para verificar se o fluxo geral funcionava corretamente, reduzindo o tempo de implantação no dispositivo real para testes. No dispositivo, foram testados os precedimento de acesso ao hardware, como a inicialização e calibração do microfone. Durante o processo de migração, obteve-se assessoria dos autores do Pocketsphinx, permitindo que ao final da migração as modificações fossem integradas na versão oficial do Pocketsphinx e Sphinxbase.

\subsubsection{Serviço de reconhecimento de fala para dispositivos móveis}

Uma vez que o sistema de reconhecimento de fala tinha sido portado, a etapa seguinte foi a implementação de um serviço de reconhecimento de fala que pudesse ser acessado a partir do Borboleta. Este serviço de reconhecimento de fala para dispositivos móveis (mobile speech recognition service - MSRS) é composto por um módulo de decodificação que acessa o Pocketsphinx, uma interface gráfica para configuração e testes, e um protocolo de comunicação entre aplicações para acessar os serviços de reconhecimento a partir de aplicativos externos usando Java ME ou C++.

Adicionalmente ao reconhecimento de comandos e a execução embutida, cinco requisitos adicionais foram estabelecidos durante o projeto do sistema. Primeiro, o sistema devia ser executado no ambiente Symbian, dado que a maioria dos dispositivos do projeto Borboleta são baseados nessa plataforma. Segundo, a API do Pocketsphinx devia ser acessível com o intuito de disponibilizar o serviço de reconhecimento para outros aplicativos. Terceiro, o sistema devia fornecer uma interface gráfica para a sua configuração básica. Quarto, o sistema devia fornecer um sistema de interação entre aplicativos no dispositivo móvel, tanto para Java ME quanto para C++. Quinto, um módulo Java para interagir com o sistema de reconhecimento de fala devia ser desenvolvido e integrado ao sistema Borboleta.

Baseados nesses requisitos, o sistema de reconhecimento desenvolvido (SpeechMe) foi dividido em dois subsistemas: um módulo em $\mathrm{C}++$ que disponibiliza o serviço de reconhecimento de fala e um módulo em Java que usa esses serviços através de uma conexão por

\footnotetext{
${ }^{1}$ http://www.forum.nokia.com.

${ }^{2}$ http://cmusphinx.sourceforge.net/wiki/pocketsphinxhandhelds.
} 
soquete. O módulo C++ é uma aplicação Qt composta por uma interface gráfica, o decodificador Pocketsphinx, uma camada em $\mathrm{C}++$ que acessa a API do Pocketsphinx e executa o processo de reconhecimento de fala e um submódulo de comunicações baseado em Qt para interagir com aplicações externas. O módulo Java foi integrado com o subsistema multimodal no sistema Borboleta. O módulo Java coordena a comunicação com o módulo em $\mathrm{C}++$ para acessar o serviço de reconhecimento de fala e processa os estados da decodificação para serem apresentados ao usuário.

A Figura 3.2 apresenta a arquitetura geral do sistema com a divisão dos módulos C++ e Java. Na arquitetura, o conceito de formulário multimodal é introduzido. Um formulário multimodal é um formulário normal da camada de apresenta ção, mas que suporta modalidades de entrada adicionais, tais como reconhecimento de fala. No Borboleta, o formulário inclui um botão de ativação que inicia o processo de reconhecimento de fala. Quando o botão é clicado, o serviço de reconhecimento é solicitado ao módulo SpeechMe de Java, o qual redireciona a solicitação ao módulo SpeechRemote de C++ através de um soquete. Já no módulo $\mathrm{C}++$, a requisição é processada chamando o MSRS para iniciar o reconhecimento. Uma vez que o reconhecimento é completado, a resposta é enviada usando o mesmo mecanismo de comunicação. Durante o processo de reconhecimento, mensagens de atualização do estado são enviadas ao módulo Java para serem apresentadas ao usuário. Quando a frase é reconhecida, ela é processada para obter o comando do usuário e ser executado. O botão de ativação para reconhecimento de fala foi utilizado anteriormente por Zhang et al. [71]. A distribuição da aplicação sobre a plataforma Symbian é apresentada na Seção C.3.3. As Seções C.3.4, C.3.5, C.3.6 e C.3.7 apresentam amplamente o desenvolvimento do projeto e a implementação dos módulos em C++ e Java, o esquema de interação e estrutura do sistema de navegação, incluindo definições básicas, diagramas de classe, de sequência e definição do protocolo de comunicação.

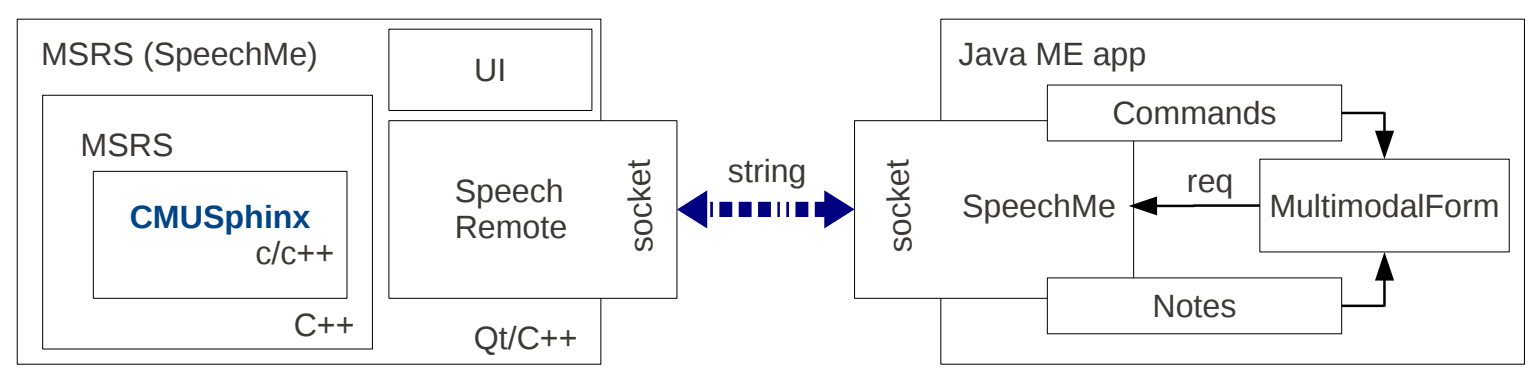

Figura 3.2: Serviço de reconhecimento de fala - Arquitetura geral

\section{Módulo $\mathrm{C}++$}

A interface gráfica do módulo $\mathrm{C}++$ é composta por três $Q$ Widgets: configuração (Figura 3.3.a), interface de teste local (Figura 3.3.b) e busca Web (Figura 3.3.c). Na interface de configuração o decodificador pode ser inicializado usando o arquivo padrão de configuração do Pocketsphinx, onde os parâmetros e endereços dos modelos acústicos e de linguagem são estabelecidos. Também nesta interface é iniciado o servidor de sockets para receber requisições externas (por exemplo, a partir do Borboleta).

A interface de teste local (SpeechPad) é um QWidget que permite testar o reconhecimento de fala sem necessidade de um aplicativo cliente externo. Há duas opções de teste, o reconhecimento isolado, no qual a voz é capturada até o primeiro intervalo de silêncio, e o reconhecimento contínuo, onde a voz é capturada e processada continuamente até o usuário deter o processo.

Uma interface de busca Web usando a voz (SpeechWeb) foi desenvolvida com o intuito de apresentar outras opções de aplicação do reconhecedor de fala no dispositivo móvel. A 
interface posui um botão de ativação para iniciar o reconhecimento de fala; uma vez que a frase é reconhecida, é realizada uma busca no Google e são apresentados os resultados ao usuário usando QWebKit.

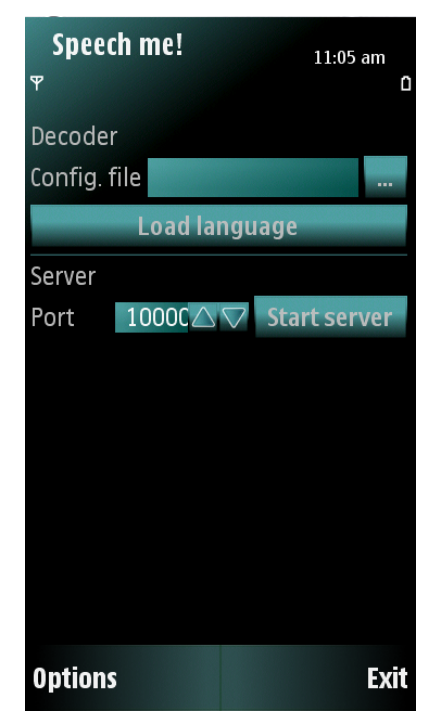

(a) Configuração Speech me!

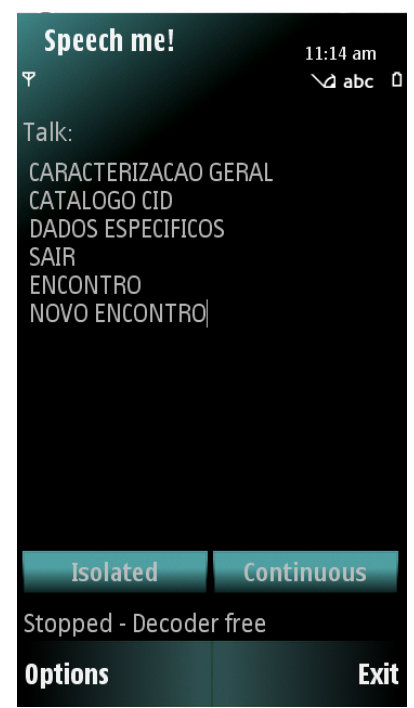

(b) SpeechPad

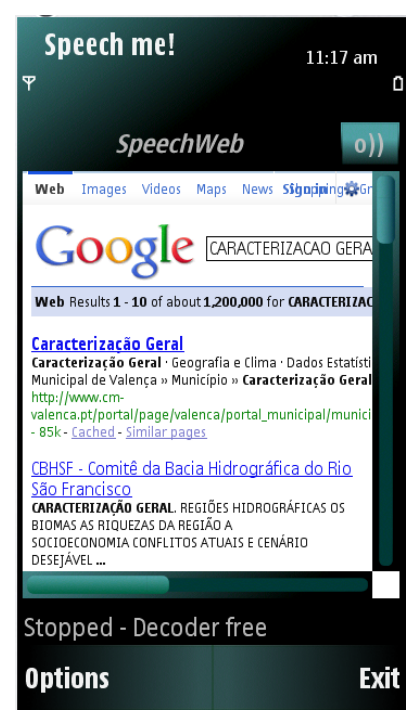

(c) SpeechWeb

Figura 3.3: Interface gráfica do módulo $Q t / C++$ do SpeechMe

\section{Módulo Java ME}

O módulo Java ME é responsável pela escuta de requisições de reconhecimento de fala, o envio destas ao módulo $\mathrm{C}++$ através do socket e o processamento das respostas do decodificador, sejam mensagens de estado ou frases decodificadas. O módulo foi projetado usando o padrão Modelo/Visão/Controlador (MVC) [22], onde o modelo é a classe SpeechMe que controla a comunicação com o módulo $\mathrm{C}++$ e implementa o protocolo de comunicação para envio de requisições e processamento de respostas. A visão é o conjunto de interfaces gráficas que informa ao usuário sobre a disponibilidade do serviço de reconhecimento, a ativação do serviço (Speech button - Figura 3.4) e os quadros de diálogo que notificam o usuário sobre o estado da decodificação (Figura 3.5). O controlador (SpeechController) captura os eventos de reconhecimento e os reenvia à classe SpeechMe. O controlador também recebe as mensagens decodificadas para estabelecer o comando solicitado pelo usuário. Uma vez que o comando é definido, este é executado para realizar a navegação no sistema e apresentar a tela solicitada pelo usuário.

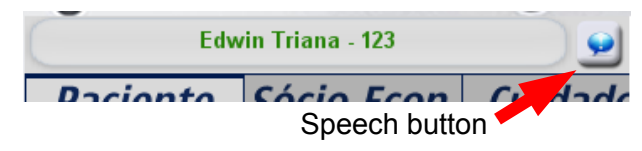

Figura 3.4: Botão de ativação de reconhecimento - SpeechButton

O código-fonte, o corpus de comandos e o programa de coleta de audio estão disponíveis a partir do site do projeto Borboleta ${ }^{3}$.

\footnotetext{
${ }^{3}$ http://ccsl.ime.usp.br/borboleta/pt-br/speech-recognition-0
} 


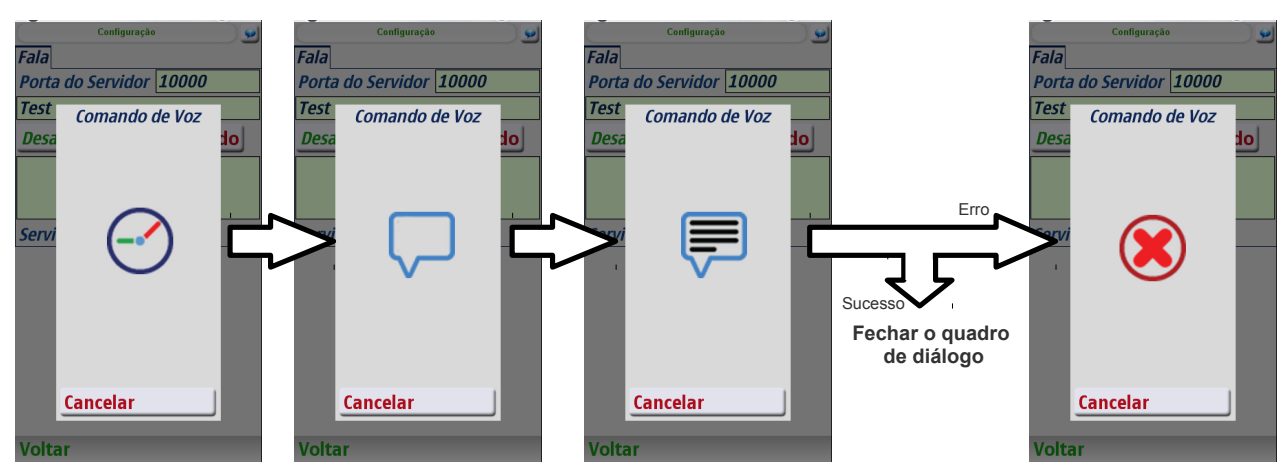

Figura 3.5: Quadro de diálogo durante reconhecimento de comando 


\section{Capítulo 4}

\section{Avaliação}

Três aspectos do sistema foram avaliados: acurácia no reconhecimento de fala, desempenho do sistema e usabilidade. Este capítulo apresenta brevemente a metodologia de avaliação utilizada para os diferentes aspectos e os resultados obtidos. A descrição completa da metodologia usada durante a fase de avaliação é apresentada no Apêndice D.

\subsection{Acurácia no reconhecimento de fala}

Tipicamente, os sistemas ASR são avaliados usando a taxa de erro por palavra (word error rate - WER). Essa taxa mede a precisão do sistema contrastando os sinais reconhecidos com as transcrições verdadeiras. Dois tipos de experimentos foram realizados para avaliar o modelos. O primeiro, fazendo testes automáticos onde $30 \%$ das gravações coletadas foram usadas como sinais para serem processados pelo Pocketsphinx. O segundo foi a experimentação em um ambiente real com as pessoas da equipe do centro de saúde. Antes de este teste no cenário real, um teste de acurácia foi realizado no laboratório para avaliar a eficácia dos modelos antes de serem avaliados com o pessoal do centro de saúde. Também foi utilizada a taxa de erro por sentença (sentence erro rate - SER), que mede o erro do sistema na frase completa, e não em palavras individuais, o qual é importante na avaliação de comandos compostos.

\subsubsection{Descrição dos experimentos}

Ao todo, 16 experimentos foram levados a cabo no para avaliar as possíveis combinações das abordagens propostas no modelamento acústico e de linguagem. Essa quantidade de experimentos foi determinado pelas quatro abordagens para reconhecimento, pelos dois quantidades de estados possíveis ( 3 ou 5 ) e permitindo ou não a omissão de estados. A Tabela 4.1 apresenta a configuração para os 16 experimentos.

Tabela 4.1: Experimentos de acurácia - Testes automáticos

\begin{tabular}{|c|c|c|c|c|}
\hline Estados & Abordagem 1 & Abordagem 2 & Abordagem 3 & Abordagem 4 \\
\cline { 2 - 5 }$/$ Pular & SER / WER & SER / WER & SER / WER & SER / WER \\
\hline $3 /$ no & exp. 1 & exp. 2 & exp. 3 & exp. 4 \\
\hline $5 /$ no & exp. 5 & exp. 6 & exp. 7 & exp. 8 \\
\hline $3 /$ yes & exp. 9 & exp. 10 & exp. 11 & exp. 12 \\
\hline $5 /$ yes & exp. 13 & exp. 14 & exp. 15 & exp. 16 \\
\hline
\end{tabular}

Três ambientes de teste foram configurados. O primeiro, o computador para treinamento, foi compilado e instalado o Sphinxtrain para a criação dos modelos acústicos e o Pocketsphinx para a execução dos testes de acurácia dos 16 experimentos. O segundo cenário foi o emulador de Symbian onde foi instalado o Pocketsphinx, aqui eram executados os mesmos testes que 
os do cenário 1 para a verificação dos resultados. O último cenário foi o teste no dispositivo real, primeiro no laboratório, onde os 5 modelos com melhores resultados de acurácia dos 16 foram testados, e finalmente com a equipe do centro de saúde, onde foi testado o modelo que apresentou o melhor resultado durante os testes no laboratório.

\subsubsection{Resultados de acurácia}

A Tabela 4.2 apresenta as SERs e WERs para os 16 experimentos realizados. Os cinco experimentos com as taxas de erro menores (em negrito) foram usados para fazer os testes no laboratório (resultados na Tabela 4.3). Os resultados do cenário real são apresentados na seção de avaliação de usabilidade.

Tabela 4.2: Resultados de acurácia - Testes automáticos

\begin{tabular}{|c|c|c|c|c|}
\hline $\begin{array}{c}\text { Estados } \\
\text { /Pular }\end{array}$ & Abordagem 1 & Abordagem 2 & Abordagem 3 & Abordagem 4 \\
\cline { 2 - 5 } SER / WER & SER / WER & SER / WER & SER / WER \\
\hline 3 não & ${ }^{1} 22.5 / 17.0$ & ${ }^{2} 10 / 10$ & ${ }^{3} 17.5 / 14.5$ & ${ }^{4} 19.2 / 20.5$ \\
\hline $5 /$ não & ${ }^{5} \mathbf{9 . 2} / \mathbf{6 . 0}$ & ${ }^{6} \mathbf{4 . 2} / \mathbf{4 . 5}$ & ${ }^{7} 8.3 / 8.5$ & ${ }^{8} 9.2 / 11.0$ \\
\hline $3 / \operatorname{sim}$ & ${ }^{9} 21.7 / 14.0$ & ${ }^{10} 25.8 / 26$ & ${ }^{11} 17.5 / 13.0$ & ${ }^{12} 35.8 / 39$ \\
\hline 5 / sim & ${ }^{13} \mathbf{8 . 3} / \mathbf{6 . 0}$ & ${ }^{14} \mathbf{5 . 8} / \mathbf{5 . 0}$ & ${ }^{15} \mathbf{8 . 3} / \mathbf{6 . 0}$ & ${ }^{16} 14.2 / 15.6$ \\
\hline
\end{tabular}

Tabela 4.3: Resultados de acurácia - Laboratório

\begin{tabular}{|c|c|c|}
\hline Experimento & SER(\%) & WER(\%) \\
\hline 5 & 17.3 & 22.2 \\
\hline $\mathbf{6}$ & $\mathbf{4 . 0}$ & $\mathbf{3 . 6}$ \\
\hline 13 & 18.7 & 23 \\
\hline 14 & 10.1 & 8.3 \\
\hline 15 & 20 & 23.3 \\
\hline
\end{tabular}

Baseados nos resultados apresentados nesta seção, para reconhecimento de fala para comandos, usando palavras separadas, com uma linguagem FSG (Finite state grammar), usando fonemas para a representação de palavras com cinco estados e sem permitir omissão de estados (não pular), os modelos acústicos apresentam os melhores resultados. Para este projeto, o experimento 6 tem todas estas características e apresentou as menores taxas de erro.

\subsection{Desempenho do sistema}

Para o desempenho do sistema, foram avaliados o tempo de resposta para o reconhecimento e execução dos comandos e o uso de recursos (Memória e CPU). Para coletar os dados de desempenho nos dispositivos móveis foi usado o Nokia Energy Profiler.

O experimento foi levado a cabo no dispositivo Symbian (Nokia N97, OS v9.4 S60, $434 \mathrm{MHz}$ ARM 11 processor, 128MB RAM) da seguinte forma: uma vez inicializado o sistema Borboleta, o profiler foi executado e o monitoramento foi iniciado. Depois, uma tarefa foi executada a cada trinta segundos para identificar as mudanças nos dados coletados. As tarefas executadas foram as seguintes:

1. Iniciar SpeechMe.

2. Iniciar o decodificador (carregar os modelos acústicos e de linguagem).

3. Iniciar o reconhecimento isolado (SpeechPad). 
4. Iniciar o servidor de soquetes.

5. Ativar o reconhecimento de fala no Borboleta.

6. Iniciar o reconhecimento de comandos no Borboleta.

7. Desativar o reconhecimento no Borboleta e fechar o SpeechMe (liberação de recursos).

Como é apresentado na Figura 4.1, O SpeechMe reserva 5\% (6,4Mb) da memória disponível durante a inicialização. Durante a busca do arquivo de configuração, o seletor de arquivos de Qt reserva um $4 \%$ adicional $(3,84 \mathrm{Mb})$, depois a média de memória utilizada foi $7,6 \mathrm{Mb}$ $(6 \%)$. O pico entre as tarefas 4 e 5 foi atribuído à instanciação do formulário de configuração de reconhecimento de fala. No final da execução do teste, todos os recursos são liberados completamente. A figura também apresenta o uso da CPU durante o teste. A inicialização (Tarefa 1) foi rápida e não usou muito tempo de CPU. Os picos entre as tarefas 1 e 2 foram devido à busca do arquivo de configuração. O uso de CPU na tarefa 2 , à configuração e inicialização do decodificador. Os picos entre as tarefas 2 e 3 foram gerados pela troca de widget entre o Config e SpeechPad. O reconhecimento isolado (tarefa 3) usa a CPU duas vezes, primeiro quando o microfone é aberto e a captura de sinais é iniciada, e segundo, durante o processamento do sinal e a decodificação. Picos gerados entre tarefas 3 e 4 novamente foram atribuídos à troca de widget do SpeechPad para o ConfigUi para a inicialização do servidor de soquetes (tarefa 4), a qual usa pouco tempo de CPU. A troca de aplicação do SpeechMe para o Borboleta ocasionou o uso de CPU entre as tarefas 4 e 5 . A conexão ao soquete foi rápida e a maioria do tempo de CPU é utilizado na requisição de permissão de acesso a esse serviço ao usuário. O reconhecimento de comandos (tarefa 6) inclui a apresentação do quadro de estado (SpeechDialog), a requisição do serviço de reconhecimento, a atualização de estados no quadro de diálogo, o processamento do resultado e a execução do comando para a troca de tela no Borboleta. Fechar as conexões e finalizar o Speech (tarefa 7) usou um tempo de CPU normal. Também com o Nokia Profiler, o nível de voltagem da bateria foi monitorado durante os testes. Ele apresentou uma leve variação de $4.02 \mathrm{~V}$ para $3.98 \mathrm{~V}$, tendo em conta que esta redução incluiu a inicialização do decodificador e o reconhecimento de fala. No uso normal, o decodificador só e inicializado uma vez. Assim pode-se concluir que a tempo de vida da bateria do celular não é fortemente afetado pela execução do decodificador e a comunicação via soquetes. Em geral, o desempenho do sistema encontra-se dentro dos limites das aplicações comuns. Não existem laçoes em espera ativa, nem tarefas extras de processamento durante a execução do SpeechMe.

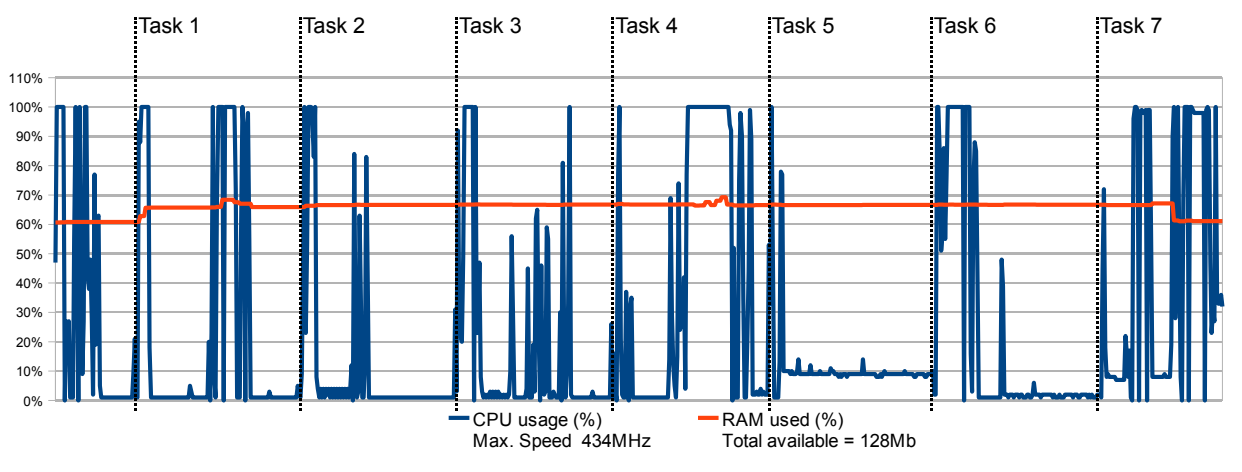

Figura 4.1: Uso de memória e $C P U$ 


\subsection{Usabilidade do sistema - Caso de estudo Borboleta}

Esta seção descreve a metodologia proposta para a avaliação da usabilidade do esquema de navegação usando reconhecimento de fala para o sistema Borboleta.

A avaliação envolveu duas fases: o teste de usabilidade inicial sem funcionalidades de reconhecimento de fala (não multimodal) e o teste de usabilidade depois da implementação do serviço (multimodal). O objetivo foi avaliar se o uso de reconhecimento de fala melhora a experiência do usuário durante o uso do sistema. O público alvo foi os usuários do sistema no centro de saúde. Cada usuário executou cinco tarefas de navegação. Cada tarefa é uma dupla de uma tela de início e uma tela alvo. A definição das tarefas foi baseada no esquema de navegação do sistema (ver Figura 3.1). A Tabela 4.4 sumariza as tarefas selecionadas, indicando a tela inicial, a tela final, a função exigida no caso de telas multifuncionais e o custo ou comprimento do caminho, isto é, a quantidade de cliques para chegar à tela final.

Ao final do experimento, os usuários avaliaram a sua experiência usando o sistema por meio de um questionário de usabilidade baseado no mobile phone usability questionnaire (MPUQ), envolvendo 6 fatores: facilidade de uso, capacidade de solução de problemas, propriedade multimídia, carga mínima de memória, controle e eficiência e tarefas típicas para celular. [52].As perguntas foram divididas em duas partes: um conjunto de três perguntas onde as tarefas específicas são avaliadas e outro grupo de cinco perguntas gerais sobre o sistema e o desempenho durante o teste. Cada tarefa contém as seguintes perguntas: (1) A tarefa foi completada? (2) Qual foi o nível de dificuldade para completar a tarefa? e Quantidade de cliques. No grupo geral as perguntas foram: (1) Qual é o nivel de dificuldade para navegar no sistema? (2) O que você acha da estrutura da aplicação? (3) Encontrou as ferramentas onde você achava que estavam? (4) Foi fácil usar menus na tela? e (5) Sentiu-se confortável ao usar a aplicação? Ao final, deixaram-se dois campos abertos para sugestões e comentários. No teste multimodal, a pergunta 4 foi substituída por: Foi fácil usar reconhecimento de fala? O Figura 4.2 apresenta o questionário completo utilizados durante os testes de usabilidade.

\subsubsection{Resultados de usabilidade}

Os testes de usabilidade foram realizados com quatro pessoas (um homem e três mulheres) da equipe do centro de saúde, sendo um médico, uma médica e duas enfermeiras. Nas Tabelas 4.5 e 4.6 são apresentados os dados coletados durante o teste de usabilidade não multimodal. Os resultados do teste de usabilidade com interface multimodal são apresentados nas Tabelas 4.7 e 4.8. O tempo de execução de cada tarefa foi medido manualmente a partir do momento em que o usuario clicava no botão de ativação de fala até quando a nova janela era apresentada. As taxas de erro para o modelo acústico utilizado (experimento 6 da tabela 4.2 ) foi de $5 \%$ para SER e $4.2 \%$ para WER.

Tendo em vista os resultados coletados durante os testes de usabilidade, uma melhora geral nos níveis de usabilidade foi observada. Embora em ambos os testes as tarefas foram completadas, o tempo médio para completar uma tarefa foi reduzido na maioria das tarefas. Para tarefas sem telas de seleção intermediárias, o tempo de conclusão foi reduzido de um

Tabela 4.4: Lista de tarefas - teste de usabilidade

\begin{tabular}{|c|c|c|c|c|}
\hline Tarefa & Tela inicial & Tela final & Função & Custo \\
\hline 1 & Dados específicos & Agendar visita & - & 5 \\
\hline 2 & Caracterização geral & Encontros realizados & - & 6 \\
\hline 3 & Adicionar diagnóstico & Paciente & - & 5 \\
\hline 4 & Adicionar diagnóstico CID & Encontro domiciliar & Encontro antigo & 8 \\
\hline 5 & Agenda & Encontro & novo encontro & 3 \\
\hline
\end{tabular}


Projeto Borboleta

Questionário de usabilidade - Navegação

Não multimodal

Nome:

\section{Perguntas específicas}

1 Dados específicos - Agendar visita

1 A tarefa foi finalizada?

2 Qual foi o nível de dificuldade para completar a tarefa?

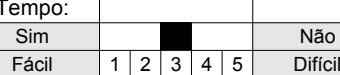

3 Número de cliques

2 Caracterização geral - Encontros realizados

1 A tarefa foi finalizada?

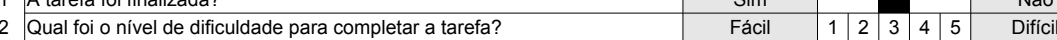

3 Número de cliques

3 Adicionar diagnostico - Paciente

1 A tarefa foi finalizada?

2 Qual foio nivel de dificuldade para completar a tarefa?

Sim $\quad$ Não

3 Número de cliques

4 Adicionar diagnostico de IDC - Encontro (antigo)

1 A tarefa foi finalizada?

2 Qual foi o nível de dificuldade para completar a tarefa?

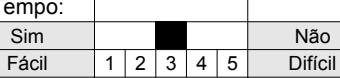

3 Número de cliques

5 Agenda - Novo encontro

1 A tarefa foi finalizada?

2 Qual foi o nível de dificuldade para completar a tarefa?

Tempo:

Número de cliques

\section{Perguntas gerais}

1 Qual é o nível de dificuldade para navegar no sistema?

2 O que você acha da estrutura da aplicação

Encontrou as ferramentas onde você achava que estavam?

Foi fácil usar menus na tela

Sentiu-se confortável ao usar a aplicação?

\begin{tabular}{l|lll|ll|l} 
Fácil & 1 & 2 & 3 & 4 & 5 & Difícil
\end{tabular}

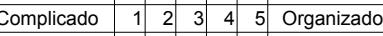
\begin{tabular}{l|rrrrrr} 
Nunca & 1 & 2 & 3 & 4 & 5 & Sempre \\
\hline
\end{tabular}

Comentários:

Sugestões:

Figura 4.2: Questionário de usabilidade - não multimodal 
Tabela 4.5: Resultados de teste de usabilidade - Perguntas específicas - Não multimodal

\begin{tabular}{|c|c|c|c|c|c|c|c|c|c|}
\hline \multirow[b]{3}{*}{ Tarefas } & \multicolumn{8}{|c|}{ Perguntas } & \multirow[b]{3}{*}{ Tempo $^{\S}$} \\
\hline & \multicolumn{2}{|c|}{$1^{*}$} & \multicolumn{5}{|c|}{$2^{*}$} & $3^{+}$ & \\
\hline & Sim & Não & 1 & 2 & 3 & 4 & 5 & Cliques & \\
\hline 1 & 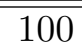 & & & 25 & 75 & & & $\overline{77}$ & 8.3 \\
\hline 2 & 100 & & & 75 & 25 & & & $7.5 \approx 8$ & 11 \\
\hline 3 & 100 & & & 50 & 50 & & & $6.5 \approx 7$ & 11.8 \\
\hline 4 & 100 & & & 25 & 75 & & & $8.3 \approx 8$ & 12 \\
\hline 5 & 100 & & 75 & 25 & & & & $3.8 \approx 4$ & 7.3 \\
\hline
\end{tabular}

* Porcentagens

+ Número de cliques

$\S$ Média em segundos

Tabela 4.6: Resultados de teste de usabilidade - Perguntas gerais - Não multimodal

\begin{tabular}{|l|c|c|c|c|c|}
\hline \multicolumn{1}{|c|}{ Pergunta } & \multicolumn{5}{c|}{$\%$} \\
\cline { 2 - 7 } & 1 & 2 & 3 & 4 & 5 \\
\hline \hline 1 Nível de dificuldade na navegação (baixo é bom) & & 25 & 75 & & \\
\hline 2 Estrutura da aplicação (alto é bom) & & & & 100 & \\
\hline 3 Ferramentas no lugar certo (alto é bom) & & & 25 & 75 & \\
\hline & \multicolumn{2}{|c|}{ Sim } & \multicolumn{3}{c|}{ Não } \\
\hline 4 GUI fácil de usar & \multicolumn{2}{|c|}{25} & \multicolumn{2}{c|}{75} \\
\hline 5 Confortável no uso da aplicação & \multicolumn{1}{|c|}{100} & \multicolumn{2}{|c|}{} \\
\hline
\end{tabular}

Tabela 4.7: Resultados de teste de usabilidade - Perguntas específicas - Multimodal

\begin{tabular}{|c|c|c|c|c|c|c|c|c|}
\hline \multirow[b]{3}{*}{ Tarefas } & \multicolumn{7}{|c|}{ Perguntas } & \multirow[b]{3}{*}{ Tempo $^{\S}$} \\
\hline & \multicolumn{2}{|c|}{$1^{*}$} & \multicolumn{4}{|c|}{$2^{*}$} & \multirow{2}{*}{$\begin{array}{c}3^{+} \\
\text {Cliques }\end{array}$} & \\
\hline & Sim & Não & 1 & 2 & 3 & 4 & & \\
\hline 1 & 100 & & 100 & & & & 1 & 5.3 \\
\hline 2 & 100 & & 100 & & & & 1 & 5.5 \\
\hline 3 & 100 & & 100 & & & & 1 & 5 \\
\hline 4 & 100 & & 100 & & & & 2 & 8.3 \\
\hline 5 & 100 & & 100 & & & & 2 & 7.3 \\
\hline
\end{tabular}

* Porcentagens

+ Número de cliques

$\S$ Média em segundos

Tabela 4.8: Resultados de teste de usabilidade - Perguntas gerais - Multimodal

\begin{tabular}{|l|c|c|c|c|c|}
\hline \multicolumn{1}{|c|}{ Perguntas } & \multicolumn{5}{c|}{ \% } \\
\cline { 2 - 6 } & 1 & 2 & 3 & 4 & 5 \\
\hline \hline 1 Nível de dificuldade na navegação (baixo é bom) & 100 & & & & \\
\hline 2 Estrutura da aplicação (alto é bom) & & & & 100 & \\
\hline 3 Ferramentas no lugar certo (alto é bom) & & & & 75 & 25 \\
\hline & \multicolumn{2}{|c|}{ Sim } & \multicolumn{4}{|c|}{ Não } \\
\hline 4 Reconhecimento de fala fácil de usar & \multicolumn{2}{|c|}{100} & \multicolumn{3}{|c|}{} \\
\hline 5 Confortável no uso da aplicação & 100 & \multicolumn{4}{|}{} \\
\hline
\end{tabular}


máximo de 11,8 segundos para um intervalo de cinco a seis segundos. As tarefas que exigem retroalimentação do usuário foram mais demoradas devido à interação normal com o usuário (Figura 4.3). A segunda tarefa, que tinha o menor número de cliques, apresentou um incremento leve no tempo de execução. Para tarefas com nível de dificuldade relativamente baixo (1 ou 2) no teste não multimodal, este nível de dificuldade foi reduzido para 1 no teste multimodal (Figura 4.4). O resultado mais importante no grupo de perguntas específicas foi a redução do número de cliques para 1 (ativação do serviço de reconhecimento), em tarefas onde não é necessária a intervenção do usuário, e para dois (ativação do serviço de reconhecimento mais interação adicional do usuario), em tarefas onde uma tela intermediária é apresentada para o usuário para coletar informação para completar a tarefa. Essa redução resultou em um melhoramento na usabilidade e na redução do nível de atenção exigida para completar uma tarefa.

Os indivíduos afirmaram que a navegação através do sistema tornou-se mais fácil usando reconhecimento de fala. A percepção da estrutura da aplicação foi melhorada levemente, assim como a organização das funcionalidades (Figura 4.5). Os comentários da equipe de saúde sugerem que o uso de reconhecimento de fala como entrada do sistema foi bem aceito devido à redução de tempo e à acurácia do sistema. Os usuários também sugeriram incluir mais comandos para acessar outras funcionalidades do sistema e aumentar o tamanho dos menus para evitar ativar elementos adjacentes. Em geral, os usuários avaliaram como boa a localização do botão de ativação de reconhecimento de fala (canto superior-direito), mas apresentaram uma sugestão de colocar o botão na parte inferior-central da aplicação. Depois de explicar as convenções do processo de reconhecimento, os usuários identificaram facilmente o momento quando podiam ditar o comando e quando o decodificador estava coletando e processando os sinais.
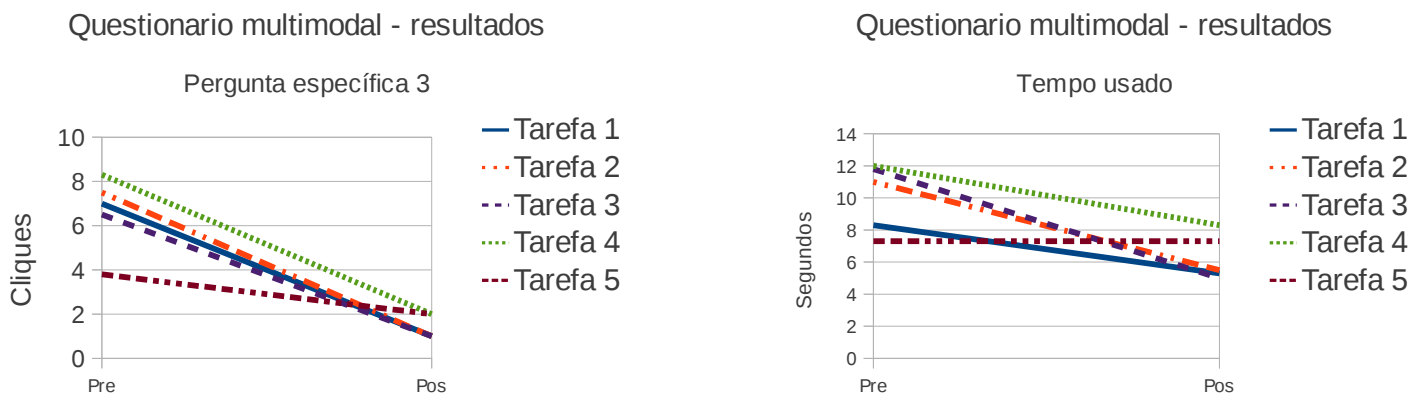

Figura 4.3: Redução de cliques e tempo

Questionario não multimodal - resultados

Pergunta específica 2

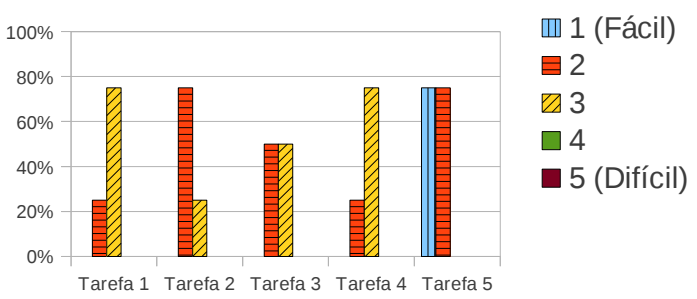

Questionario multimodal - resultados

Pergunta especifica 2

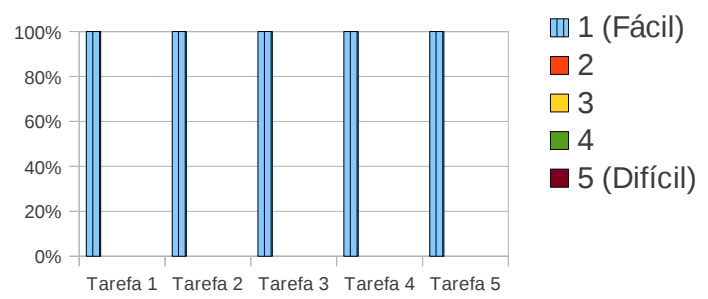

Figura 4.4: Resultados de perguntas específicas 
Questionario não multimodal - results

Perguntas gerais (Escala Likert)

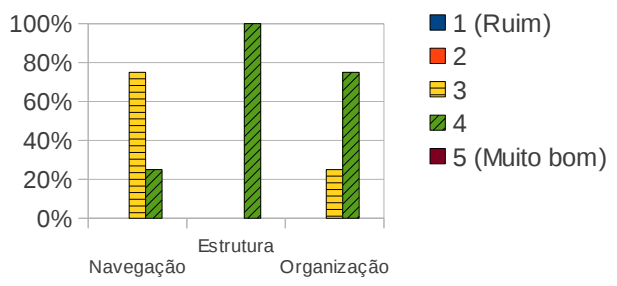

Questionario multimodal - resultados

Perguntas gerais (Escala Likert)

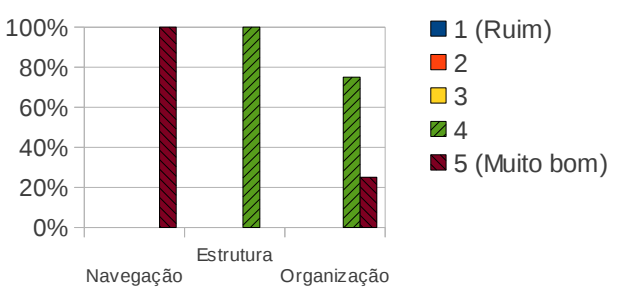

Figura 4.5: Resultados de perguntas gerais 


\section{Capítulo 5}

\section{Discussão}

Este capítulo aborda a questão de como este projeto contribuiu no trabalho de interfaces multimodais em dispositivos móveis, se os objetivos foram alcançados e quais poderiam ser os trabalhos futuros nesta área. As conclusões deste projeto são apresentadas e contrastadas com os objetivos definidos no começo do projeto. Oportunidades de pequisa e aprofundamento são apresentadas no final do capítulo.

Usar dispositivos móveis na vida cotidiana tem se tornado normal nas cidades e, em um nível menor, nas áreas rurais. A maioria dos cidadãos, em cidades como São Paulo, têm pelo menos um dispositivo móvel (celular ou PDA). Na última década, esses dispositivos têm evoluído drasticamente. Processadores mais rápidos, mais memória, maior capacidade de armazenamento, mais sensores e as múltiplas opções de conexão têm transformado estes simples aparelhos em pequenas estações de trabalho. No entanto, devido aos requerimentos de portabilidade, esses dispositivos continuam sendo pequenos. Mais componentes gráficos têm sido disponibilizados para o desenvolvimento de aplicações mais elaboradas, mas o uso de interfaces simples segue sendo um requerimento de usabilidade. As modalidades de entrada de dados destes dispositivos não são suficientes para as atuais necessidades de mobilidade. Teclados qwerty embutidos facilitam a entrada de dados nesses dispositivos. No entanto, nem sempre os usuários podem estar com toda sua atenção voltada para a entrada ou saída desses dispositivos. As interfaces multimodais (MMI) têm aberto um novo campo de pesquisa na interação homem/computador.

As MMI permitem o uso dos computadores de um jeito mais natural estendendo os métodos tradicionais de entrada/saída, seja misturando os existentes ou criando novos. Devido à facilidade do uso da voz para se comunicar, os pesquisadores têm abordado o desafio de que os computadores entendam melhor as requisições dos usuários usando a voz, aproveitando os avanços na pesquisa em reconhecimento de fala. O uso de modelos estatísticos e de metodologias de processamento de linguagens tem resultado em altos níveis de reconhecimento de fala isolada e contínua. O uso de reconhecimento de fala em dispositivos móveis traz grandes benefícios para a interação com o usuário, estendendo os limitados modos de entrada para uns mais abrangentes.

Este trabalho apresenta o uso de reconhecimento de fala como método de entrada multimodal a fim de melhorar o uso de um sistema móvel de coleta de dados em visitas domiciliares. Os incrementos nos níveis de usabilidade do sistema Borboleta podem resultar na melhora no atendimento dos pacientes durante os encontros domiciliares devido à redução do nível de atenção exigido na manipulação do sistema. Os objetivos deste projeto foram verificar se o uso do reconhecimento de fala como método de entrada melhora os níveis de usabilidade de uma aplicação com o Borboleta, avaliar se os dispositivos móveis atuais estão preparados para a execução de tarefas complexas como o processamento e decodificação de sinais em tempos de resposta aceptáveis, sem afetar o desempenho do dispositivo, e contribuir para a pesquisa em reconhecimento de fala para português brasileiro. 
Esta dissertação apresentou uma revisão dos conceitos básicos de interfaces multimodais, reconhecimento de fala e plataformas móveis. Também foi apresentada a metodologia e o desenvolvimento do projeto e como um sistema de reconhecimento de fala (Pocketsphinx) foi integrado ao Borboleta sobre a plataforma Symbian. Finalmente, foi apresentado o processo de avaliação dos diferentes aspectos do projeto: o desempenho do software, a acurácia no reconhecimento de comando e a usabilidade do sistema.

Verificou-se que os níveis de reconhecimento de fala para comandos em português brasileiro estão em um intervalo aceitável para aplicações de uso geral. A avaliação de quatro abordagens diferentes para o reconhecimento de comando, permitiu concluir que a representação de palavras por méio de fonemas e o uso de modelos de linguagem baseados em palavras isoladas reduz as taxas de erro no reconhecimento de comandos.

No desempenho do software, os resultados mostraram que os dispositivos móveis atuais estão preparados para a execução de tarefas de reconhecimento para modelos acústicos e de linguagem pequenos, como comandos ou números, com tempos de resposta aceitáveis. Para vocabulários grandes, o reconhecimento deveria ser implementado através das outras duas abordagens: reconhecimento distribuído, onde os vetores de observação são obtidos no aparelho e a decodificação é feita em um servidor distante; ou reconhecimento em rede, onde todo o processo de reconhecimento é realizado em um servidor distante. Também foi verificado que a integração de aplicações em $\mathrm{C}++$ e Java usando soquetes é uma solução viável para o acesso de funções externas a partir de Java, sem afetar o desempenho do dispositivo.

$\mathrm{Na}$ área da usabilidade, esta pesquisa contribuiu para o projeto Borboleta no trabalho em dispositivos móveis estendendo o projeto para a inclusão de interfaces multimodais. $\mathrm{O}$ uso de reconhecimento de fala como método alternativo de entrada deu ao usuário a opção de interagir com a aplicação de uma forma mais natural. A experiência do usuário foi melhorada, especialmente para usuários principiantes que não tinham familiaridade com o sistema. Devido às baixas taxas de erro, os usuários aceitaram facilmente a opção de reconhecimento de fala e, em vários casos, foi a opção preferida. Os usuários sugeriram a extensão do vocabulário para manipular a aplicação. A interface multimodal foi amplamente aceita devido às altas taxas de reconhecimento, ao fácil acesso ao botão de ativação do serviço, à clara retroalimentação ao usuário sobre o processo de decodificação e à importante redução do tempo na manipulação do sistema.

Entre outras contribuições encontram-se a inclusão das modificações do Pocketsphinx na linha principal de desenvolvimento do projeto disponível no site do CMUSphinx ${ }^{1}$, o interesse da Nokia (Brasil) na colaboração com o desenvolvimento do projeto SpeechMe, o desenvolvimento de uma aplicação para a coleta de amostras de áudio, a construção de um corpora básico de comandos para português brasileiro e a possibilidade de aproximar os avanços em reconhecimento de fala à sociedade através de novos paradigmas de interação.

O código fonte do SpeechMe $(\mathrm{C}++/ \mathrm{Qt})$ encontra-se no repositório público da Nokia ${ }^{2}$, sob licença BSD. O módulo Java encontra-se no repositório do IME-USP ${ }^{3}$. Os modelos gerados e os arquivos de áudio e transcrições foram disponibilizados no site do Borboleta ${ }^{4}$.

\subsection{Trabalhos futuros}

Devido às diferentes áreas que foram abordadas durante esta pesquisa, existem várias oportunidades de pesquisa para estender o presente trabalho. Existem importantes iniciativas no campo do reconhecimento de fala para português brasileiro, como o projeto FalaBrasil, para o desenvolvimento de sistemas robustos para reconhecimento de fala e de modelos

\footnotetext{
${ }^{1}$ http://cmusphinx.sourceforge.net.

${ }^{2}$ https://projects.forum.nokia.com/speechme.

${ }^{3}$ svn://ccsl.ime.usp.br/borboleta

${ }^{4}$ http://ccsl.ime.usp.br/borboleta/pt-br/speech-recognition-0
} 
acústicos e de linguagem de alta qualidade. Nessa linha de pesquisa, pode-se trabalhar no melhoramento de reconhecimento de comandos aumentando o tamanho do vocabulário ou incluindo módulos de processamento de linguagens que permitam ao usuário realizar requisições ao sistema de uma forma mais natural. No campo médico, o reconhecimento de fala contínua poderia ser utilizado na transcrição de notas médicas gravadas durante os encontros domiciliares, evitando que o profissional tenha que inserir dados da forma convencional, o que reduziria o foco na manipulação do dispositivo. Os dispositivos atuais oferecem bons resultados no processamento de fala com vocabulário pequena utilizando abordagens de reconhecimento embutido, no entanto, para vocabulários maiores ainda é necessário o uso de serviços externos.

Outro campo de pesquisa que poderia estender este trabalho é a otimização dos algoritmos de processamento de sinais e busca de palavras para reduzir o tempo de resposta em dispositivos móveis no uso de abordagens de reconhecimento embutido, estendendo o suporte para vocabulários maiores ou aumentando o tempo de vida dos dispositivos. Para abordagens de reconhecimento em rede e distribuído, as estruturas de representação e os protocolos de comunicação poderiam ser estudados a fim de buscar melhorias no uso do canal de comunicação e do tempo de resposta no reconhecimento. 


\section{Parte II}

\section{Apêndices \\ Relatório técnico (Inglês)}


Brazilian Portuguese Speech Recognition for Navigation on Mobile Device Applications

Master student: Edwin Miguel Triana Gómez

Advisors: Fabio Kon and Rebecca Bates 


\section{Appendix A}

\section{Introduction}

New technologies on mobile devices have increased their application range. Initially, these were used as mobile phones, note takers, or address directories. New features include high performance processors enabling the use of better graphical user interfaces and execution of specialized tasks, multi-platform, high-level development environments such as Java ME, wireless LAN (Wi-Fi) for faster connectivity, global positioning systems, and multi-touch screens. At the same time, new software has been developed, from small applications like multimedia players to more complex ones like Web browsers or calendar managers. However, the small screens have been a drawback for the use of sophisticated applications. Too many functions on tiny screens or too many window changes to complete a task result in low user acceptance levels [34]. Multi-modal interfaces are one approach to this issue, where input methods such as touch screens, buttons, and voice allow clear interfaces and receive better usability results.

A specialized application is the Borboleta system, a Java ME project to collect medical information in the primary care program of the Brazilian health system SUS [13], being developed at the University of São Paulo FLOSS Competence Center. ${ }^{1}$ Since the Borboleta system offers different application features, good device interaction like well organized forms, shortcuts, menus, feedback (e.g., dialogs or sounds) and speech recognition to execute user commands is important.

One advantage of using voice input is that it is a natural skill and does not require much attention or direct observation to use the device, allowing increased attention to other activities [47]. Other advantages are the reduction of hand use or keyboard interaction to input information. This makes access easier for handicapped persons. Nevertheless, using voice input for interaction has some challenges such as environment noise, speech accent, large vocabularies, low quality microphones, and device capability in voice processing. In the last twenty years, robust speech recognition systems have addressed these problems. Two open source examples are HTK [68] and Sphinx 4 [65], which have very acceptable performance levels with low word error rates [54]. However, these systems were developed for desktop computers with good processing resources. In mobile devices, the limited processing and storage resources make the implementation of voice recognition systems even harder.

This work examines human computer interaction for the Borboleta system and the implementation of an automatic speech recognition (ASR) module to the navigation schema.

\section{A.1 Problem statement}

Mobile devices offer the opportunity of fast access to information, at any moment, in practically any place. That availability motivates researchers and developers to implement

\footnotetext{
${ }^{1}$ http://ccsl.ime.usp.br/borboleta
} 
new applications. The goal in these implementations is to provide useful tools to access information in work environments where normal computers cannot be easily used. Issues such as device size and wired-electricity dependency make desktop computers unfit for many tasks. Mobile devices can better handle non-regular workplaces, such as data collecting in outdoor environments.

In the mobile devices field, useful or important applications require user attention and focus to produce correct input or perceive device output. However, when these are done in combination with other important tasks, there can be detrimental effects to the task at hand as well as to the data input. An example is getting directions from a GPS system while driving in a large city, which requires entering a destination address and reading the result from a 5 inch screen. An approach that tries to reduce this type of problem is the use of multi-modal interfaces [47], involving different input and output methods to reduce the required attention level by making the device easier to use. An example of this approach is the use of speech recognition as an input method along with a touch screen and speech synthesis to indicate system response.

Borboleta is a system for collecting patient information using mobile devices, which requires a high level of user attention. During a medical visit, the doctors see the patients, check their health conditions and at the same time input medical information into the device. While using the Borboleta system, if the professional is focused on system operation, he or she may skip important patient information. On the other hand, if he or she focuses on the patient, he or she may manipulate the device incorrectly and record erroneous information. Moreover, medical visits are time limited due to patient commitments and other scheduled visits for the professional. Data collection procedures must be done quickly, while still collecting accurate and useful patient information.

\section{A.2 Objectives}

Three objectives were defined in the beginning of the project. The first objective was to verify if the introduction of voice as an alternative input method to navigate through the system will improve the Borboleta system's usability. If so, professionals could focus on the more important activity of patient care and less on the application operation. Currently, Borboleta has a sophisticated user interface because of its multiple functionalities. That interface is composed of approximately 23 different screens, which contain different graphical components such as tabs, textfields, lists, and checkboxes, specific system menus and a defined navigation schema. To access any functionality in the system, it is necessary to know the complete path to reach it. Such a path could have more than two steps or intermediate windows, increasing the operation time for an individual task. A possible solution is the use of shortcuts to go to specific places, but this requires adding new interaction elements such as menus or buttons. Including new buttons increases the interaction elements displayed on the small screen.

The second objective was to use a small Portuguese vocabulary to control the Borboleta system, and to open the path for speech recognition of health care notes, requiring a larger specialized vocabulary. This objective was established because speech recognition of the English language has been widely studied in previous work, but Brazilian Portuguese has had few initiatives in this field. There have been recent efforts to provide tools for speech recognition for Brazilian Portuguese, such as the FalaBrasil initiative [44]. Most of these projects are based on a vocabulary of commonly used words. In the medical domain, there are no relevant projects that use Brazilian Portuguese for speech recognition.

The third and final objective was to explore the behavior of different command recognition approaches for navigation in the Borboleta system and establish which approach offers better results. Each possible approach has strengths and weakness depending on the problem 
domain. In order to implement an automatic speech recognition (ASR) system on mobile platforms we evaluated whether the mobile devices currently used in Borboleta can support speech recognition and whether the implementation improves the user experience controlling the mobile device. Previous work has implemented English speech recognition for mobile devices [31, 70], but most of these have been distributed speech recognition, where the signal processing phase happens on the device and the actual speech recognition happens on a distant server. For system navigation, all calculations need to be executed on the mobile device; in the case of the Borboleta project, the mobile devices do not even have network connectivity during home visits.

\section{A.3 Overview}

This part of the document consists of a literature review covering multimodal interfaces, speech recognition, mobile computing and a brief description of the Borboleta system, followed by a project development section, which describes the approach to addressing the question of the usefulness of a multimodal interface to the Borboleta mobile system, and presents the architecture of the proposed system. Next an evaluation section is presented, where speech recognition accuracy results are presented, usability testing results are analyzed and the software performance is evaluated. The document concludes with a discussion of the results and future implications of this work. 


\section{Appendix B}

\section{Background}

The task of speech recognition incorporates concepts from theoretical and applied sciences including signal processing, stochastic processes, search algorithms, and software engineering. This section provides a basic concept overview of multimodal interfaces, followed by a historical review of speech recognition, its state of the art, and the basis of the field. After that, a description of existing Brazilian Portuguese speech corpora is presented. The Borboleta system used in this work is presented, followed by a description of mobile platforms and development environments. The section concludes with a discussion of related work.

\section{B.1 Multimodal interfaces}

A multimodal system processes two or more input methods to respond to a user request. The processing can be combined to create a simple request, solve an ambiguous situation [46], or to provide alternative input modes [60]. Common input methods are keyboards, mouse, pen, touch screens, speech recognition, accelerometers and gestures. Figure B.1 shows the different input/output facilities available on mobile devices. These multimodal inputs are often combined with a multimedia output, such as visual interfaces or speech synthesis. The aim of multimodal interfaces (MMI) is to improve human-computer interaction by emphasizing the use of richer and more natural ways of communication [19]. In contrast to the common interfaces, where the request of a user is easily determined by the input, for example a button clicked or a key pressed, MMI inputs often combine signal streams (audio or video) that must be processed and interpreted with a degree of uncertainty because of probabilistic processes [19].
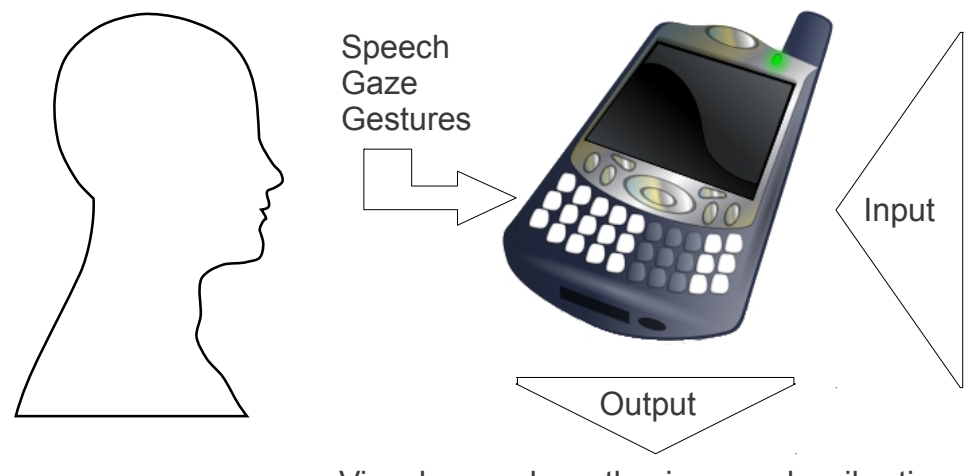

Acceleration

Keyboard

Touchscreen

Pen

Camera

Microphone

GPS

Trackers

Visual, speech systhesis, sounds, vibration

Figure B.1: Input/Output facilities on mobile devices

According to Oviatt [46], there are two input mode types in MMI, the active mode where 
the request or command is given to the computer in an intentional manner, for example a speech command or a gesture on a touch a screen, and the passive mode, where the system captures user behaviors or actions to use as inputs that have not been explicitly expressed. Facial expressions and body movements are examples of passive input modes. In the same way, multimodal interfaces can be classified in different groups. The simplest case is to offer independent alternative input modes (unimodal) to execute a task, giving more options to users. If the input is composed of active and passive input modes, the system uses a blended multimodal interface, for example, using speech and lip movements. The most complex case is the temporally-cascaded multimodal interface. Here the processing of two or more inputs is done sequentially, so current input restricts the interpretation of the following input.

One advantage that MMI brings to systems is the improvement in the interpretation of the user requests by combining different partial information from the input sources. Systems can also be customized or adapted to different users and contexts in a more transparent way. Using MMI is not necessarily more efficient than WIMP (Windows, Icons, Menu, Pointing device) interfaces. In [19], it is stated that there was only $10 \%$ improvement in task completion. More significantly, using MMI, users made $36 \%$ fewer errors, and over $95 \%$ of the users prefered multimodal interaction over unimodal interaction. This error rate reduction could be derived from the lower cognitive work load as described by the short-term memory model of Baddeley and Hitch [2]. This model asserts that a person who performs two simultaneous tasks could be efficient in each one if each of these belongs to a different perceptual domain (e.g., visual and verbal tasks). This is because two of the three main components of short-time memory are dedicated to the auditory-verbal and visuospatial input processing, while the other one acts as a controller of information flow.

\section{B.1.1 Guidelines for multimodal interfaces design}

During the development of multimodal systems, the user characteristics, input facilities and context features must be taken into account to get effective multimodal interfaces. Several authors have given guidelines for successful MMI design and the following is a summary focusing on for mobile devices (from Chittaro [6], Dumas [19] Oviatt [46] and Reeves [50]):

- MMI design should cover most of the target system users by giving different input/output modalities. Such modalities let the system adapt to different situations, user preferences, capabilities and needs.

- Security and privacy are essential aspects in multimodal interaction. Multimodal systems should offer input modes that do not expose private user information in insecure locations.

- The correct combination of input modes could reduce the cognitive work load of the user and increase the usability of the system.

- Not all multimodal inputs include simultaneous signals, so the input processing could be sequential or in parallel.

- Different input modes are not able to transmit comparable content.

- Try to reduce the amount of information shown in small screens.

- Aesthetics is a relevant aspect in mobile device interfaces, users tend to choose devices with interfaces visually pleasing.

- Feedback is important in multimodal interaction, users must know the current status of the system as well as the available input modalities.

- Provide exit options for input modalities to let the user change between these. 


\section{B.2 Speech Recognition}

This section provides an overview of the speech recognition history and a theoretical background of this area, including approaches for signal processing and speech recognition.

\section{B.2.1 History of speech recognition}

Transcribing utterances to text is not a recent challenge. Current automatic speech recognition (ASR) systems with large databases and low word error rates have their roots in the inspiration to simulate human behavior on machines.

Since the late 1800s, there were approaches to reproduce human speech in works such as von Kempelen's "Speaking Machine" [18], but it was not until 120 years later that Bell Laboratories reported the relationship between the speech spectrum and its sound characteristics [33]. This important relationship resulted in the implementation of different speech recognition algorithms based on speech power spectrum measurements. Some decades after that (1950-1970), science fiction works such as Isaac Asimov's novels, the television series Star Trek (1966), and the movie 2001: A Space Odyssey (1968) increased the interest in human-machine interaction, specifically, the use of the voice as a tool to communicate with computers and control them. Relevant advances were reached in this period. In 1952, an ASR system for isolated digit recognition was built using formant frequencies, which are spectral peaks manifested in the speech power spectrum [33]. In 1962, the article "The phonetic typewriter" [53] was published containing an analysis of different utterance portions using a speech segmenter, an early approach for continuous speech recognition systems [33].

Tom Martin's work in dynamic programming for speech pattern aligning in non-uniform time scales [41] had an impact on the Sakoe and Chiba research on dynamic time warping (DTW) [33], an important concept used in conjunction with different search techniques such as the Viterbi algorithm, where extracted features are compared with previous models in order to find the corresponding word. With the aim of developing speaker-independent systems, AT\&T Bell Laboratories developed algorithms to represent sound patterns for words with a vast number of samples. This work culminated in the development of statistical modeling techniques [33]. Such techniques were the basis for hidden Markov models, currently the most common approach in the field.

Until the 1980's most speech recognition methodologies used template-based pattern recognition. In this decade the statistical approach emerged with the use of hidden Markov models (HMMs), a framework developed at AT\&T Bell Laboratories and based on research at the Institute for Defense Analysis (IDA) in the late 1960's and James Baker in 1975 [3]. HMMs became the most popular method because they are robust for continuous recognition and variation in time, and allow speaker-independence capabilities.

Also in the 1980s, an alternative technology was proposed. From the field of artificial intelligence came the use of artificial neural networks (ANNs). From the point of view of pattern recognition, a multilayer perceptron, a particular form of parallel distributed processing, could perform the mapping between utterances and words with an acceptable accuracy level, following a training process using the back propagation method of ANNs. This approach gave good results for isolated word recognition, but not for continuous words with large data sets because of the temporal variation on speech recognition [33].

\section{B.2.2 State of the art}

The last two decades have seen great advances in the improvements in HMMs and implementations of robust automatic speech recognition systems in academia, the military, and industry. The following is a brief description of some of the more relevant speech recognition systems, focusing on open source systems. 
Sphinx: In 1988, the first version of Sphinx was released [36]. Sphinx is an open source HMM-based speaker-independent speech recognition system. Since this early version, Sphinx has evolved. Sphinx-II (1993) reduced the word error rate from $20 \%$ to $5 \%$ on a 5000 word vocabulary by using a data-driven unified optimization approach [28]. In 1997, the new Sphinx-3 could be configured at run-time along the spectrum of semi- to fully-continuous execution [48]. Sphinx-3 was developed in $\mathrm{C}++$ and is still in active development. Pocketsphinx, a Sphinx-II version developed for hand-held devices, was released in 2006 [29]. This version was designed for continuous speech recognition on devices with low hardware capabilities. Despite its features, Pocketsphinx has only been tested on desktop computers and more recently on Linux-based mobile devices [62] and on the iPhone. The most recent version of the Sphinx series is Sphinx-4, developed in Java [65], which presents a modular framework for HMM-based speech recognition systems. Sphinx has been the base of many projects, including Lumenvox, Microsoft Whisper, Jvoicexml, Gnome-Voice-Control and Zanzibar [8].

HTK: The Hidden Markov Model Toolkit (HTK) was developed to support hidden Markov model processing, primarily for speech recognition tasks. The Speech Vision and Robotics Group of Cambridge University released the first version in 1989 which supported diagonal and full covariance Gaussian mixture distributions for observation probabilities. In 1995, HTK became part of the Entropic Cambridge Research Laboratory (ECRL). In this year, a redesigned platform was released as version 2.2, which supported discrete density HMMs, cross-word triphones, lattice and N-best recognition output, back-off bigram language models and decision-tree state clustering. Microsoft purchased the ECRL in 1999, and all HTK rights went to Microsoft. In September 2000, the source code was released with the aim to improve the system, and to let researchers and developers around the world participate in project development. The current version (3.4) has an improved set of features for speech recognition such as perceptual linear prediction (PLP), N-gram language modeling, speaker adaptive training, discriminative training and large vocabulary decoding [5].

Julius: An open source continuous speech recognition system for large vocabularies, Julius was initially developed at Kyoto University in 1991, and from 2005 was copyrighted by the Nagoya Institute of Technology. This system performs recognition with up to 60K words, and it has a recognition rate of $90 \%$ in real time. The system uses $\mathrm{N}$-gram language models and HMM acoustic models, and it is widely compatible with HTK file representation formats (HMM definition and model files, feature parameters). Desktop Linux is the target platform, but there are versions for Windows and Solaris [42]. An embedded Julius-based version was developed to run on a T-engine board with a $240 \mathrm{MHz}$ RISC processor, a developmental hardware platform [35]. A desktop speech recognition system for Brazilian Portuguese based on Julius was developed at the Brazilian Federal University of Pará [57].

Commercial applications: A broad range of commercial speech recognition systems are available. Most of these are HMM-based and compatible with desktop computers. Some of the systems are: Byblos, developed by Raytheon BBN technologies [7], Decipher, developed at SRI [9], Dragon NaturallySpeaking from Nuance [45], and ViaVoice, an IBM speech recognition system [30].

A common measure for evaluating speech recognition accuracy is word error rate (WER) shown in Equation B.1.

$$
W E R=100 \% * \frac{\text { Subs }+ \text { Dels }+ \text { Ins }}{\# \text { of words in the correct sentence }}
$$

where Subs is a count of substitutions for a correct word, Dels are omitted words and Ins are inserted words [27].

Table B.1 shows typical automatic speech recognition word error rates (WERs) for English tasks as of 2007 for continuous recognition on different speech tasks [54]. Connected digits is a domain of digit strings recorded in a clean environment. The DARPA resource 
management corpus includes military commands, queries, and responses in a clean environment. The recognition task allows no more than 1000 words. The Wall Street Journal corpus includes speech read from newspaper stories in a clean environment, with a 5000 word vocabulary for the testing task. The SWITCHBOARD corpus is spontaneous conversations in a telephone environment, which makes it a more difficult task. The best WERs for typical tasks in this domain are still around $20 \%[20,26]$ depending on the particular test set and modeling approaches. For small vocabularies (e.g., connected digits) and clean environments, accuracy rates close to $99 \%$ are expected. WER increases for larger vocabularies, unplanned or spontaneous speech, multiple speakers and noisy environments.

Table B.1: Current recognition word error rates on desktop ASR systems [20, 26, 54]

\begin{tabular}{|l|l|c|}
\hline Task & Vocabulary Size & WER (\%) \\
\hline Connected digits & 11 & 0.6 \\
\hline Resource Management & 1,000 & 2.7 \\
\hline Wall Street Journal & 5,000 & 7.2 \\
\hline SWITCHBOARD & $30 \mathrm{~K}-40 \mathrm{~K}$ & $\approx 20$ \\
\hline
\end{tabular}

\section{B.2.3 Concepts}

Automatic speech recognition is a process where utterances are translated to character strings with an equivalent meaning. That character string might be a set of digits, a word or a sentence, and can be used as an execution command, to get the text of the speech for subsequent processing, or in automatic dialog or speech understanding systems.

A typical speech recognition system is composed of two phases, a front-end and a backend (Figure B.2). The front-end module processes the voice signal to get observation vectors that are used in training and recognition [4]. The back-end module is comprised of a word search, combining information from acoustic and language models extracted from a training phase.
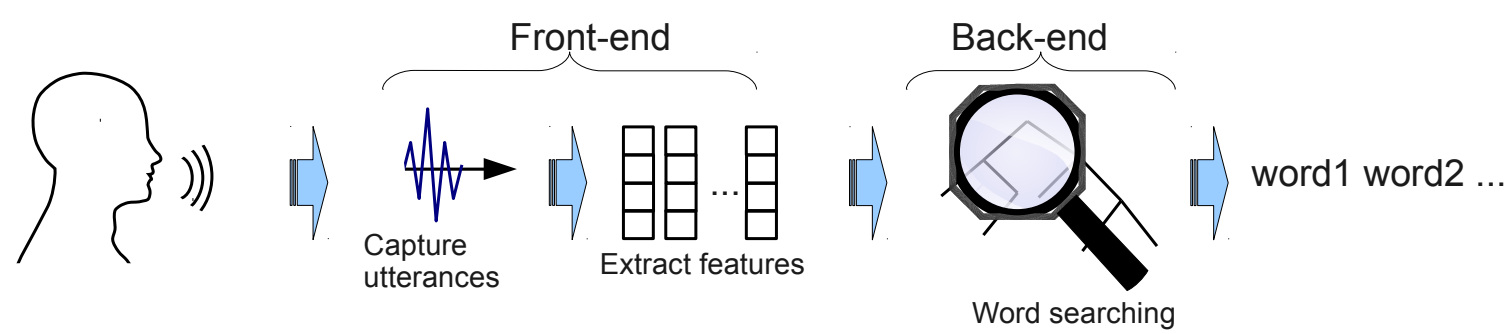

Figure B.2: Speech recognition process

\section{Front End: Signal processing}

In the front-end, voice utterances are digitized to waveforms and subsequently transformed to a parametric representation for further processing. Different approaches are used to get the parametric representation. Filter bank analysis [49], linear predictive coding (LPC) [49], and Mel frequency cepstral coefficients (MFCCs) [27], have been efficient techniques in speech signal feature extraction. Characterization of the signal is based on the source-filter model, a speech production model where the speech $x[n]$ is the result of the interaction of the air from the lungs (source or excitation $e[n]$ ) with the vocal tract (filter $h[n]$ ) in time [27]. Figure B.3 shows the source-filter model. The objective in this stage is to separate the source and the filter so that filter information can be used in recognition. Different techniques to 


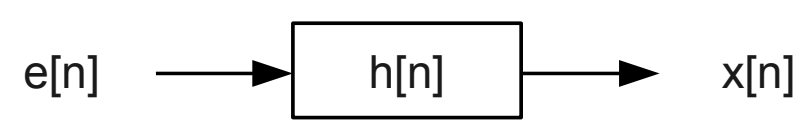

Figure B.3: Speech recognition process

estimate the filter are based mainly on speech production models, such as the filter-bank and LPC, and speech perception models, like MFCC. When the filter is estimated, an inverse filter is used on the speech to get the source [27].

This subsection will briefly describe three common approaches for representing the speech signal for subsequent processing: filter-bank, LPC, and MFCC. These techniques use as their starting points the spectral representation, which contains relevant frequency domain information. In speech recognition, the Fast Fourier Transform algorithm (FFT) is often used to get the spectral representation along with other transformations such as the discrete cosine transformation.

Filter bank approaches divide the frequency of the signal into $\mathrm{N}$ bands to measure the intensity or energy in each one [49]. The energy of the segment is obtained by calculating the area under the curve or summing the squares of the values. Three types of filter banks are used: uniform, logarithmic, and non-uniform. A uniform filter bank divides frequency into uniform intervals, losing useful signal characteristics because lower frequencies carry more information. Logarithmic filter banks divide the signal logarithmically, simulating human auditory perception. If the first range has a width $W$, then subsequent ranges will be $a^{n} W$, where $a$ is a logarithmic growth factor. In non-uniform filter banks, ranges are composed of two parts. In frequencies from $100 \mathrm{~Hz}$ to $1 \mathrm{KHz}$ ranges are spaced equally; from $1 \mathrm{KHz}$, ranges will be divided with the logarithmic scale. This partition is known as the Mel scale [27], and it is widely used in speech recognition because it emphasizes lower frequencies.

Linear predictive coding coefficients (LPC) estimates the value $x[n]$ of a signal, by combining the past $p$ speech samples [27]. So if we have

$$
x[n]=\sum_{k=1}^{p} a_{k} x[n-k]+E[n]
$$

where the coefficients $a_{k}$ are constants over the speech frame, and $E[n]$ is the prediction error. Then, the estimated signal is given by Equation B.3

$$
\tilde{x}[n]=\sum_{k=1}^{p} a_{k} x[n-k]
$$

and the error is given by Equation B.4

$$
E[n]=x[n]-\tilde{x}[n]=x[n]-\sum_{k=1}^{p} a_{k} x[n-k]
$$

The aim here is to estimate the $p$ coefficients that minimize the prediction error $E[n]$. A solution to estimate such coefficients is the Yule-Walker equation [27]:

$$
\sum_{j=1}^{p} a_{j} \phi_{m}[i, j]=\phi_{m}[i, 0] \quad i=1,2, \ldots, p
$$

where $\phi_{m}[i, j]$ is an LPC coefficient for the sample $m$.

Mel frequency cepstral coefficients (MFCCs) are based on a signal representation 
technique that provides a better characterization than filter banks alone and is currently the state-of-the-art in automatic speech recognition [25]. The MFCC process is based on cepstral analysis, where multiplication of the source and filter components on the frequency domain can be represented as the time convolution of the speech excitation signal and the filter. The source and filter can then be separated readily using basic filtering methods [25]. MFCCs are based on speech perception models, that is, a nonlinear frequency scale used to simulate the human auditory system. The MFCC process starts in filter-bank analysis, where a discrete cosine transformation is applied to the Mel filters. The resulting MFCC will maintain the characteristics of the non-uniform filter-bank and a smaller element vector will be needed to represent such information. Additionally, higher coefficients are removed, allowing faster computation in subsequent processing [25].

Once the signal representation has been selected, a distance metric must be defined to measure the similarity of the captured signal with previous data. Given parameter vectors of $N$ elements, a real Cartesian space $R^{N}$ will contain all real elements of the parameter vectors. Distance measures will be computed over this space. Three distance types are commonly used: City block, Euclidean, and Itakura metrics [51].

\section{Back End: Speech recognition approaches}

Once signal processing is performed, the next stage is to recognize the utterance, based on a previous training phase. Training is where characteristics of utterance samples are extracted to create models and later map new sounds to these models. Training depends on the recognition approach and can require generating templates (template matching) or acoustic models (e.g., hidden Markov models) coupled with language models (finite state grammars or $N$-gram models). In recognition, the system performs a mapping process to retrieve the closest word to the incoming utterance. Figure B.4 illustrates the recognition in the back-end.

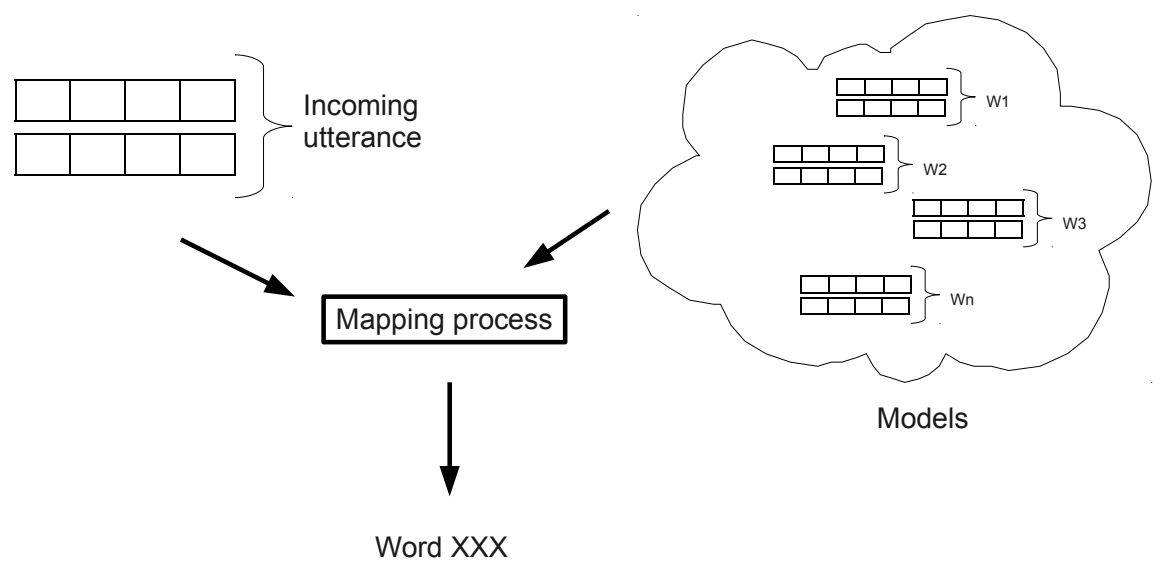

Figure B.4: ASR Back-end: recognition phase

How a speech recognition system identifies utterances depends, initially, on the recognition type, whether isolated or continuous words are recognized, and secondly, how the system performs the recognition, that is, using template matching or statistical modeling techniques. Isolated word recognition refers to the identification of one word at time. Systems listening for commands or simple words are good candidates for isolated word recognition because each utterance will be related to a specific word or short phrase. Continuous recognition refers to the determination of word tokens in continuous audio streams. In this case, the system will process longer utterances, identifying the end of a word and the beginning of the next. Examples of this type of recognition are dictation systems and live translation applications. 
In continuous recognition, two approaches have been used to recognize speech. The wholeword method, where the system has models of whole words to match the speech, only recognizes words that have been previously modeled. Phone recognition, where utterances are divided into sub-word segments, uses a dictionary, mapping these segments, called phones, to words. The system is able to identify previously seen words as well as words not seen but composed of phones which have been seen in training data.

Template matching is a pattern matching technique used mainly during the 1970s and 1980s. Foundations of this technique came from the fact that when a person says a word several times, each signal representation will follow a pattern which can be used to identify an utterance in a speech recognition system. Thus, if the system has the acoustic patterns (templates) of any supported word, the template matching method will find the closest template to the signal representation of the incoming utterance. Basically, the search consists of selecting the template with the lowest distance from the utterance. Euclidean distance is a common metric used in this technique. While this technique can work in simple applications, there are some limiting factors. First, while several recordings of a word from a single person will give us a pattern, it will not be valid for any other person. Thus, template matching is user-dependent, and each user must train the system by giving examples of the supported words. Second, this technique assumes that a word is completely defined, that is, the start and end of the word are readily determined. For isolated recognition this is not a problem, an utterance will match with a word. However, continuous word recognition must have an extra processing phase to divide the signal into individual words.

Time and pitch variations in utterance representations can be found in recordings from the same person. Word segments may vary in duration. For example, the word "computer" can be said as "compuuter" or "commputrr", both have a different syllable lengths but the same meaning. To deal with this time variation, a mathematical technique known as dynamic programming or dynamic time warping (DTW) is used. DTW minimizes the cumulative distance between two strings, but avoids the calculation of all possible combinations [25]. Figure B.5 shows the optimal path between "computer" and "commputrr". Point $i, j$ represents the lowest distance in this segment of the words. The minimal cumulative distance is given by Equation B.6.

$$
D(i, j)=\min [D(i-1, j), D(i, j-1), D(i-1, j-1)]+d(i, j)
$$

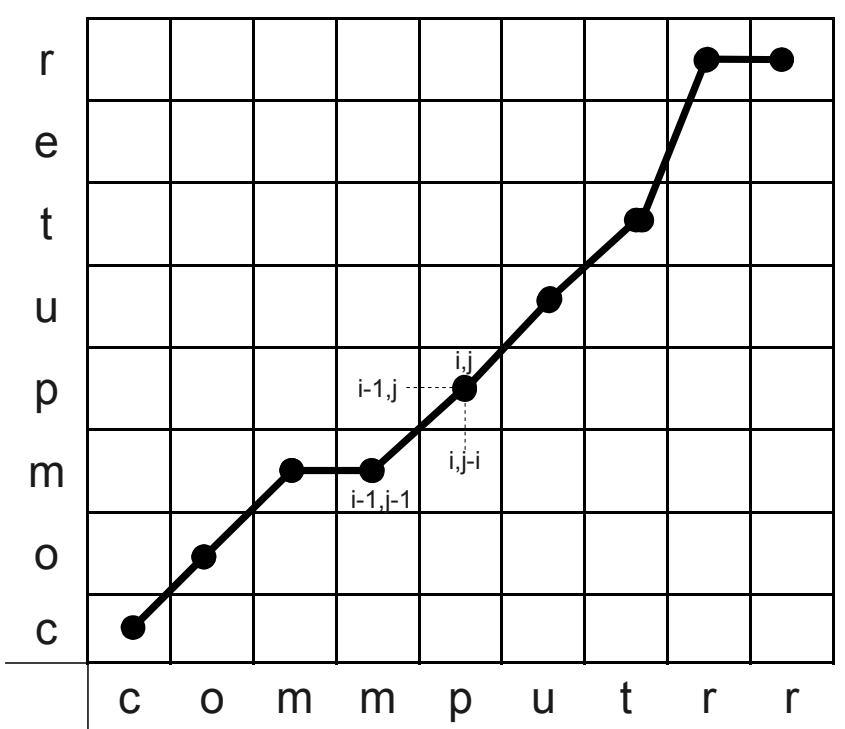

Figure B.5: Dynamic Time Warping 
For continuous recognition, the main problem is the end-detection of single words. In this case, template matching has been carried out by concatenating templates and performing the DTW algorithm on the entire sentence. To separate templates and identify words, a silence template is included between normal templates. Wildcard templates are used in sentences where the end of the word is not identifiable.

Statistical approach: A second approach for the back-end phase is statistical modeling using a combination of acoustic and language models. While template matching is a useful methodology for isolated-word recognition, word boundary identification is difficult for connected-words and continuous speech. Speech variations such as pitch, length, internal noise (vocal tract variation), environmental noise and co-articulation effects at word boundaries make sentences harder to recognize. In the 1970s, hidden Markov models (HMMs) were proposed to confront these acoustic issues [3]. With HMMs, it is assumed that models can generate feature vectors of any supported word in the system [25]. The recognition process evaluates the a posteriori probability that a model could generate the same feature vectors as incoming utterances, but without actually generating such vectors [25]. The likelihoods of different model sequences can then be compared to choose the most likely hypothesis that could have generated the test sentence

Taking as an example an isolated word, which eliminates boundary problems, and given a set of feature vectors or observations $O$, defined as the set of observations along time $t$, $O=o_{1}, o_{2} \ldots, o_{t}$; the recognition problem is to find the highest probability for a particular word given a set of observations, or $P\left(w_{i} \mid O\right)$, and is defined by Equation B.7

$$
\underset{i}{\operatorname{argmax}}\left\{P\left(w_{i} \mid O\right)\right\}
$$

where $w_{i}$ is a word in the set of all possible in the system [68]. Using Bayes' rule, the probability distribution in Equation B.7 becomes:

$$
P\left(w_{i} \mid O\right)=\frac{P\left(O \mid w_{i}\right) P\left(w_{i}\right)}{P(O)}
$$

Given a set of prior probabilities $P\left(w_{i}\right)$ represented by the language model, Equation B.8 is reduced to the computation of $P\left(O \mid w_{i}\right)$, that is, $P\left(o_{1}, o_{2} \ldots \mid w_{i}\right)$, an intractable problem because of the dimensionality of the vectors [68]. Here, the HMM is introduced to replace the $P\left(O \mid w_{i}\right)$ with a simpler solution. From the HMM diagram (Figure B.6), the $o_{t}$ vector is generated from the probability density $b_{j}\left(o_{t}\right)$, and the transition from a state $i$ to a state $j$ has the probability $a_{i j}$. The joint probability that $\mathrm{O}$ is generated by the model $\mathrm{M}$ is:

$$
P(O, X \mid M)=a_{12} b_{1}\left(o_{1}\right) a_{22} b_{2}\left(o_{2}\right) a_{23} b_{3}\left(o_{3}\right) \ldots
$$

where $X$ is the unknown sequence through the model states. $X$ is calculated by summing over all state sequences $X=x(1), x(2), \ldots, x(T)$. Thus,

$$
P(O \mid M)=\sum_{x} a_{x(0) x(1)} \prod_{t=1}^{T} b_{x(t)}\left(o_{t}\right) a_{x(t) x(t+1)}
$$

with $x(0)$ being the initial state and $x(T+1)$ the final state. The solution for Equation B.10 is based on recursive procedures [68]. Finally, Equation B.8 is solved by assuming that $P\left(O \mid w_{i}\right)=P\left(O \mid M_{i}\right)$. Parameters $a_{i j}$ and $b_{j}\left(o_{t}\right)$ are estimated by performing training over all possible words in the training set. This process generates a model that represents the acoustics associated with real words. Baum-Welch re-estimation is used to calculate the HMM parameters [68]. The Viterbi algorithm performs the search for the $\operatorname{argmax}_{i} P(O \mid M i)$ to get the closest model sequence to the unknown sentence [68]. 


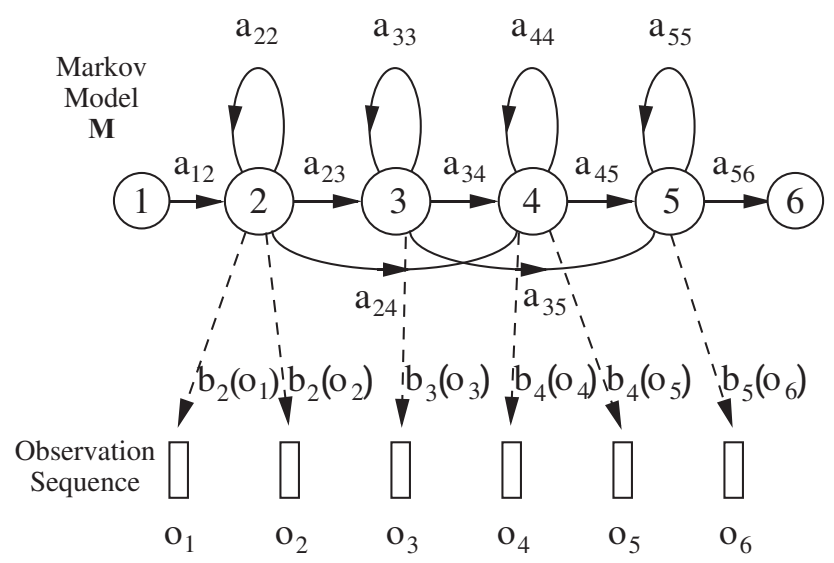

Figure B.6: Markov generation model [68]

The global model $M$ is characterized by the number of states in each component model, the size of the observation symbols, transition probability matrices $A=a_{i j}$, output probability matrices $B=b_{i}(k)$, that represent the probability of emitting symbol $o_{k}$ when a state $i$ is reached, and an initial state distribution $\pi, M=\{A, B, \pi\}$. In practice, $P\left(O \mid M_{i}\right)$ is an acoustic model, where $B$ is a discrete output probability matrix for finite sets of (isolated) observations, or an output probability function for continuous recognition. Multivariate Gaussian mixture density functions are commonly used for the output probability function, because of their capability to approximate any probability density functions [27]. To improve the accuracy in the system, a large number of mixtures can be chosen, but this increases the computational complexity. On the other hand, discrete models have low computational complexity, but low performance levels. Semi-continuous models provide a good balance between system accuracy and computation level [27].

Semi-continuous and continuous speech recognition are dealt with by concatenating HMMs, where boundaries are represented by initial and final states. Similarly to template matching, boundary detection is a common issue. Typical HMM training uses a pre-training phase, called bootstrapping [68], to detect boundaries using Baum-Welch re-estimation in parallel. Connecting whole-word HMMs can be used to create sentences and connecting phone models can be use to create words and/or sentences.

When a search is being executed, the system must find the closest model sequence to the input utterance. To reduce the search space and improve the performance, a priori knowledge of the language structure can be used. The probability $P(W)$ represents the likelihood that the word string $W$ corresponds to the utterance, and is defined by the language model. Here, a language model is a probabilistic model of the all possible sequences in the system. Stochastic language models, such as the $n$-gram, are commonly used in speech recognition where sequences of $n$ words are used rather than all possible sequences. An $n$-gram model for a sequence of words fo length $I$ can be described as

$$
\begin{aligned}
& P(W)=P\left(w_{1}, w_{2}, \ldots, w_{I}\right) \\
& P(W)=P\left(w_{1}\right) P\left(w_{2} \mid w_{1}\right) P\left(w_{3} \mid w_{2}, w_{1}\right), \ldots, P\left(w_{I} \mid w_{I-1}, \ldots, w_{2}, w_{1}\right) \\
& P(W)=\prod_{i=1}^{I} P\left(w_{i} \mid w_{i-1}, \ldots, w_{2}, w_{1}\right)
\end{aligned}
$$


Limiting $n$ to a value like 3 reduces the size of the model.

$$
P(W)=\prod_{i=1}^{I} P\left(w_{i} \mid w_{i-1}, w_{i-2}\right)
$$

\section{B.3 Brazilian Portuguese corpora}

To train and test speech recognition systems, a data set of utterances and their transcriptions for a specific language must be available. There are only a few sources for Brazilian Portuguese speech corpora, although there has recently been work to augment this. Spoltech is a Brazilian Portuguese corpora with 8119 utterances from the Oregon Graduate Institute (OGI) and the Rio Grande do Sul Federal University (UFRGS) [15]. OGI-22 is another available corpus that consists of telephone speech from 22 languages [14]. This is a smaller corpus and only a few utterances have orthographic transcriptions. Previous work with these two corpora has been carried out at the Federal University of Pará. This work showed word error rates of $18.6 \%$ and $19.9 \%$ for Spoltech and OGI-22 respectively [44]. Globalphone is another multilingual corpus collected at Karlsruhe University [61]. For the Brazilian Portuguese version, the corpus was created by having 102 speakers read from the Folha de São Paulo newspaper. Globalphone is available under a commercial license which was too expensive for this project and would not allow open source licensing for the resulting code. VoxForge is a recent initiative to make available free resources for speech recognition [63]. Here, different corpora and acoustic models are available under GPL licensing. A Portuguese data set with a total of 51 minutes is available. All available corpora consist of vocabularies of commonly used words. Many utterances were elicited with requests for addresses, phone numbers, and personal information. While the breadth of speakers is useful, the specific vocabularies do not meet the needs of Borboleta, which uses speech in the healthcare domain.

\section{B.4 The Borboleta telehealth system}

The Borboleta telehealth system is a mobile application to collect medical information during residential visits in the homecare program provided by the University of São Paulo Primary Healthcare Center [16]. The Borboleta system is composed of two modules, a mobile component to support the visits and SAGUISaúde, the central application that manages and provides the medical information in the health center. Figure B.7 shows the overall architecture of the Borboleta system. To conduct the medical visits, the mobile device is synchronized with the server by downloading the medical information for the scheduled visits. The visit is conducted and the data is saved in the mobile devices. The collected information is then uploaded to the server and can be accessed using a Web browser.

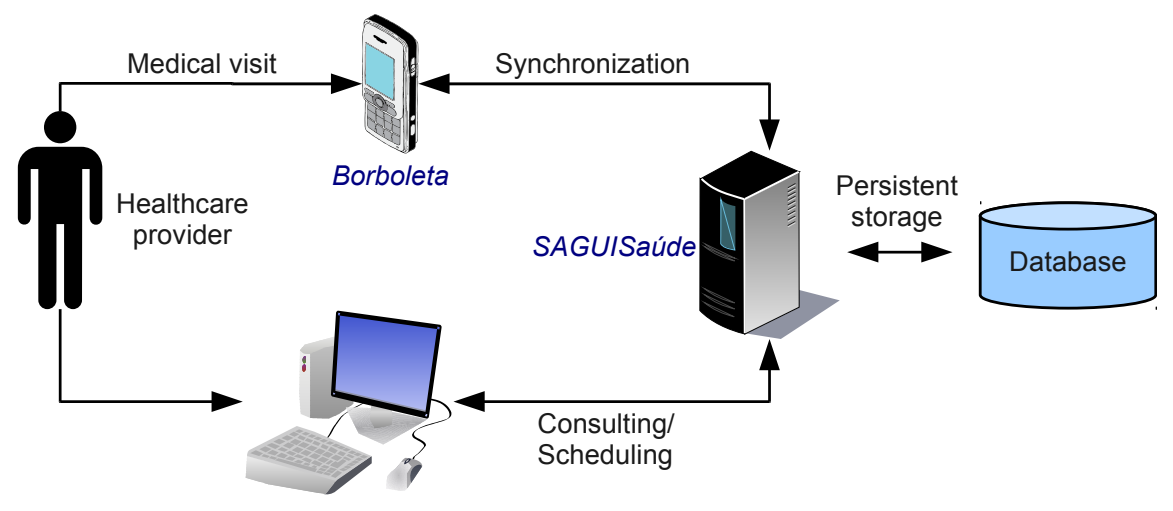

Figure B.7: Borboleta - Overall architecture 
Because of the complex and varied medical information that must be collected, the Borboleta mobile module offers different input screens as well as defined procedures to complete a task. The main features of the system are collection of patient and caregiver data, visit scheduling, and collection of information about the residential visit, patient status, problems and needs. The system provides a synchronization schema using XML over an SSL connection through a wireless network. Figure B.8 shows some screenshots of the complex graphical interface of the Borboleta software.

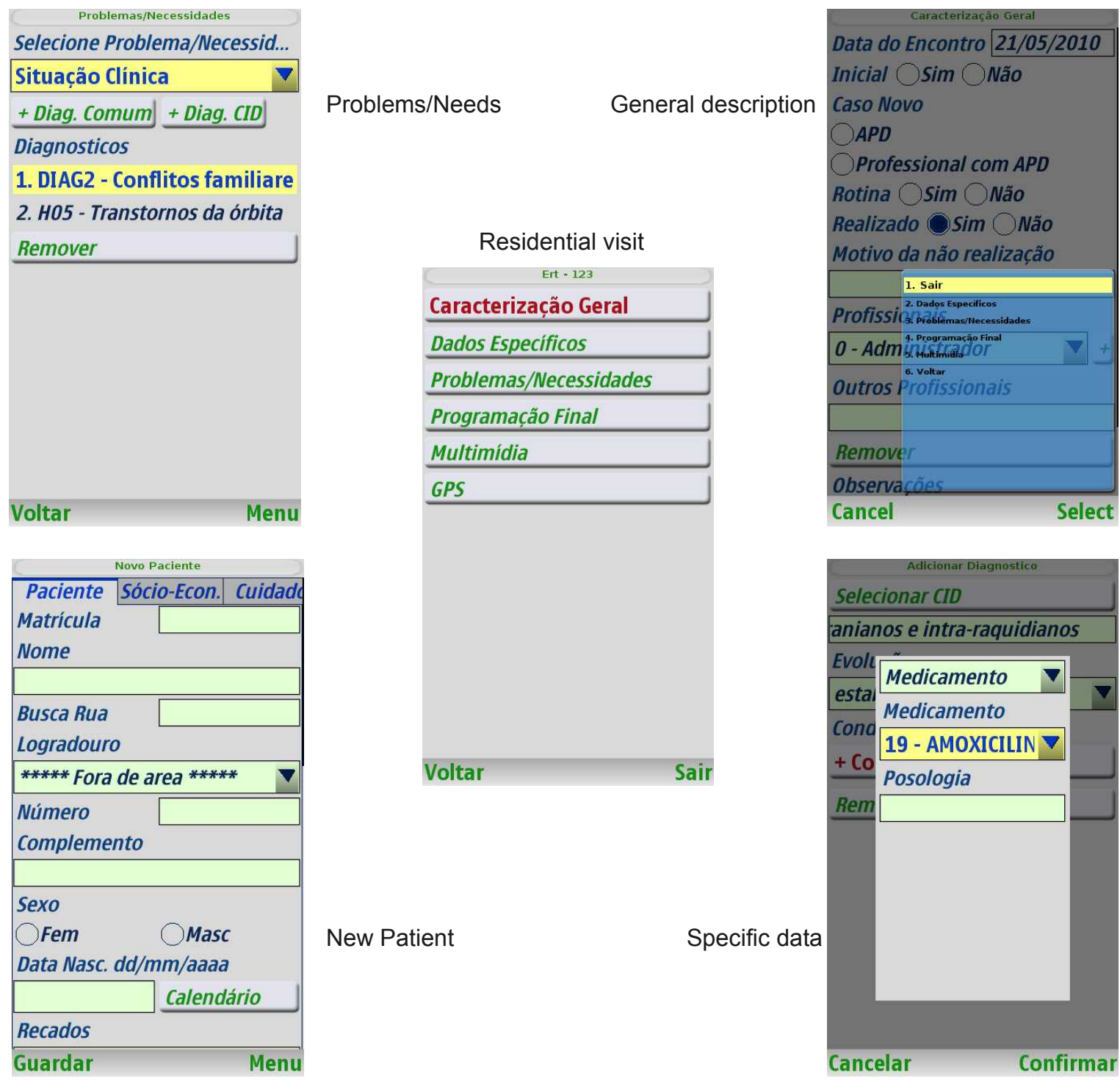

Figure B.8: Borboleta - Interface screens

The system has been developed in Java for the Java ME specification in order to guarantee the highest compatibility with mobile devices. Currently the system runs on Windows Mobile, Palm OS, Android and Symbian, but new multimedia features are supported only by the Nokia devices (Symbian S60).

\section{B.5 Mobile devices}

This section describes mobile devices and their platforms, followed by speech recognition approaches for mobile devices and current available software. Finally, the Borboleta system architecture is briefly described. 


\section{B.5.1 Mobile devices and platforms}

Mobile devices are portable electronic devices that can work in many environments. Typically, mobile devices have lower size and weight than normal computers. In today's market, there are a wide range of mobile devices, such as notebook computers, personal digital assistants (PDAs), mobile phones, calculators, game consoles, e-book readers, and portable media players. In this case, we will examine Borboleta compatible devices, that is, PDAs and mobile phones running Java mobile edition (Java ME). For our target devices, common characteristics include a processor with speeds between $300 \mathrm{MHz}$ and $1 \mathrm{GHz}$, RAM from $32 \mathrm{MB}$ up to 512MB, storage (internal and/or external) from 512MB up to 16GB. For input/output methods, the devices are typically equipped with 4" touch screens, mini-qwerty keyboards, accelerometers, GPS, and audio and video capture/player libraries. The devices normally come with GSM and UTMS networks (mobile phones), wireless network connectivity (Wi-Fi) and bluetooth capabilities.

In the same manner as desktop operating systems, mobile platforms abstract hardware access and manage system resources, but mobile platforms take into account additional requirements, such as battery life optimization in resource usage. Similar to the wide range of mobile devices available, many different platforms are available. Typically, mobile platforms are compatible with a specific set of devices. It is not possible to install another operating system (OS) on a device if the new one is not compatible with the device. This issue causes device dependence on specific OSs, and execution of applications developed for a particular platform. Java ME was developed to address the portability problem. This platform is a virtual execution environment or virtual machine that guarantees a high compatibility level among different mobile platforms by adding virtualization middleware between the OS and the application. The interaction between application and virtual machine will thus be the same across all platforms but, between the virtual machine and the OS, there will be specific implementations. Symbian, RIM Blackberry, Windows Mobile, iPhone OS, Android and Palm OS are some of the more relevant operating systems for mobile devices. Figure B.9 shows the market distribution for mobile operating systems for the first quarter of 2011 .

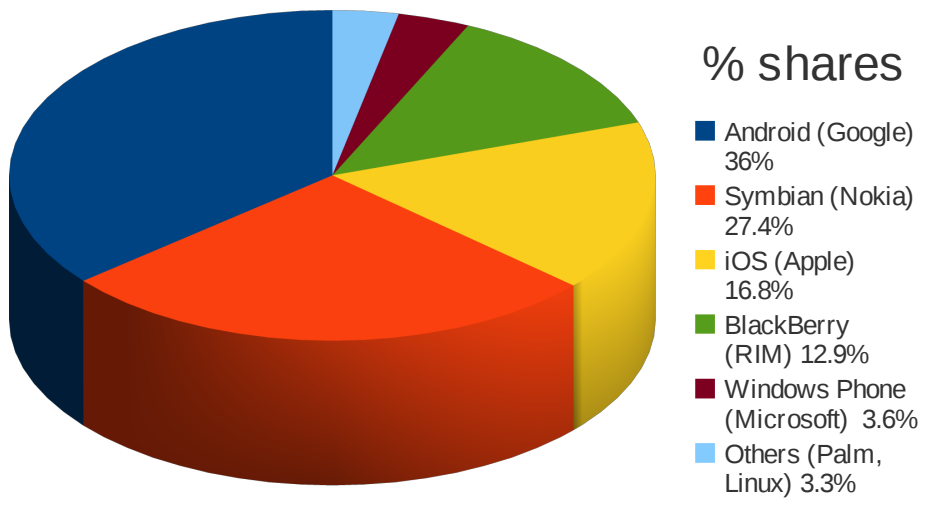

Figure B.9: Mobile OS world market Q1 2011 [23]

Symbian, which has one of the largest global market shares, is an OS for mobile devices and is primarily used by Nokia and Sony Ericsson devices. Symbian was developed in C++ and recently was released as open source. Symbian architecture, as shown in Figure B.10, was designed as a multilayer system, where each layer offers specific functionalities and is able to communicate with the tier below. Native applications run on the S60 applications layer using the language Symbian $\mathrm{C}++$, an implementation of ANSI C++ optimized for mobile devices. Since most of the devices used in the Borboleta project were Symbian-based, the porting and integration was focused on this platform. Pocketsphinx has been also ported 
to Android OS, which guarantees that the generated models used in the Symbian platform could be used on Android OS. The generated code in Qt/C++ could also be used on Android thanks to the Necessitas project. ${ }^{1}$

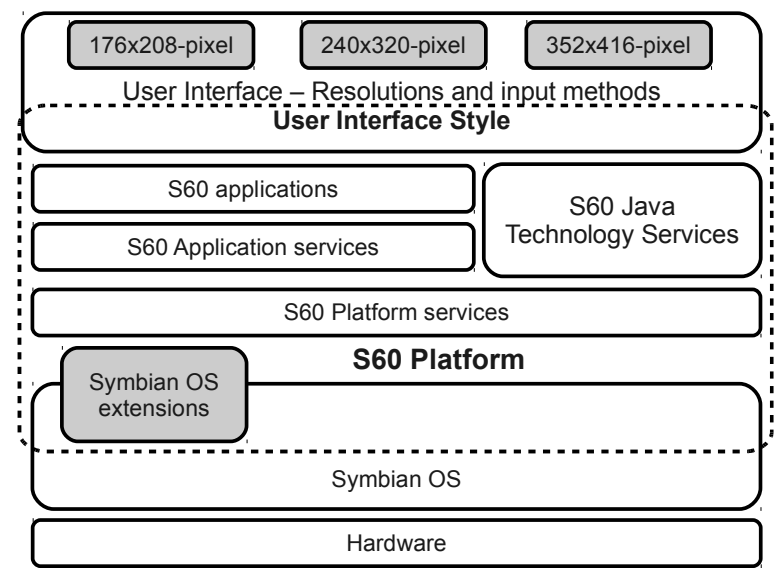

Figure B.10: Symbian architecture [66]

The Borboleta project has been developed for Java ME compatible devices to maintain compatibility with most of the mobile platforms. Java ME provides two configurations for two ranges of devices. The first is connected limited device configuration (CLDC) for devices with low resources, such as early generation mobile phones and PDAs. The second is connected device configuration (CDC) which is used in devices such as new line mobile phones and PDAs with at least $200 \mathrm{MHz}$ processors, multimedia enabled and with a wireless connection. Figure B.11 shows the Java ME architecture for both configurations. Despite the high level of compatibility, not all Java ME implementations provide all the specifications of Java ME. Some implementations offer functionalities for basic operations, but do not support advanced tasks like multimedia processing. For example, multimedia functions like capturing video and audio are enabled in Nokia cellphones (Symbian OS), but unavailable for HTC mobile phones (Windows Mobile) [12].

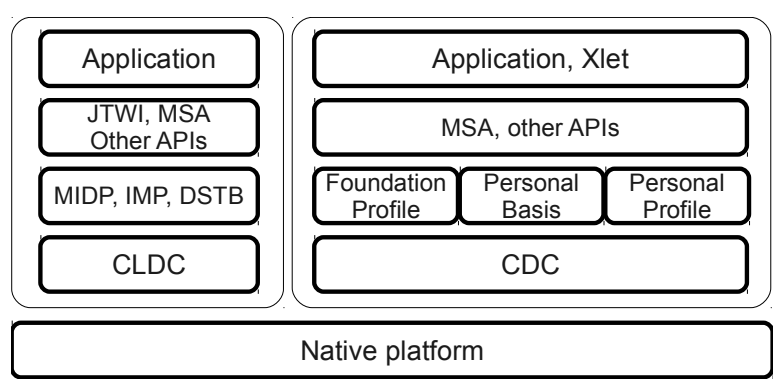

Figure B.11: Java ME architecture [58]

Most automatic speech recognition (ASR) systems have been developed in $\mathrm{C}$ and $\mathrm{C}++$ because of its high performance. Thus, in porting ASR systems to mobile devices, the software performance and language compatibility must be taken into account. Performance wise, $\mathrm{C}++$ has an advantage over Java ME because it runs natively on the device, as opposed to Java ME, which is interpreted. Therefore, $\mathrm{C}++$ directly accesses OS functionalities. For language compatibility, mobile OSs are mainly written in $\mathrm{C}++$, so most of these devices could support speech recognition operations, with some modifications for specific platforms

Developing exclusively for Symbian brings portability problems with other platforms because of its specific syntax and data types. An approach to this issue is the portable operating

\footnotetext{
${ }^{1}$ http://sourceforge.net/p/necessitas
} 
system interface (POSIX), an initiative to unify the interface of the various Unix libraries. This same approach has been carried out for mobile platforms like Symbian, which currently can support applications developed for Linux ports [67]. Open $\mathrm{C} / \mathrm{C}++$ is a set of libraries developed by Nokia to make the porting process to Symbian easier. Table B.2 shows the available libraries compatible with POSIX, although it is important to clarify that not all libraries are $100 \%$ compatible.

Table B.2: POSIX libraries for Symbian

\begin{tabular}{|l|l|}
\hline Implementation & Supported libraries \\
\hline P.I.P.S & libc, libm, libpthread, libdl \\
\hline Open C & P.I.P.S, libz, libcrypt, libcrypto, libssl, libglib \\
\hline Open C/C ++ & Open C, IOStreams, STL, Boost \\
\hline
\end{tabular}

\section{B.5.2 Speech recognition on mobile devices}

Because the speech recognition process consists of two phases, signal processing and recognition, it can be decoupled. Possible approaches for implementing automatic speech recognition (ASR) on mobile devices include embedded terminal systems (ETS), network speech recognition systems (NSR) and distributed speech recognition systems (DSR) [69]. An ETS (Figure B.12.a) has full ASR embedded in the device. This system often has a small vocabulary, but does not require external communication. In the NSR (Figure B.12.b), the front-end and the back-end are on the server and the mobile device simply transmits the speech signal to the server. Results could then be returned to the mobile device. The main advantage of an NSR is that the server has more powerful resources, which allow the use of large vocabulary systems with low word error rates. On DSR systems (Figure B.12.c), the front-end is embedded in the mobile device and the back-end is on a server. The device extracts the observation vectors and sends these to the server where they are recognized. An advantage of DSR over NSR is the low bandwidth used in the communication process because the vectors are smaller than the waveforms.

\section{B.5.3 Available ASR software for mobile devices}

Despite the fact that speech recognition has had great advances in recent years, this technology has not been widely used on mobile devices. Speech recognition requires high processing power and resources that mobile devices did not have until recently. Approaches to achieve speech recognition on mobile devices began with template matching techniques, where the application had to be trained for specific users. Speaker dependence has undesirable effects on the use of this technology, as not all users are willing to train the system. If a new user wants to use a trained system, it will not be compatible with his voice. If a system is trained for many users, many templates must be saved and matching across the entire set uses too many computing resources. Currently, mobile devices are equipped with good processing units and many available resources, increasing the interest in developing more sophisticated applications that could be able to use statistical modeling and reduce the speaker dependence. PocketSphinx and PocketSUMMIT are two speech recognition applications for mobile devices that are part of this recent initiative. A front-end Java ME implementation for a DSR was carried out by Zaykovskiy [70].

Pocketsphinx is a HMM continuous speech recognition system for hand-held devices developed by the Carnegie Mellon University Language Technology Institute [29]. The system is based on Sphinx-II [28], but with the triphone-senone mapping representation from Sphinx-III [48]. To run on mobile devices, the software includes memory optimizations to reduce the use of RAM, including data alignment access for modern CPUs and fixed-point 


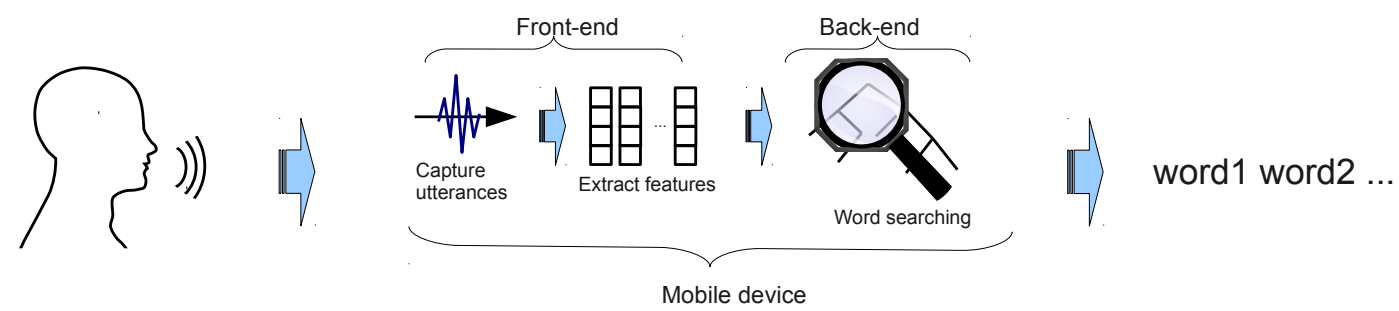

(a) Embedded terminal system

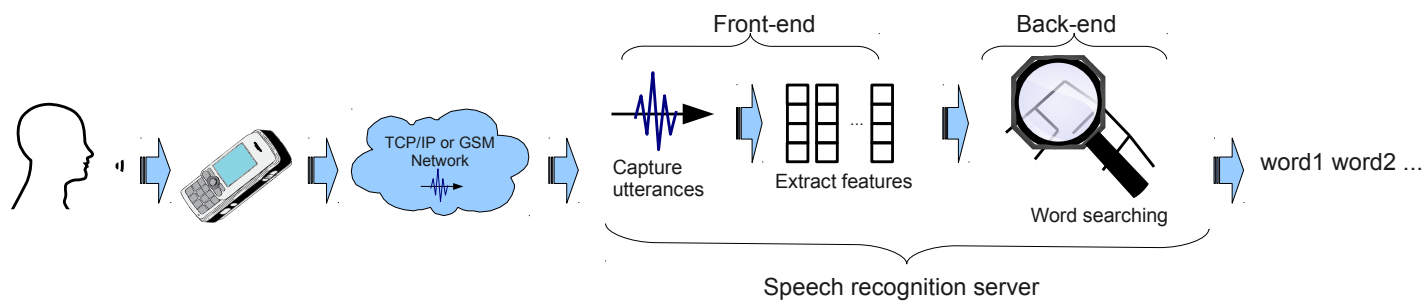

(b) Network speech recognition
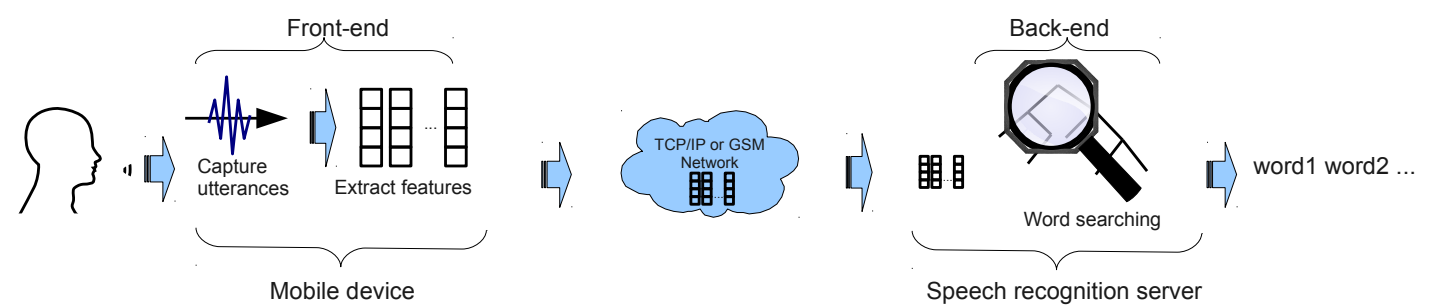

(c) Distributed speech recognition

Figure B.12: Speech recognition approaches for mobile devices

arithmetic because floating-point operations on mobiles are typically emulated. Acoustic models are marked as read only so they are accessed directly using memory-mapped file I/O functions, further reducing RAM usage. Also, there were modifications in the speech recognition algorithms, primarily in the feature extraction procedures, Gaussian calculations, Gaussian mixture model computations and HMM evaluation. The system was tested on the Sharp Zaurus SL-5500 hand-held computer with a 206MHz processor, 64MB of SDRAM, $16 \mathrm{MB}$ of flash memory and a Linux-based operating system. After all modifications, the word error rate of the system on a 994 word vocabulary was $14 \%$ and operated in 0.87 times real time. A successful example of complete recognition processing on a mobile device is the Parakeet project [62]. Parakeet is based on Pocketsphinx and was developed for a Linux-based (Maemo) mobile device.

PocketSUMMIT is a HMM continuous ASR system for mobile devices developed by the MIT Computer Science and Artificial Intelligence Laboratory [24]. The system is based on SUMMIT, a desktop speech recognition system [59]. Optimizations for this system include the use of only landmark features, an 8:1 compression for Gaussian parameter quantization, and finite-state transducer (FST) size reduction by pruning and simplification of the decoder by a state/word graph in the first pass of search. The system was tested on a Windows $\mathrm{CE} /$ mobile device with a $408 \mathrm{MHz}$ AMR-based processor and $64 \mathrm{MB}$ of RAM. The word error rate ranged from $9.5 \%$ to $10.7 \%$ using a 2000 -word weather information domain. 


\section{B.6 Related work}

Recent improvements in hardware and software technologies for mobile devices have allowed researchers to develop more elaborate mobile applications using multimodal interfaces, expanding the application scope to unexplored fields. This section gives a review of research related to multimodal interfaces on mobile devices, with emphasis on the use of speech recognition as input.

\section{B.6.1 VoiceAssist application}

The VoiceAssist application was developed by Samsung Electronics in collaboration with the Rensselaer Polytechnic Institute in 2006 [37]. The system is configured as an embedded recognition system, with U.S. English as the language target, and uses the Palm operating system. The application implements a voice interface for Palm-based phones to access different functionalities like name dial, command-and-control and to start other applications. VoiceAssist offers four operation types: dial commands (calling functions), promoted applications (recording), 3rd party applications (launching applications) and phone applications (checking functions). Figure B.13 shows the available operations. Some graphical interfaces of the application are shown in Figure B.14. The VoiceAssist team carried out a testing

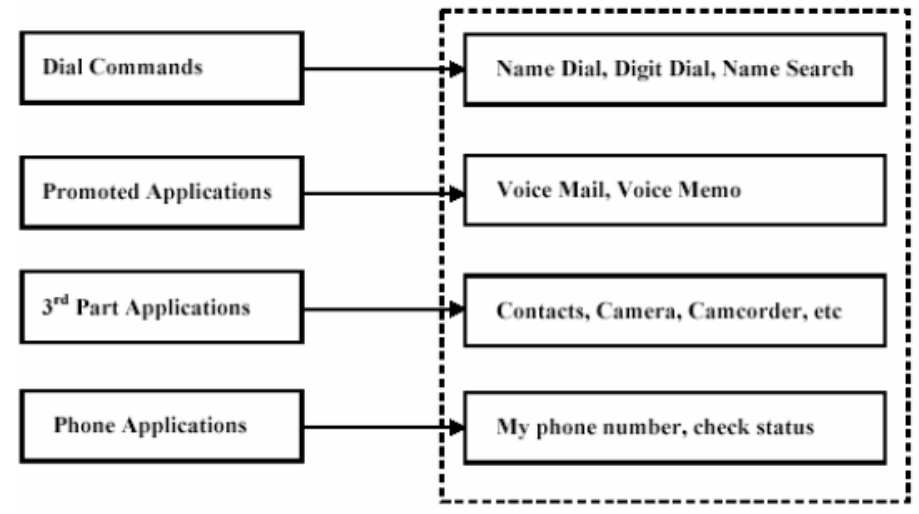

Figure B.13: VoiceAssist operations [37]
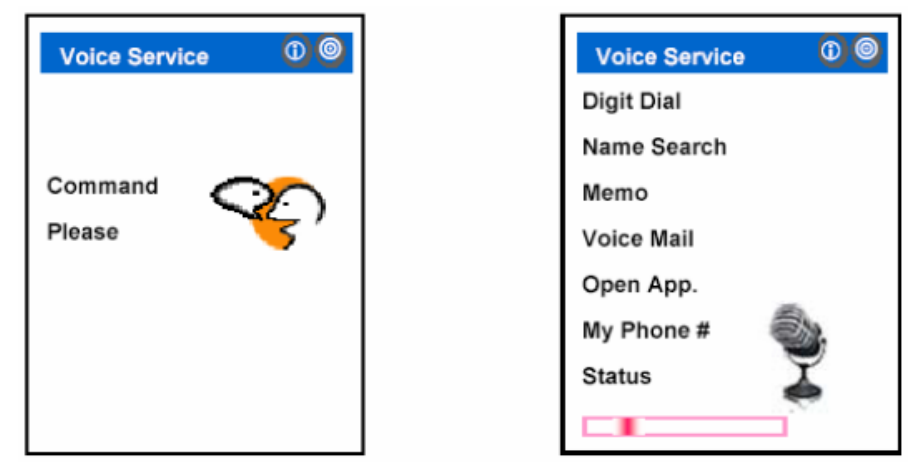

Figure B.14: VoiceAssist interfaces [37]

experiment with approximately 60 participants representing U.S. demographics with ages between 18 and 52 years. They used four testing scenarios, two with a handset microphone with office and car noise, and the last two with a headset with office and car noise. The headset experiment results were $98.6 \%$ and $99.6 \%$ accuracy for commands with office and car noise. Handset microphone accuracy was of $99.7 \%$ for both office and car noise. 


\section{B.6.2 The MONA project}

The MONA project (Mobile multimOdal Next generation Applications) is a presentation server developed at the Telecommunications Research Center of Vienna in 2004. It supports the deployment of device-independent applications with speech input and output capabilities [1]. The general idea is to develop a single user interface for multiple mobile platforms. The MONA server renders a device-specific multimodal interface when a client is connected via 3GSM or a wireless network. The system uses a browser-like approach to create the graphical interfaces and a network speech recognition configuration to guarantee the compatibility of a variety of devices, from low-end WAP phones to Symbian-based smartphones. The MONA project uses a distributed approach for multimodal interfaces where the processing is done on the server side. This requires a continuous network connection.

\section{B.6.3 The ACICARE platform}

ACICARE is a platform for iterative design of multimodal interfaces [55], to allow faster development of MMI and to facilitate the evaluation of the interfaces. ACICARE is based on ICARE, a platform for designing MMI for desktop computers, and on ACIDU, a tool for mobile phones to gather information about functions used and to evaluate multimodal interfaces. The ACICARE was developed to run on Windows CE based phones and was tested on the SPV C500 phone. ICARE, or Interaction-CARE (complementarity, assignment, redundancy, equivalence [55]), proposes a set of steps and concepts to produce effective multimodal interfaces. In the mobile environment, ACICARE defines elementary components that represent the input modalities and composition components that specify how to use the input modalities. The ACICARE platform can be integrated with existing functionalities of the phone like the contact manager. For speech recognition, an embedded solution developed by Fonix Speech Inc., a commercial speech recognition system for isolated words, was used.

\section{B.6.4 MMI for navigation in complex mobile applications}

Zhang et al. presented a study comparing speech and GUI input for navigation in complex visualization on mobile devices [71]. The evaluation was carried out by using a mobile web browser (Visualizer) on an iPhone device with AT\&T Watson ASR as the network recognition system. Figure B.15 shows a common user interface using speech recognition capabilities. In the interface it can be seen that there is a "click to speak" button to activate this modality. The deployed page is modified to facilitate the navigation using a GUI. Six subjects participated in the evaluation. Four different tasks were done by each subject using speech and GUI input modalities. Zhang concludes that there is not much difference in effectiveness between speech input vs. GUI input, but this difference could increase depending on the task complexity. The team asserts that the speech input method is more efficient that the traditional method. Additinally, most of the users prefered the speech modality over GUI input.

\section{B.6.5 Parakeet}

Parakeet is a system for continuous speech recognition for mobile devices, developed at the Cavendish Laboratory in the University of Cambridge [62]. The application runs on a Linux mobile device (N800), and uses Pocketsphinx as the automatic speech recognition system [29], for U.S. and U.K English language models. In addition to the speech recognition engine, Parakeet has a correction interface that shows possible words ordered by probability. The user can select the correct word using the touch screen as shown Figure B.16. The blue line is the user trace of the selected words using the touch screen. Evaluation was done by two main experiments, a first one with four novice users completing 41 sentences indoors 


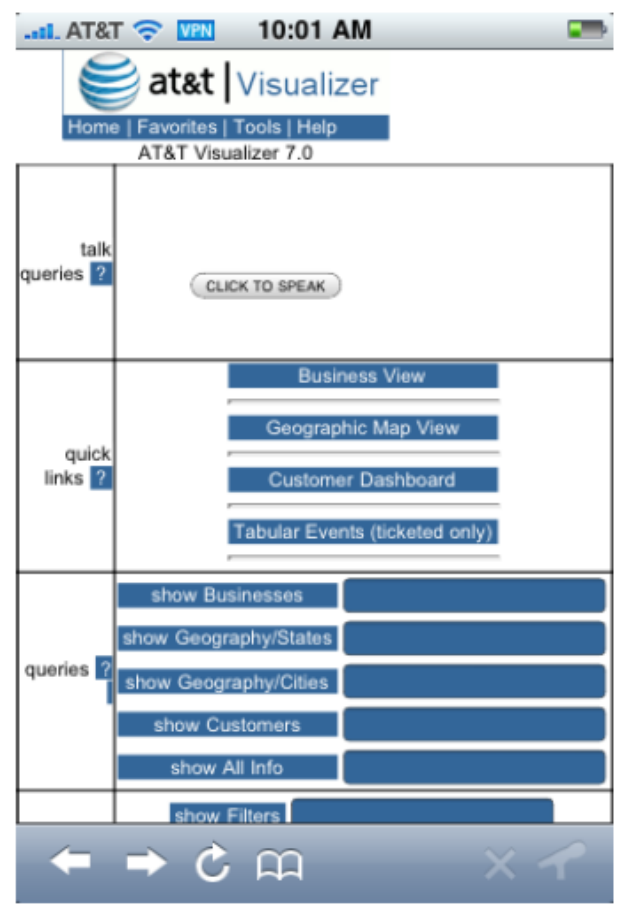

Figure B.15: ATEST multimodal interface [71]

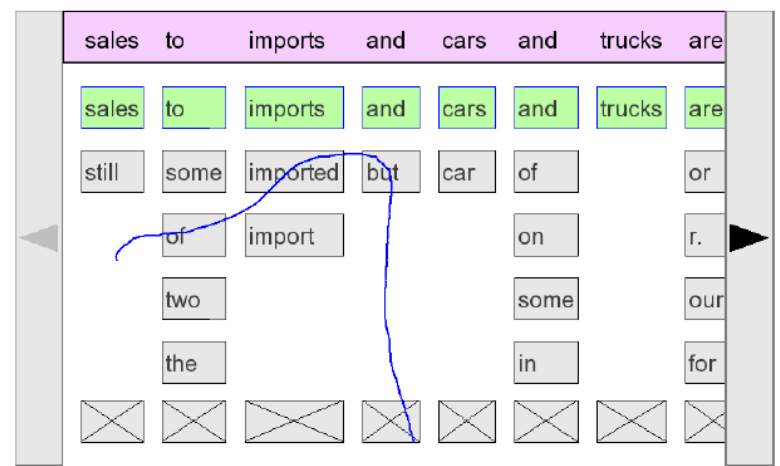

Figure B.16: Parakeet interface [62]

and 27 sentences outdoors, and a second one with one expert user, completing 313 sentences indoors and 309 sentences outdoors. In the first experiment the word error rates were $16.2 \%$ and $25.6 \%$, indoors and outdoors respectively. Using the correction interface the WERs were reduced to $1.2 \%$ and $2.2 \%$. For the expert user, the initial results were $8.4 \%$ and $14.8 \%$ and after correction $0.9 \%$ and $1.5 \%$ for indoors and outdoors respectively. The correction interface had a substantial impact on the WER. 


\section{Appendix C}

\section{Project Development}

To achieve the speech recognition navigation goal for the Borboleta system, a set of activities that addresses each one of the goals was defined. These include a review of existing software to select the speech recognition system, data collection of Brazilian utterances to train and test the system, design and development to define the system architecture and integration with the Borboleta, and testing to measure the system accuracy, its usability and acceptance level. This chapter describes these steps.

\section{C.1 Selection of the speech recognition system}

Current software for speech recognition has presented good accuracy results in large systems. In the last decade, mobile platforms have improved their capabilities to perform sophisticated tasks such as multimedia processing. Because of these improvements, speech recognition applications have been developed to target mobile platforms. The main goal in the software evaluation phase was to select a speech recognition system that could be integrated into the Borboleta system. The evaluation of the systems was done as follows.

The review process included speech recognition robustness and accuracy, software compatibility with mobile devices, software architecture, source code availability, license, and development community activity. Accuracy in speech recognition is important because executed commands depend on it. Therefore, the robustness evaluation was based on existing benchmarks related to the word error rate, response time and computer resource usage. Currently, large vocabulary automatic speech recognition systems present low word error rates because of computational capacity. Since the scope of this project is the use of speech recognition for commands on mobile devices, the vocabulary is smaller than for continuous recognition. While good performance was expected, the computational capacity and environmental factors were also expected to have an impact on the results.

Speech recognition software compatibility in mobile devices is related to the execution of the application on the device and the interaction or integration with the Borboleta system. There are assorted mobile platforms, which are developed in specific languages and with their own requirements. To find a tool that runs on any platform is difficult, so to reduce this problem, the software portability was restricted to the mobile devices used in the project and its development language. Borboleta is developed in Java for mobile devices (Java ME) although most of the devices are also $\mathrm{C}++$ enabled. Integration with Borboleta was evaluated on two possible interaction schemes. First, speech recognition as an external application running on the hand-held that communicates with Borboleta through any communication schema, and second, an internal module managed by the main system. The Borboleta system is open source software under the BSD license and the intention is to keep the same license for additional modules. By creating an open source system, an active development community can maintain sustainable software. 
Based on the above parameters, two speech recognition systems were evaluated, Pocketsphinx [29], an open-source project developed in the Carnegie Mellon University Language Technology Institute, and PocketSummit, developed by the MIT Computer Science and Artificial Intelligence Laboratory [24]. Although the two project are from high level research institutes and are based on robust systems, the first one was selected because of continuous development, an active community, open-source code, license compatibility and successful porting to other platforms like iPhone OS and Android. A complete description can be found in Section B.5.3. Once the ASR system was defined, the following steps included constructing acoustic and language models, and the definition of the communication schema and software architecture since Pocketsphinx is developed in $\mathrm{C} / \mathrm{C}++$ language and must interact with a Java ME system.

\section{C.2 Acoustic and language models development}

This section describes the used methodology to collect the audio samples and transcriptions to create the acoustic models. It also presents the acoustic and language models approaches defined for command speech recognition.

\section{C.2.1 Data collection}

To train the acoustic and language models and to test the system, a set of recordings and their word-level transcriptions was collected. The collection began by defining the vocabulary of voice commands that will be used during the application execution. After this definition of keywords, utterance collection and data preparation completed this phase of the project.

Keyword definition: Keywords are words that the system will recognize as commands. The keyword list is not composed of all the system functionalities, only the ones that need more than two user actions, such as button clicking or selecting a menu option, to be reached. Figure C.1 shows the navigation diagram for the Borboleta system. This diagram defines the current paths for any function in the system. Each edge value is defined as the cost for passing through the vertex (a window), that is, how many user actions will be required to access a specific function. Like a usability metric, a function cost can be defined to determine the commands, or keywords, the system will accept. Based on the diagram, the paths that are longer than two will be part of the keyword list. For example, if the user is in the Problems/Needs window and wants to go to the CID categories, the shortest path will be through Main menu, with a cost of three user actions. The fifteen commands on the keyword

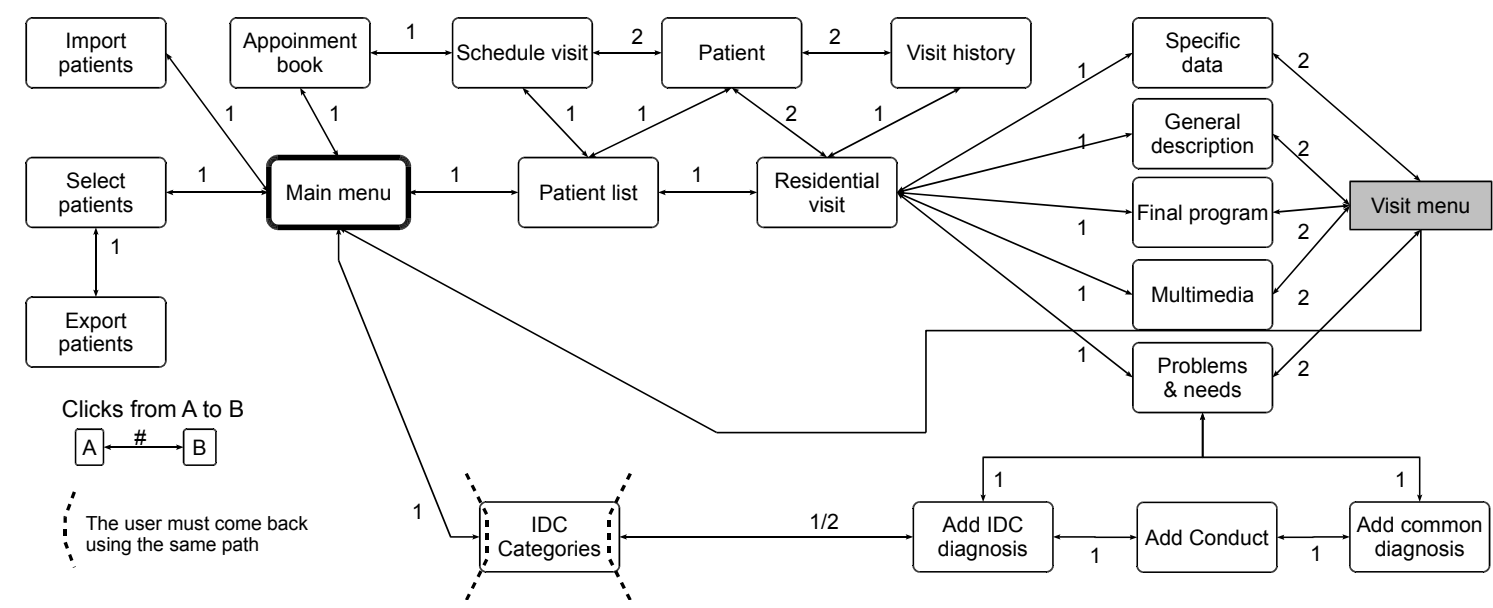

Figure C.1: Borboleta navigation diagram

list are shown in Table C.1. 
Table C.1: Keyword list for navigation on Borboleta

\begin{tabular}{|l|l|}
\hline Portuguese & English \\
\hline Menu inicial & Main menu \\
\hline Dados específicos & Specific data \\
\hline Caracterização geral & General description \\
\hline Problemas e necessidades & Problems and needs \\
\hline Programação final & Final program \\
\hline Encontros realizados & Visit history \\
\hline Encontro & Residential visit \\
\hline Novo encontro & New residential visit \\
\hline Paciente & Patient \\
\hline Agenda & Appointment book \\
\hline Agendar visita & Schedule visit \\
\hline Catálogo CID & International Disease Catalog (IDC) \\
\hline Sair & Exit \\
\hline Voltar & Back \\
\hline Fechar & Close \\
\hline
\end{tabular}

Utterance collection: To collect data that matches the expected application environment for training the system and to get workable results in the testing phase and later in a production system, the goal in this step was to emulate most of the environmental characteristics where the system will be used. First, the recordings were collected on devices similar to the ones used by professionals during medical visits. This reduces the effects of different microphones and it helped us to define the typical noise environment. Recordings were done in a reasonably quiet place, such as a room with normal environment noise and low external sounds. During the recording process, healthcare professionals held the hand-held device normally while directing their voice toward the device, and spoke naturally. Modifications in the way the user holds the device are not desirable because it affects the signal quality. At a consistent distance, the sound level can be used as an activation event for the speech recognition process. The system must recognize command utterances that are reproduced in a natural way and not just as isolated words. Figure C.2 shows the correct way to hold the device when recording data or using the system.

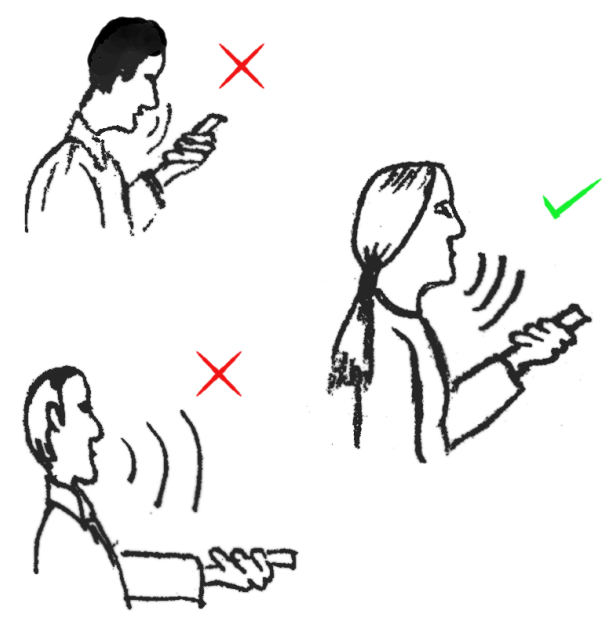

Figure C.2: Data collection method

The Borboleta healthcare team is composed of eight persons, 2 men and 6 women. With a keyword list of fifteen commands, and each team member recording five sets, 600 samples were 
collected to be used in training and testing phases. Samples were collected in WAV format using PCM encoding with an $16000 \mathrm{~Hz}$ sampling rate and 16-bit precision [11]. According to Huang [27], $16 \mathrm{kHz}$ sampling reduces errors by about $20 \%$ over a baseline of $8 \mathrm{kHz}$, which is typical for telephone speech. As new members are added to the Borboleta team, it is expected that recognition rates will be similar to those presented here because statistical modeling reduces dependence on particular users. This approach means that additional acoustic training, while beneficial, would not necessarily be required.

Data preparation: With the data collected, the next step was to prepare it for the speech recognition system. Faulty data, such as recordings with loud background noise, were removed. Then, the data was organized so that it could be accessed by the system. Most speech recognition systems require a dictionary that contains all the words the system will support, a filler file of non-speech sounds, a file path list that the system uses to find data, a file containing the available unit sounds whether words or phones, and the transcriptions. Construction of the dictionary and the phone file uses the Speech Assessment Methods Phonetic Alphabet (SAMPA) [40]. SAMPA for Portuguese was also used in the FalaBrasil project [44]. The data was separated into a training set to create the acoustic models and a test set for evaluating system accuracy.

\section{C.2.2 Training phase}

$70 \%$ of the collected data was used to train the system, generating the acoustic models for the different modeling approaches: isolated commands, whole-word models, and phonebased recognition. The language model (LM) defines the structure of the language, that is, defines how the commands must be formatted to be accepted by the system. For the command speech recognition system a finite grammar structure (FSG) language was used, based on the navigation diagram. Figure C.3 is an example of language model, in this case word1, word2 or word3 are accepted initially, but word4 and word5 are accepted if they are preceded by word1.

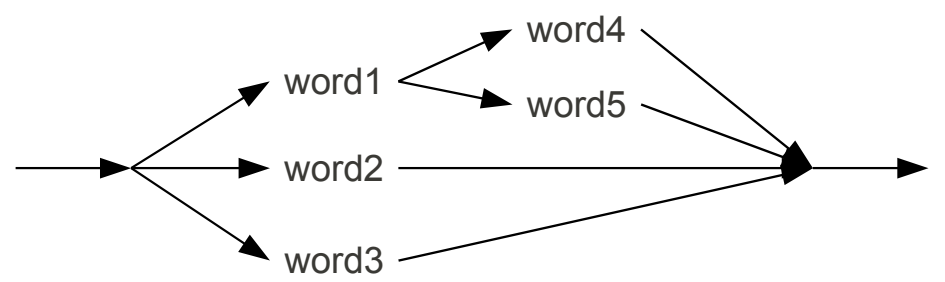

Figure C.3: Language model example (JSGF)

\section{Approaches for command recognition}

In order to define which speech recognition approach has the required high accuracy level, four command word modeling and language models combinations were tested. According to SphinxTrain requirements, two dictionaries were created with their own phoneme set, and two language models were built. Table C.2 shows the structure of the sphinx dictionary and its corresponding phone file. A dicX file contains the supported commands with their transcriptions. wordX is a supported word or command in the system, and a $p h X$ represents a label for an acoustic model. While this typically represents a phone, this work uses both phone and word-level labels in this list.

\section{Approaches}


Table C.2: Dictionary example

\begin{tabular}{|c|c|}
\hline $\begin{array}{l}\text { Dictionary } \\
\text { dicX }\end{array}$ & $\begin{array}{c}\text { Acoustic modeling } \\
\text { phone } X\end{array}$ \\
\hline 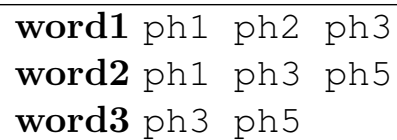 & $\begin{array}{l}\text { ph1 } \\
\text { ph2 } \\
\text { ph3 }\end{array}$ \\
\hline & $\begin{array}{l}\cdots \\
\text { ph5 }\end{array}$ \\
\hline
\end{tabular}

1. Whole-word / Composed command: The whole-word term indicates that words in the dictionary are modeled without being decomposed in phones. The acoustic model units are words rather than phones. The commands in this approach are constituted of a string of words. For example the command NOVO ENCONTRO has two words in the dictionary, and in the language model there must be a constraint to filter word chains that do not match this command chain. Table C.3 shows an example of this approach, where the language is a set of three words, which are modeled as whole words, and the language model accepts the commands word1 word3 or just word2 or word3.

Table C.3: Command recognition - Approach 1 example

\begin{tabular}{|l|c|l|}
\hline Dictionary (dict1) & Phone (phone1) & LM (lm1) \\
\hline word1 word1 & word1 & (word1 and word3) or \\
word2 word2 & word2 & (word2) or (word3) \\
word3 word3 & word3 & \\
\hline
\end{tabular}

2. Phoneme / Composed command: In the phoneme modeling case, whole words in the dictionary are decomposed into phones, and a phone file contains all supported phones (including silence). The word decomposition was done using SAMPA conventions [40]. For language modeling, the same structure of the first approach was used. This is shown in Table C.4. The full phone decomposition of keywords is in Table F.1 in Appendix F.

Table C.4: Command recognition - Approach 2 example

\begin{tabular}{|l|c|c|}
\hline Dictionary (dict2) & Phone (phone2) & LM (lm1) \\
\hline word1 ph1 ph2 ph3 & ph1 & Same as \\
word2 ph1 ph4 & ph2 & Approach 1 \\
word3 ph3 ph4 ph5 & $\ldots$ & \\
& ph5 & \\
\hline
\end{tabular}

3. Whole-command / whole word: In this case, the dictionary contains the commands as a simple string, that is, it composed of the supported words. The language model for this approach contains only the supported commands. In Table C.5 the command word1_word2 contains word1 and word2 as subunits. In the language model two commands are supported and other sequences will be rejected.

4. Whole command / Phonemes: Similar to approach 3, the dictionary contains the commands as string chain, but the decomposition is done at the phone level. The same language model is used as in approach 3. Table C.6 shows this approach.

Initial testing was carried out on the speech recognition software running on a desktop computer. Running the experiments on a desktop computer did not affect the accuracy results 
Table C.5: Command recognition - Approach 3 example

\begin{tabular}{|l|c|c|}
\hline Dictionary (dict3) & Phone (phone1) & LM (lm2) \\
\hline $\begin{array}{l}\text { word1_word2 word1 word2 } \\
\text { word3_word4 word3 word3 }\end{array}$ & Approach 1 & $\begin{array}{c}\text { (word1_word2) or } \\
\text { (word3_word4) }\end{array}$ \\
\hline
\end{tabular}

Table C.6: Command recognition - Approach 4 example

\begin{tabular}{|l|lll|l|}
\hline Dictionary (dict4) & Phone (phone2) & LM (lm2) \\
\hline $\begin{array}{l}\text { word1_word2 ph1 ph2 ph3 ph1 ph4 } \\
\text { word3_word4 ph3 ph4 ph5 ph5 ph1 ph2 }\end{array}$ & Approach 2 & Approach 3 \\
\hline
\end{tabular}

because the training and testing data was collected with mobile devices and the software was limited to run on mobile devices. Recognition was performed on the remaining $30 \%$ of the collected data, using desktop simulations for preliminary results. The preliminary testing was used to select the modeling approach used in the final system. Results are shown in Chapter D

\section{C.3 Software design and development}

The Borboleta software is developed in Java for mobile devices (Java ME). This platform guarantees software compatibility across a broad range of handhelds. However, there are different Java Virtual Machine (JVM) implementations, each one compatible with a specific mobile platform. Currently, specific implementations do not have the same functionalities. For multimedia processing, there are only a few implementations that offer media capture functionality; thus, not all devices are capable of performing speech recognition. $\mathrm{C}++$ is available in many devices and has the advantage that most mobile platforms have been developed in this language, so access to resources is faster. Speech recognition algorithms could run faster than on a virtual machine. However $\mathrm{C}++$ implementations are commonly platform-dependent, reducing the application compatibility range.

The software design and development phase was approached from two sides, porting the speech recognition software to a mobile device platform, and integrating the software with Borboleta. Porting the software was carried out by evaluating the language compatibility, software robustness and accuracy. Language compatibility is related to implementation options for the different speech recognition phases, like media capturing, signal processing and word searching. Evaluating the software robustness and accuracy allows assessment of the application development level and comparisons with previous results on mobile devices. Software integration with Borboleta refers to the communication schema between the two applications. The communication depends directly on the selected platform and its available resources.

Pocketsphinx is part of CMUSphinx toolkit, a set of tools for speech recognition processing. Also, the toolkit provides Sphinx3, a speech recognition decoder for researching written in C, Sphinxbase, a support library for Pocketsphinx and Sphinx3, Sphinx4 a recognizer written in Java, SphinxTrain, a tool for acoustic modeling training, and CMUCLMTK, for language modeling. Since Pocketsphinx is written in C, the first target was to port the system to the Symbian platform, second, an intercommunication schema $\mathrm{C} / \mathrm{C}++$ and Java was implemented, and finally, a module that supports the multimodal interaction in Borboleta was developed.

The software development process was carried out using an agile software methodology. Small targets were defined to fulfill more complex tasks. Initially, all the efforts were concentrated in the Pocketsphinx porting process, language compatibility using Open $\mathrm{C} / \mathrm{C}++$ 
and Symbian configuration for compilation for the emulator and for devices. With a basic port, the next target was the intercommunication schema, where a socket approach was implemented on both sides, $\mathrm{C} / \mathrm{C}++$ and Java. A third step was the implementation of the multimodal module that lets the user have access to the system using his/her voice. Having a reduced version of the full system, all the submodules were improved. The Mobile Speech Recognition Services (MSRS), as the $\mathrm{C} / \mathrm{C}++$ side was called, was improved by adding support to multiple application clients. The communication protocol was completely defined, and in Borboleta, support for all the commands was implemented.

The remainder of the section describes the development environment, the Pocketsphinx porting process, the MSRS architecture, the $\mathrm{C}++/$ Java ME interaction, and the multimodal implementation approach

\section{C.3.1 Development environment}

Since different languages were involved during the project development, several development environments were used. Pocketsphinx can be built on $\left(^{*}\right)$ nix and Windows systems targeting different platform (Linux, iPhone, Android, Windows). Until recently, Symbian has been developed on Windows machines, but there have been recent efforts to build Symbian applications on Linux systems based on Wine and the GnuPoc project, ${ }^{1}$ but not all capabilities are available yet. Therefore, Carbide, a Windows-compatible integrated development environment (IDE), was used to build the Pocketsphinx port for Symbian. Although Borboleta can be built in Linux systems, the Windows version of Netbeans was used in order to facilitate the development and testing of both sides. Finally, QtCreator, a Qt/C++ IDE was used to create the MSRS user interface and to build graphical interfaces compatible with Symbian. More detailed descriptions of the IDEs are included in the Appendix G.

\section{C.3.2 Pocketsphinx porting}

In order to run Pocketsphinx on Symbian devices, it must be configured and adapted to the target environment and its requirements. This process is commonly called porting, and it is composed of a task set that tries to cover most of the aspects of the code migration during the process. The Pocketsphinx porting done here was based on Mark Wilcox's book, Porting to the Symbian Platform [67], and the advice from Nokia forum community. ${ }^{2}$ In his book, Wilcox defines a porting process with ten flexible phases, including project selection, code analysis, re-architecting the software, development environment set up, integration with the Symbian build system, compilation, fix problems, run and test, debug, and re-integration with the original code. Figure C.4 shows the porting process flow. Below is presented each phase and how they were applied in the project.

\section{Project selection}

Choosing software to be ported covers the evaluation of technical aspects, like the used language, as well as intrinsic project features, like community activity or project status. Section C.1 described how the speech recognition software was selected for this work. In summary, Pocketsphinx was selected due to the continuously active development and the several communication channels, like forums, mailing lists, issue tracker and a IRC channel, to get support from authors and other communty members. Also, Pocketsphinx had been previously ported to other platforms with similar hardware characteristics, and a prior porting for Symbian had been made. Finally, Pocketsphinx has been developed under a BSD-like

\footnotetext{
${ }^{1}$ http://www.martin.st/symbian

${ }^{2}$ http://www.forum.nokia.com
} 


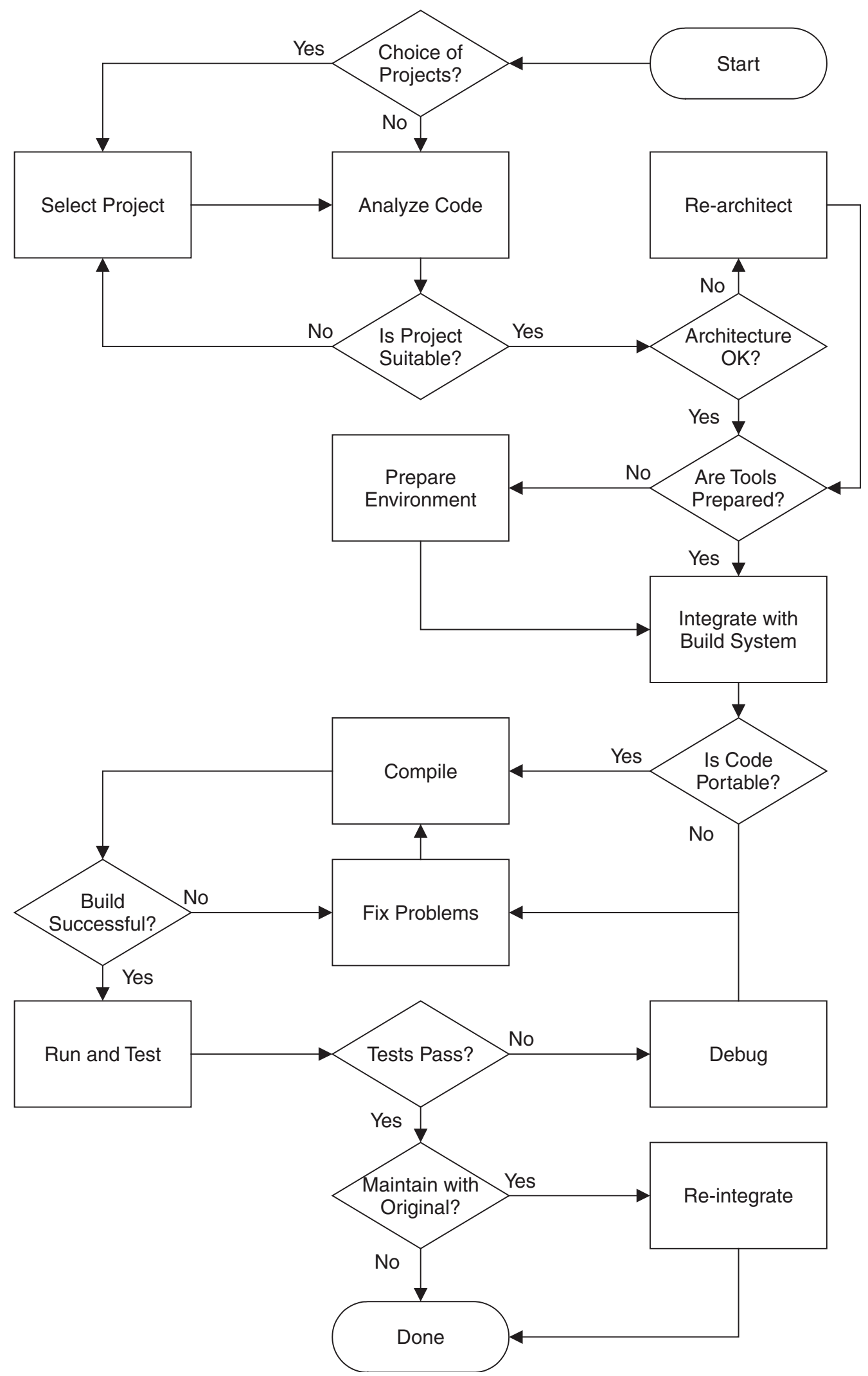

Figure C.4: Porting process [67] 
license compatible with the Borboleta license, and ANSI C standard has been used, which is highly compatible with Symbian because of the OpenC/C++ and POSIX libraries.

\section{Code analysis}

The aim of the code analysis step is to understand the project organization, the external libraries dependences, and the possible places where modifications must be done. Pocketsphinx is structured in two subprojects. First the Sphinxbase contains the basic functionalities that are shared between the CMUSphinx projects, the trainers and the Sphinx II, III and Pocketsphinx and utilities for acoustic processing. It has a source and a header directory and specific folders for the different targets, and uses Automake, Autoconf and Libtool as building tools. The Pocketsphinx decoder is similarly organized to the Sphinxbase, with a folder for sources, another for includes, and specific folders to implement functions for given targets. Most of the software was written in standard C, including the hardware access routines, reducing the needed modifications when migrating the application. Most of the code is self-documented, and there is also documentation for installation, training and decoding.

\section{Development environment set up}

This step is related to the development tools configuration for the Symbian platform. A recommended machine for Symbian development is one that has Microsoft Windows 2000 service pack 3 or higher, at least $512 \mathrm{Mb}$ of RAM, a x 86 processor of $1 \mathrm{Ghz}$ or faster, minimum $1.5 \mathrm{~Gb}$ of free disk space, and a 16bit color screen. It must have Java JRE and ActivePerl 5.6.1 installed, and administration rights. The development environment for the project is fully described in Section C.3.1 and Appendix G.

\section{Re-architecting and porting}

During the code analysis, the code structure, the basic software architecture, and the possible places where code must be added or modified were evaluated. Now, in this step, a deeper evaluation was done in order to port the software to the target platform. Having a separation between the logic model and the user interface in the software brings a series of advantages to the software development, like the portability of a logic model through different graphical interfaces, or to be able to test the logic model independently from any graphical interface. Since Pocketsphinx is composed of a set of decoding tools to be used on different applications, it does not include graphical interfaces, so there is no division between model and presentation. This principle was taken into account during the development of the user interface of the system using Qt for the presentation layer and the Pocketsphinx library as a part of the logic model.

Since an early porting had been done, it was taken as base to start the Pocketsphinx porting. Evaluating the porting strategy with other targets, it was identified that preprocessor directives were used to select code sections during the compilation, and there were specific files for platform-dependent functions. At the final port fifteen files were modified and created, with ten for building configuration, which are explained in the integration with the Symbian build system section below, one header, and four C files. Previously, a native implementation for accessing the recording device on phone had been developed, it was slightly modified to support compilation for emulator and devices without affecting other targets.

\section{Integration with the Symbian build system}

With the code changes done, the next step was to integrate the software with the Symbian build system. This build schema uses the same paradigms of other automatic build systems, 
like the autogeneration of makefiles for different platform architectures, by giving an input configuration file. The configuration file for the Symbian build system are the bld.inf and the $M M P$ files. The first defines the target platforms to be compiled and the projects that must be included. The second file (MMP) is a project configuration file, where the parameters to compile the project are configured. The binary name (TARGET), the binary type (TARGETTYPE - dll, exe, stddll or stdexe), the unique identifier for the target type, subtype and global identifier (UID), the device capabilities the software uses (CAPABILITY), the own headers (USERINCLUDE), the system headers (SYSTEMINCLUDE), the folder where the sources are located (SOURCEPATH), the source files to be compiled (SOURCE), memory constrains (EPOCSTACKSIZE), and library dependences (LIBRARY), among others, are set in the project configuration file. To build the application for ARM targets, a package configuration file (.pkg) is needed to map the built files and the destination in the devices, as well as the information about library dependences. Appendix H contains the MMP files for Sphinxbase and Pocketsphinx.

\section{Compiling}

In the compiling phase the binary files were built by first compiling the basic functions and then adding the more complex tasks. In the project, the Sphinxbase was configured and compiled initially, then the Pocketsphinx library was obtained, and finally two executables were built, the pocketsphinx_batch and the pocketsphinx_continuous which are command line applications to test the decoder in batch with audio file lists or with live audio input. Build errors are due to compiler and linker differences, missing dependences, mixing $\mathrm{C}$ and $\mathrm{C}++$, or the function exports from DLLs. In Symbian, these exports must be done explicitly by including EXPORT_C for definitions and IMPORT_C for declarations. In the new STDDLL and STDEXE target types, these definitions are not required. The Sphinxbase and Pocketsphinx configurations were updated to use the STDDLL and STDEXE targets for libraries and executables to reduce the number of modifications in the source.

\section{Fixing problems}

Once the application is running on the new target, some execution aspects must be evaluated, especially memory consumption in mobile devices, CPU usage which affects battery life, and the graphical interface, which must be adapted to small screens with lower resolution levels. For Pocketsphinx execution the memory parameters weere increased in order to load the acoustic and language models ( $80 \mathrm{~Kb}$ to the stack, and $16 \mathrm{Mb}$ to the heap). The initialization parameters were changed based on the optimization guide from the CMUSphinx site, ${ }^{3}$ to get better performance results with an acceptable accuracy level.

\section{Running and testing}

Ported Symbian applications must be tested in at least two scenarios, the emulator and the real device. Emulator testing ensures that standard procedures are correctly executed without deployment in a real device, reducing the testing time. For hardware-access functions, directly testing on the device is mandatory. A basic set of features to be tested were defined: the decoder initialization, language and acoustic model load, correct capture of utterance and its respective processing, and the speech recognition for continuous and isolated words. Software performance and parameter settings were also checked.

\footnotetext{
${ }^{3}$ http://cmusphinx.sourceforge.net/wiki/pocketsphinxhandhelds
} 


\section{Debugging}

Debugging Symbian applications is similar to normal debugging in other platforms, except for the lack of the stdout and stderror by default in the execution environment, which are essential during the debbuging process to trace function errors and variable values. Pocketsphinx debugging wa done using the Carbide.c ++ debbuging tool.

\section{Re-integrating the code}

Re-integrating the code refers to the merge of the Pocketsphinx modification to the development trunk. An evaluation of the merge must be done by checking that ancient targets still compile and the modification does not affect the execution of the application in such targets. The Pocketsphinx merge was done with the support of the CMUSphinx developers ensuring a correct execution on other platforms.

\section{C.3.3 Mobile Speech Recognition Service (MSRS) architecture}

A speech recognition service for mobile devices was developed to verify whether the option of speech recogntion for input improves software usability. This service is composed of a decoding module, a user interface and an inter-application interaction schema. Based on the three different ASR implementations for mobile devices, embedded, network and distributed speech recognition (See Section B.5.2), the option that allows for both command recognition and offline execution is embedded recognition (ETS). Thus, the MSRS was designed as an rich embedded application, where all functionalities are executed by the device and no external connection is required.

In addition to command recognition and offline execution, there were five system architecture requirements. First the system must run on a Symbian environment because most of the devices used in the Borboleta project are Symbian-based. Second, it must be able to access the Pocketsphinx API to make speech recognition features available. Third, the system must provide a basic user interface for editing configuration. Fourth, the system must provide an interaction mechanism between applications, including Java and $\mathrm{C}++$ based programs. Fifth, a Java client that interacts with the speech recognition system must be developed and integrated with the Borboleta application.

Giving these requirements, SpeechMe, the speech recognition system developed here, is divided in two subsystems, the $\mathrm{C}++$ side that makes speech recognition available, and the Java side, that uses the service through a socket connection. The $\mathrm{C}++$ side is a $\mathrm{Qt}$ application composed of a graphical user interface (MSRS_GUI), the Pocketsphinx decoder, a $\mathrm{C}++$ layer that accesses the Pocketsphinx API and provides the speech recognition features (MSRS_NATIVE), and a Qt-based communication module that interacts with external applications. The Java side is an application module integrated with a multimodal subsystem in the Borboleta application. The Java module coordinates the recognition for the Java application for both isolated and continuous recognition. Based on the Symbian operating system architecture, SpeechMe is situated in the higher layers of the platform, but uses functionalities from different layer levels. Pocketsphinx and MSRS_NATIVE directly access the Open $\mathrm{C} / \mathrm{C}++$ and PIPS ${ }^{4}$ libraries. Qt uses Open $\mathrm{C} / \mathrm{C}++$ and PIPS libraries, but also access the Symbian C++ API, a level lower than the others. On the Java side, the communication schema uses the Java API provided by the platform. Figure C.5 show the SpeechMe deployment on a Symbian system.

The general architecture of the system is shown in Figure C.6, with the two main sections, the $\mathrm{C}++$ side and the Java side. The architecture presents modules of both subsystems, their interactions and their encapsulation levels. A module interacts directly with its parent, that

\footnotetext{
${ }^{4}$ PIPS is an acronym term for P.I.P.S Is POSIX for Symbian OS
} 


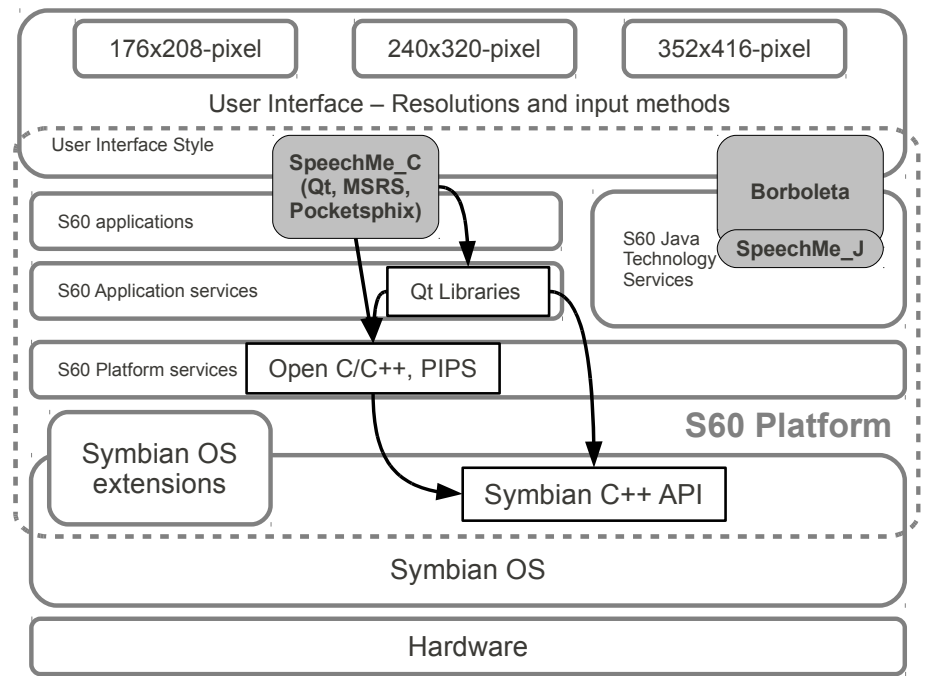

Figure C.5: Mobile Speech Recognition Service on Symbian architecture

wraps it, or with its children, that it wraps. In the architecture, the concept of a multimodal form is introduced. A multimodal form is a normal graphical form from the presentation layer that supports extra input modalities, such as speech recognition, gestures, and face recognition. Here, the multimodal form includes a button to activate speech recognition. When it is activated, the form asks the SpeechMe module to use the speech recognition service. The SpeechMe module requests the service through a socket connection to the SpeechRemote module. Since SpeechRemote belongs to the $\mathrm{C}++$ side, it processes the request and calls the MSRS to start recognition. Once the recognition is complete, the phrase is sent back to the SpeechMe module in Java using SpeechRemote again. When the phrase is in the Java side, it can be used to execute a command in the application, like navigate to another window, or save it in the application, for example, as a medical note. The activation button included in the multimodal form has been used previously by Zhang et al. [71]. Having a button to activate the service reduces the use of hardware and increases the phone battery life. During the information flow, from the recognition request until receiving the phrase, messages are interchanged across the $\mathrm{C}++$ and Java sides. These messages report the decoding status to the application clients, so that the application can show the user the current phase. For example, the user can be notified when to start talking, or the application could disable speech recognition in case of a decoder error.

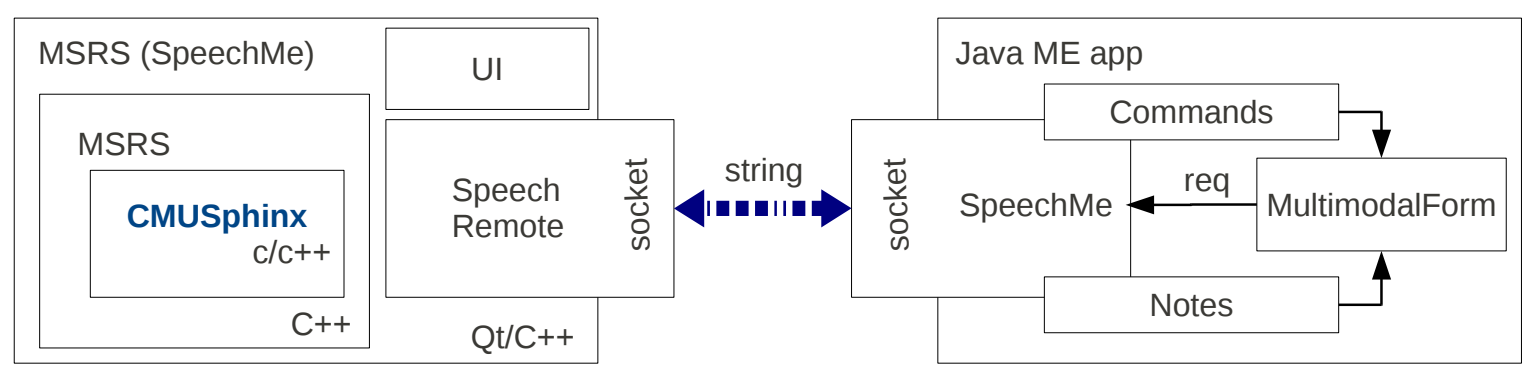

Figure C.6: Mobile Speech Recognition Service - General Architecture

Sections C.3.4 and C.3.5 presents the design and implementation of the C++ and Java system sides. Section C.3.6 shows the development of the communication protocol in the interaction of both sides. 


\section{C.3.4 MSRS native (open $\mathrm{C} / \mathrm{C}++$ )}

The speech recognition system was conceived as a composition of multiple modules in a layer scale. At the lowest level is the Pocketsphinx decoder. Although Pocketsphinx was developed mainly in C, some features are coded in Python and Perl. Pocketsphinx directly accesses the OpenC/C++ and POSIX libraries that Symbian provides. The decoder is structured as a set of functionalities used for speech recognition, with signal processing functions, procedures for feature extraction, pattern recognition and word search, microphone initialization, language configuration, and utterance decoding. This set constitutes the API for speech recognition. Currently, Pocketsphinx does not include a formal procedure to perform recognition from source initialization to utterance decoding, but it comes with programs for live and batch decoding which were used to fully implement a recognition procedure in this work. This implementation is located in an upper level (MSRS). The MSRS is implemented as a class (Msrs) that wraps the decoder and performs functions calls to complete speech recognition in a specific method. To avoid the user interface locking during decoding, the Msrs class is wrapped in a QThread class with its own event loop, and the method calls are done through the Qt message communication (Signal/Slot). This methodology is commonly used in inter-thread communcation to execute functions independently.

A crucial feature in the recognition process is to constantly report the decoding status to the clients. The Msrs class maintains a list of possible decoding status as public static int values that are sent to the client each time the status changes. The class also provides methods for decoder configuration and initialization. To report the decoding status as well as the decoded utterance, an Observer Pattern [22] was implemented. There is a parent class (Subject) that maintains the observer list and has the methods for adding and removing observers. The Msrs class extends the Subject class to notify the observers of events. The status notification process could be executed during the configuration, initialization or recognition procedures. Appendix I lists all the possible statuses. All the Msrs observers must implement the Observer interface. There are two observer types, the permanent observers, that always receive status notifications, and transient observers, that are notified based on recognition requests. If multiple clients are asking for the service, only the first is responded to and the system remains busy until the current client receives its decoding. Then the system is free for new requests. No multiple transient clients are allowed in order to avoid applications with different goals using the same sentence. A permanent client is the SpeechMe class, the base class in the system, and will be described later in this section.

The local clients are the $\mathrm{C}++$ classes that access the MSRS to configure and test the system. Local clients are in the highest level in the architecture. They are composed of a configuration and a local recognition test user interfaces. The configuration UI (Figure C.7(a)) was developed in Qt. Here a configuration file is selected, and the server port for external connections is configured. The configuration file is passed to the decoder during the intialization. This file contains the location of the acoustic model, the language model, the dictionary and other parameters to configure the decoder. For this project, there is a main folder which has a subfolder and two files. The language model file can be in Java speech grammar format, ${ }^{5}$ or in Pocketsphinx format for statistical ARPA language models. ${ }^{6}$ The language is set in the configuration file with the parameter -jsgf or - lm respectively and the path to the file. The second file is the dictionary, a text file containing all the supported words which is set in the configuration file with the parameter -dict plus the file path. The subfolder (-hmm parameter) contains the acoustic model files previosly generated on a desktop system using the SphinxTrain application. To configure the socket server a port number must be selected. The Load button is used to configure and initialize the decoder.

The local recognition test UI is used to test the system without external clients. Speech-

\footnotetext{
${ }^{5}$ http://www.w3.org/TR/jsgf

${ }^{6}$ http://cmusphinx.sourceforge.net/wiki/tutoriallm
} 
Pad, the test widget, is composed of a multiline text box, where sentences are shown, and two buttons for isolated and continuous recognition. In isolated recognition mode, the system captures utterances while there are not silence spaces. At the first silence the decoder stops and returns the decoded sentence. By clicking the continuous button the system captures utterances normally. If there is a silence, the sentence is sent to the client, but the decoder starts capturing a new utterance for the same client. To stop continuous recognition the same button must be clicked again. Figure C.7(b) shows the SpeechPad UI.

An additional UI is the SpeechWeb widget. This UI was developed to test speech recognition in other fields. The widget consists of an embedded Qt web browser (QWebKit) that uses decoded sentences to send a query to the Google search engine and show its results. This is also a local client, but it is transient because it is only notified while the widget is shown. Figure C.7(c) presents the SpeechWeb UI.

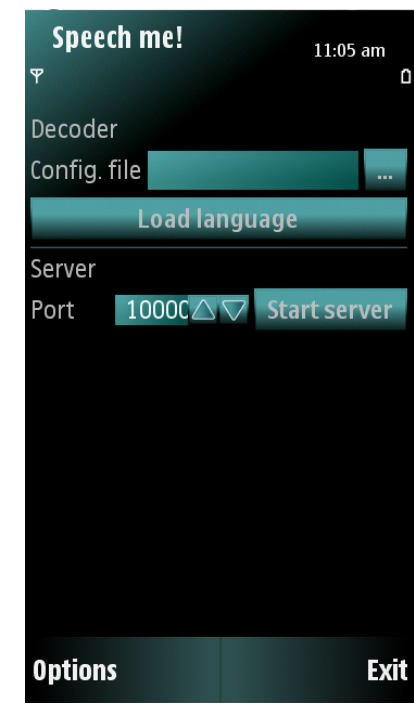

(a) SpeechMe Configuration

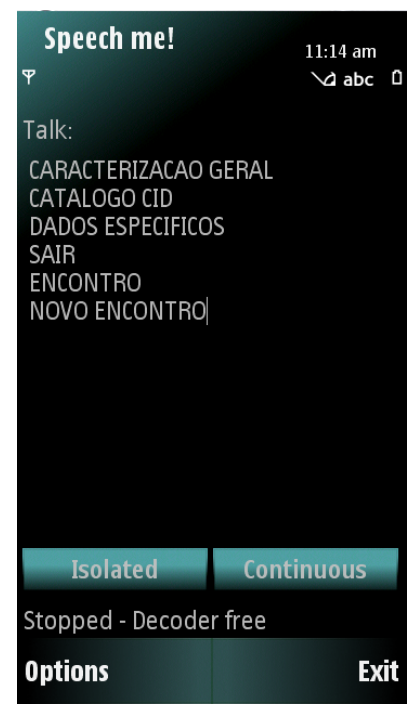

(b) SpeechPad

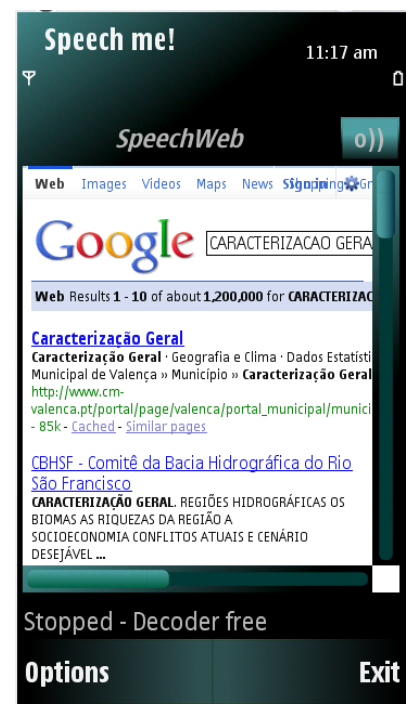

(c) SpeechWeb

Figure C.7: SpeechMe GUI - Qt/C++

Another component is the SpeechRemote module, which is responsible for communication with external applications. This module is composed of the SpeechRemote class that has a socket server listening for new connections and the RemoteClient class that represents the external application in the system. RemoteClient is a transient observer for the MSRS, and it only receives notifications and sentences if it is the current transient client in the MSRS queue. The full specification of the external communication is presented in the Section C.3.6.

Finally, the SpeechMe class is a base class that coordinates all the operations in the system. This class has an instance of the MSRS, and is a permanent client of it. It has a QMainWindow UI that is used to show the three widgets presented before, which are accessed through a simple menu. In the Qt-mobile version, it is shown in the lower part of the screen. There is also a status bar that shows the decoder status. SpeechMe also has an instance of the SpeechRemote system. Being a central class, SpeechMe processes all the local and external requests, and redirects the call to the specific class. Configuration, a plain class, is used to keep, independently, the configuration parameters to be loaded in the Configuration UI or saved after any modification. The SpeechMe C++ side uses a Strategy Pattern to structure the system in a way that procedures are encapsulated depending on their functionalities or objectives, trying to reduce the maintaining cost and allowing easier understanding for future developers. Figure C. 8 shows a basic class diagram of the SpeechMe design on the $\mathrm{C}++$ side. 


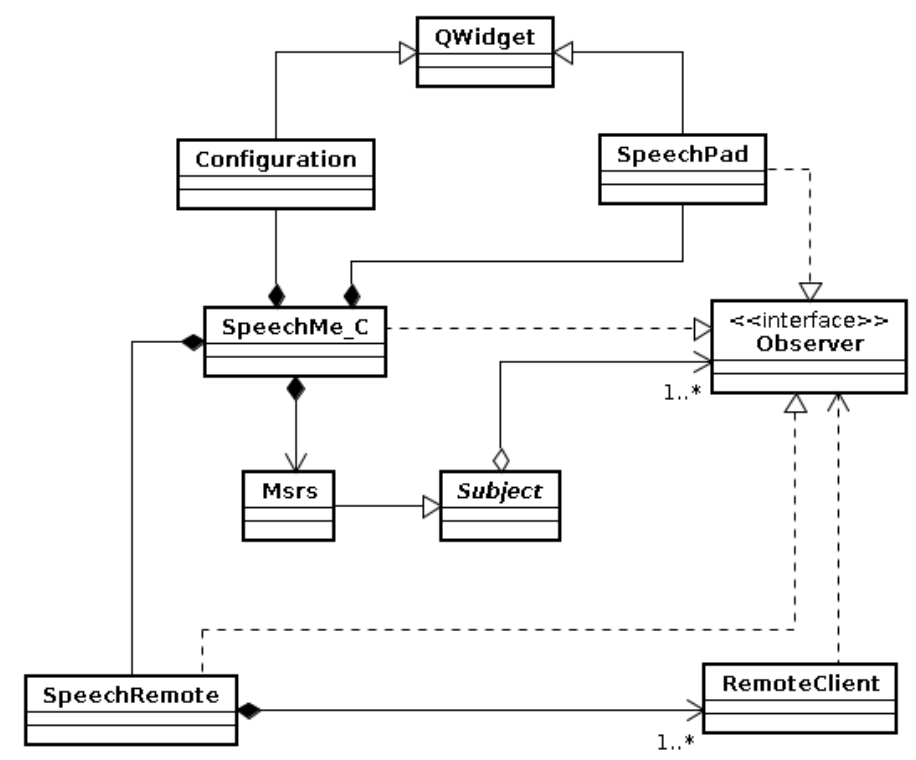

Figure C.8: Speech Me architecture $-\mathrm{C}++$ side

\section{C.3.5 MSRS JavaMe side}

The MSRS on the Java side is the module responsible for getting recognition requests, sending them to the $\mathrm{C}++$ side through a socket connection, and listening for system responses, either for decoding status or the decoded sentence. This module was designed as a Model/View/Controller compound pattern [22], where the model is the SpeechMe class that controls the communication, sends requests and and receives responses. The set of views are the ones that show the user the state of the decoding and allow the user to set the configuration parameters for this module. SpeechController is the controller class that coordinates both the model and the views. The remainder of this section explains how each component in the Java side MSRS module, the $\mathrm{C}++/$ Java interaction schema, and the multimodal approach to execute navigation commands was implemented.

\section{SpeechMe - Model}

The SpeechMe class represents the place where recognition requests are sent to a speech recognition system, and where the responses are processed and specific methods are called depending on the processing result. The class is composed mainly of a SocketConnection instance with its respective input and output streams, a reference to an object that implements the ModelController interface, which is used to interact with the controller, and an InReader class instance. This class is constantly listening for responses from the MSRS in its own thread, and sends the responses to the SpeechMe class to be processed. The SpeechMe class is implemented using the Singleton pattern [22] to guarantee that just one instance of this class provides the speech recognition capabilities in the Java ME application. The proposed communication protocol is implemented in this class as well as the activation and deactivation of the speech recognition functionalities (activateService method). The communication protocol and how is it implemented in the SpeechMe class is explained in more detail later.

\section{MultimodalForm - View}

While speech recognition is active, various Forms could access the service. To use the service, the Form must provide a button to request recognition. There are two options to implement this. First, each Form that wants to provide the service creates the button, 
captures the event from the user, requests the service from the speech controller and captures, processes and shows the decoding status messages. The other option is to have a single implementation of these functionalities and use it in each Form that wants to provide the service. Since all the forms in the applications extend the Form class from LWUIT, $^{7}$ what is proposed is to extend the Form class through a MultimodalForm that has the same common Form services, but that also captures multimodal events. Then, all the forms extend the MultimodalForm giving automatic support to speech recognition reducing the number of modifications in the code.

The MultimodalForm was designed to interact with the user in a multimodal way by providing alternative input and output methods. In this project, the Multimodal form presents the user with a simple button in the top right corner on the screen in the title bar to activate speech recognition to execute navigation commands. The button is easily accessible and does not use extra space in the screen (Figure C.9). When a MultimodalForm is shown, it is registered in the speech controller as a current client for speech recognition services. If the service is enabled and the button is clicked, the Form asks the speech controller to start recognition to execute a navigation command. Since the Form is the current client, the controller shows the user a status dialog box over the form (SpeechDialog class). In this way, the controller keeps the user updated about the recognition phases. Figure C.10 shows how the user is notified about the recognition progress, from left to right. When the button is clicked, the system opens the microphone and the dialog shows a wait signal. When the decoder is ready to listen to the user, a cloud dialog is shown indicating that the user can start speaking. When the utterance is captured, a cloud dialog with lines is presented. If decoding is successful the dialog is closed and the command is executed, but if there is an error, a fail icon is presented to the user. The error can be seen in the speech configuration view.

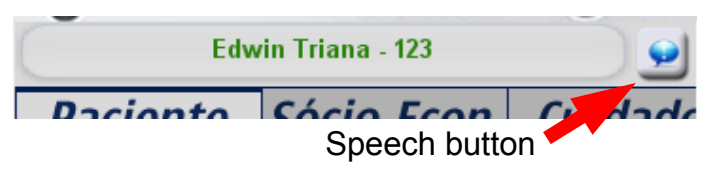

Figure C.9: Speech button

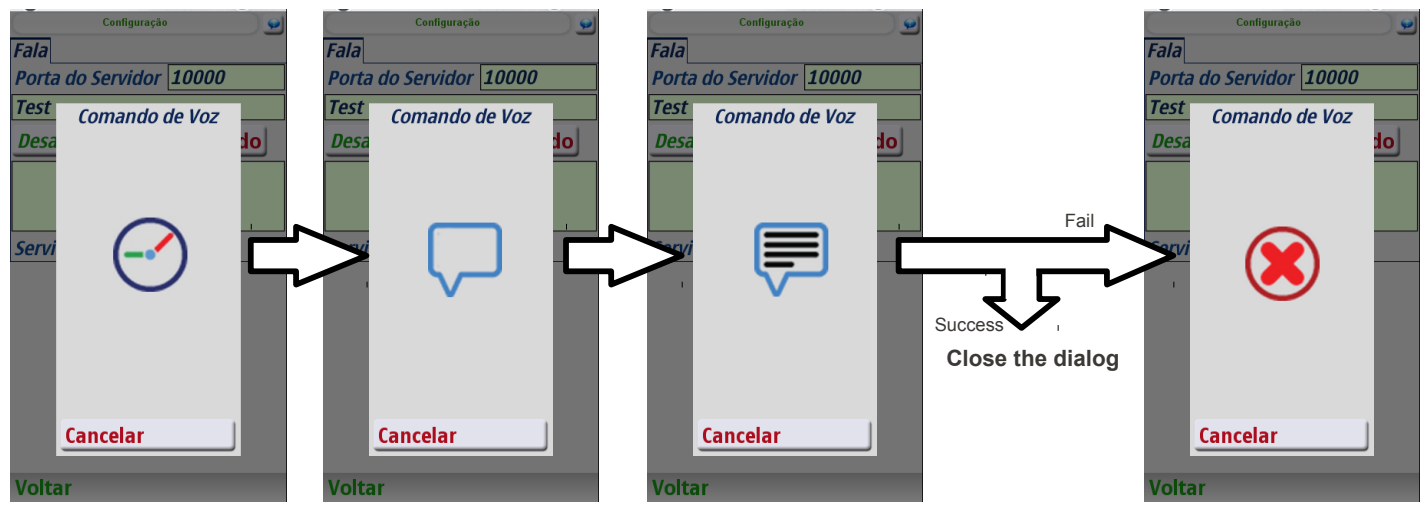

Figure C.10: SpeechDialog view

\footnotetext{
${ }^{7}$ Graphical components to build user interfaces in Java ME. See https://lwuit.dev.java.net
} 


\section{SpeechConfigurationView - View}

SpeechConfigurationView is a tab view embedded in the main configuration form (ConfigurationForm). The ConfigurationForm extends the MultimodalForm class that enables the speech recognition in the application forms. Figure C.11 shows the configuration view for speech recognition. The left side shows a screen with recognition deactivated and the right side shows the screen with recognition activated. There is a textfield to set the port of the SocketServer, which must match the port set in the SpeechRemote class in the Qt application. There are two buttons for activation (Ativar/Desativar) and testing to check if recognition is working. A text box shows the decoded sentences when recognition is tested. The last component is a status bar (Label) that shows the status of the module. This class has a reference to the Speech controller that implements ConfigViewController interface, which defines the methods to update the configuration, activate and test the service.
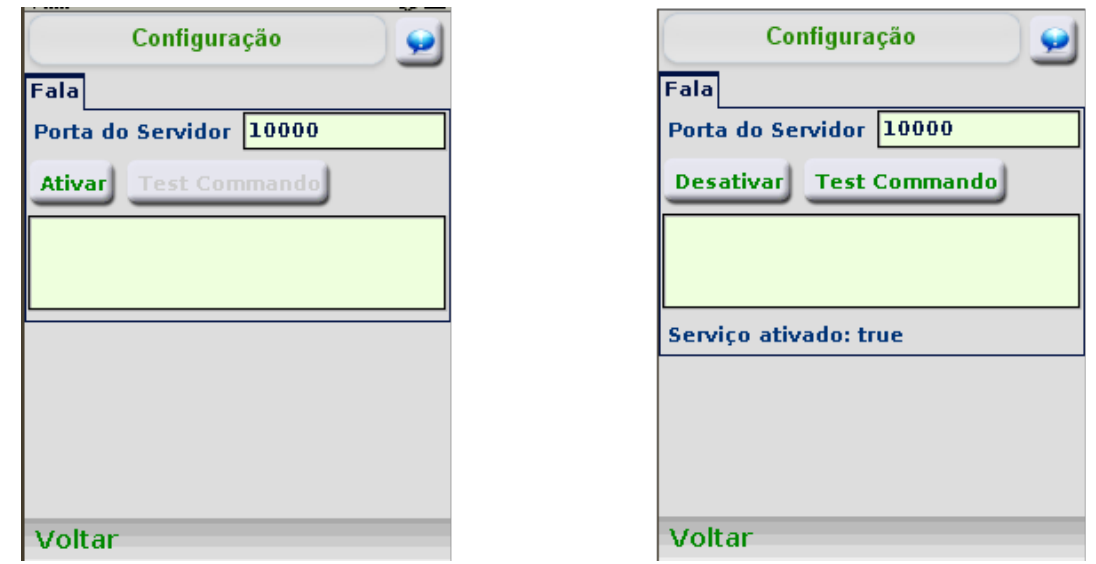

Figure C.11: Speech configuration view - Java $M E$

\section{SpeechController - Controller}

The SpeechController class coordinates the communication between the model and the views. SpeechController is a Singleton class that implements the SpeechViewController, ModelController and ConfigViewController interfaces. It has a reference to SpeechMe, SpeechDialog, SpeechConfigurationView and to a current MultimodalForm client. The IsolatedRecognition method shows the SpeechDialog to the user and requests speech recognition from SpeechMe. The decRspIsoArrived method receives the decoding status to be shown to the user. The sentenceArrived method receives the decoded sentence to be redirected to the multimodal client. Figure C.12 presents the Class diagram of this module.

Section C.3.6 presents the communication protocol to integrate the $\mathrm{C}++$ and Java applications. Section C.3.7 shows how the navigation schema in the Borboleta project was modified to support navigation using speech recognition.

\section{C.3.6 $\mathrm{C}++/$ JavaMe interaction}

A common way to integrate $\mathrm{C}++$ and Java platforms is through the Java Native Interface (JNI) [38]. JNI allows Java code to call native functions that could be in $\mathrm{C}, \mathrm{C}++$, assembler, or other languages. Java ME has a limited set of classes because of the restricted environments where it is executed. This minimized Java environment version offers a complete set of functionalities with a lower ROM footprint of less than $500 \mathrm{~Kb}$, but many key features from the original JDK, including those of JNI, are eliminated or reduced to simpler ones. It is not possible to use System.loadLibrary calls in Java ME so calling native procedures is not supported in this platform. At the Nokia Forum site, a framework to access native services 


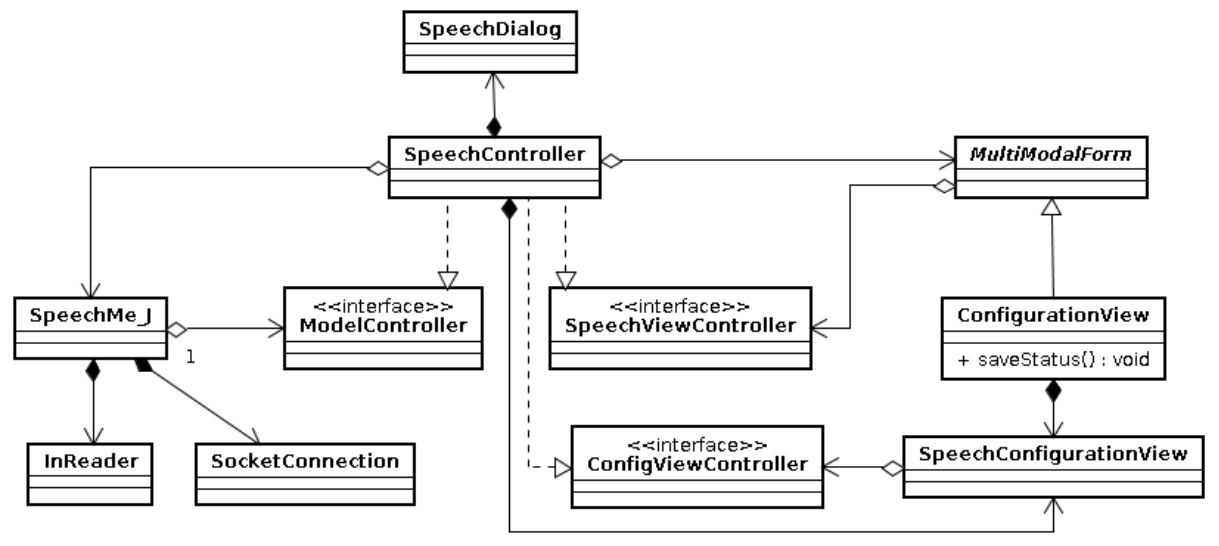

Figure C.12: Speech me - Architecture - Java ME

on mobile devices was proposed [21]. The basic idea is to create a server-client application to access native functions on the mobile platform, where the server side is developed in the native language (commonly $\mathrm{C} / \mathrm{C}++$ ) and has full access to native methods, and the client program accesses the native methods through the server using a normal communication protocol, like sockets. Based on this framework, a server-client communication schema was implemented, where the $\mathrm{C}++$ side accesses the speech decoder and provides services to other applications through socket connection in the localhost network area. Three communication processes were used over the socket connection protocol, registering a client, requesting/responding to speech recognition, and removing the client [10]. Each process has a set of defined strings to speak between both applications. The remainder of this section presents the structure of each process, its information flow and the sequence diagram. To facilitate the explanation of the interaction, the SpeechMe class will have a $\_J$ postfix that represents the Java side, or a ${ }_{-} C$ for the $\mathrm{C}++$ side.

\section{Application client registration}

To start using the speech recognition services, an application must be previously registered. To register, the socket server on the MSRS (Qt) must be running. The process starts when the user requests an activation of the service on the speech configuration view. The request is sent to the speech controller which redirects it to the SpeechMe_J class through the activateService method. SpeechMe_J opens a socket connection, and if it is successful, the InReader thread is started to process responses. At the same time, on the SocketServer, a new socket connection is open (QTcpSocket), and the SpeechRemote class handles it, instantiates and adds to its list a RemoteClient. The new client is linked to SpeechMe_C, using the Qt connection mechanism, to redirect Java recognition requests to the SpeechMe_C class during application execution in a thread-safe mode. Finally, the RemoteClient sends a rsp_cmd_reg_ok string to the Java side. The InReader receives the string and sends it to SpeechMe_J to notify the user of successful service activation. Figure C.13 shows the client registration sequence diagram.

\section{Speech recognition - request/response}

When speech recognition is activated, the speech button is enabled (Figure C.9). By clicking this button, the MultimodalForm requests the recognition service from the SpeechController. The controller redirects to SpeechMe_J, which sends a request command to the RemoteClient attaching the recognition type (isolated or continuous). The RemoteClient sends the request to SpeechMe_C, registering the client in the Msrs observers list as 


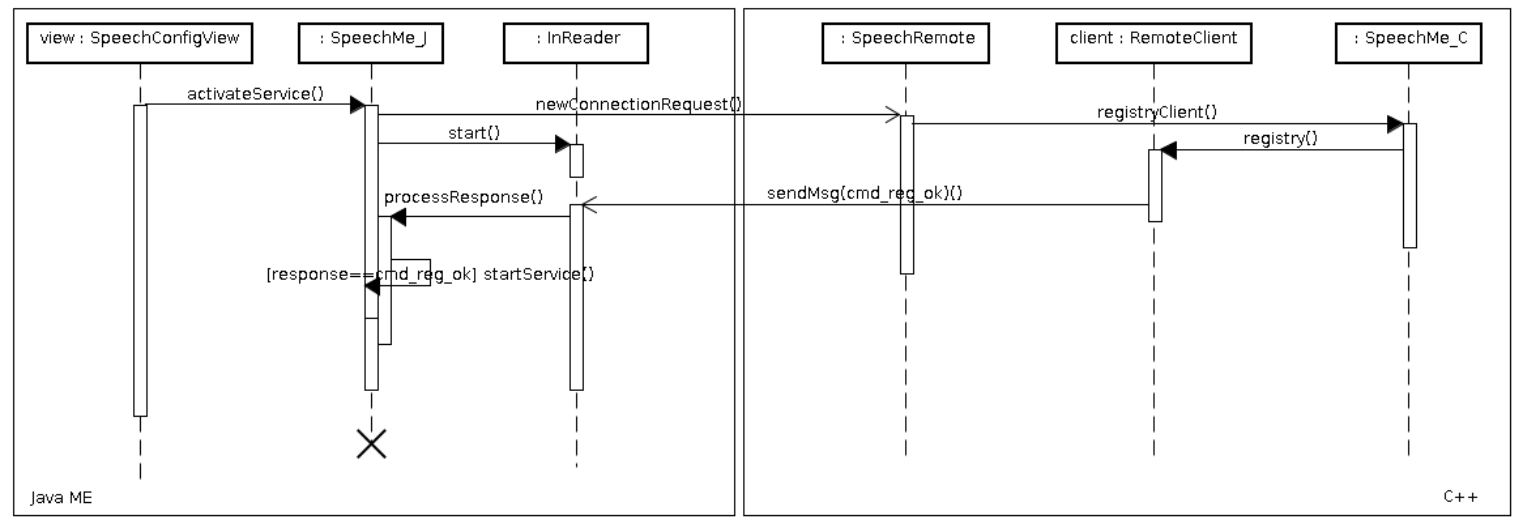

Figure C.13: Registry client

a transient observer, and emits the signal to begin live decoding in the Msrs thread. If the decoder is busy a rsp_dec_fail message is sent to the client. Otherwise, the decoding starts and constant status messages are sent to the client. The SpeechMe_J processes the messages and sends these to the SpeechController, which updates the user view. The possible status messages are ready to capture utterances (rsp_dec_ready), capturing utterance (rsp_dec_listening), decoding (rsp_dec_processing) and process finalized (rsp_dec_stopped). When the decoding is ready, the sentence is sent to the client and it is removed from the Msrs list. SpeechMe_J catches the sentence and sends it to the speech controller, which closes the speech dialog and sends the sentence to the MultimodalForm which decides how to use it. The sequence diagram of this process is shown in Figure C.14.

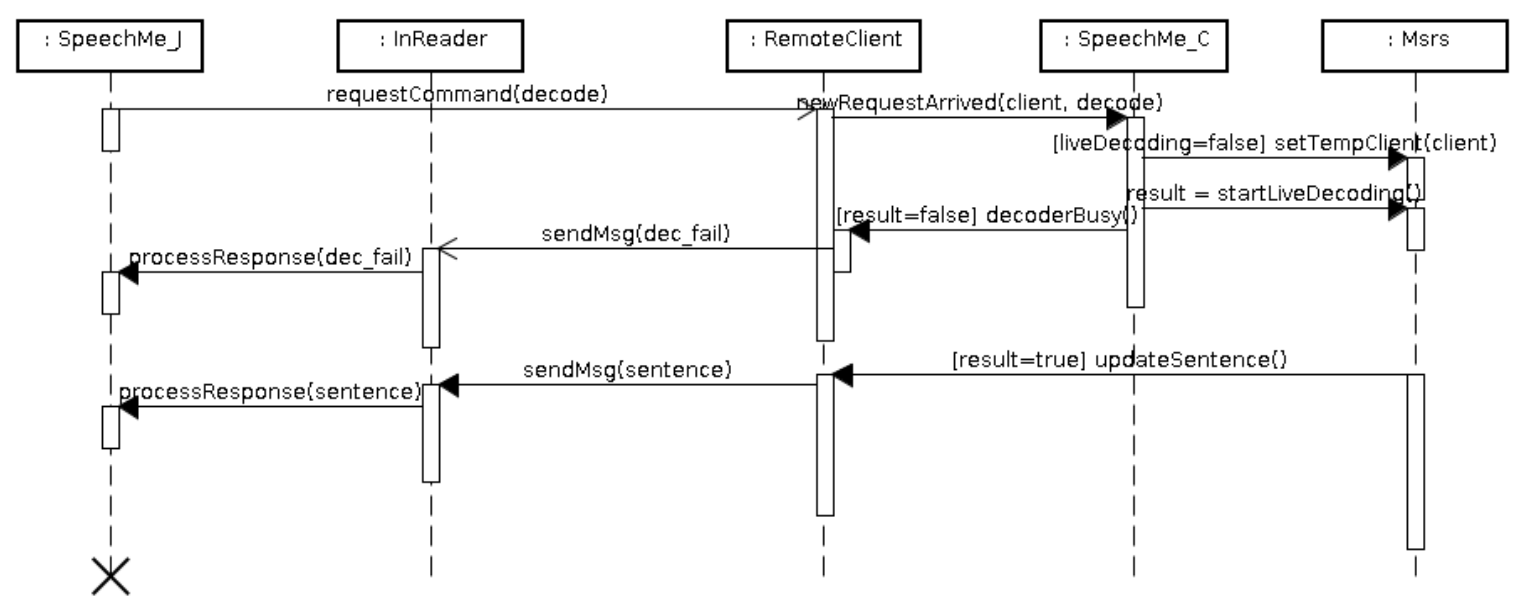

Figure C.14: Decoding process

\section{Removing the client}

When the user does not want to use the speech recognition service, it can be deactivated in the configuration form. In this case, the application must be removed from the MSRS. If the client application is closed, its reference in the MSRS must be deleted. In both cases, the procedure is the same. When SpeechMe_J receives a service deactivation request, it simply closes the socket. On the $\mathrm{C}++$ side, the RemoteClient handles this event, and requests the SpeechRemote to remove such client from its list, and it is disconnected from any signal event. Figure C.15 shows the sequence diagram of this event. When the socket is closed in the SpeechMe_J, the SpeechController disables the speech button. 


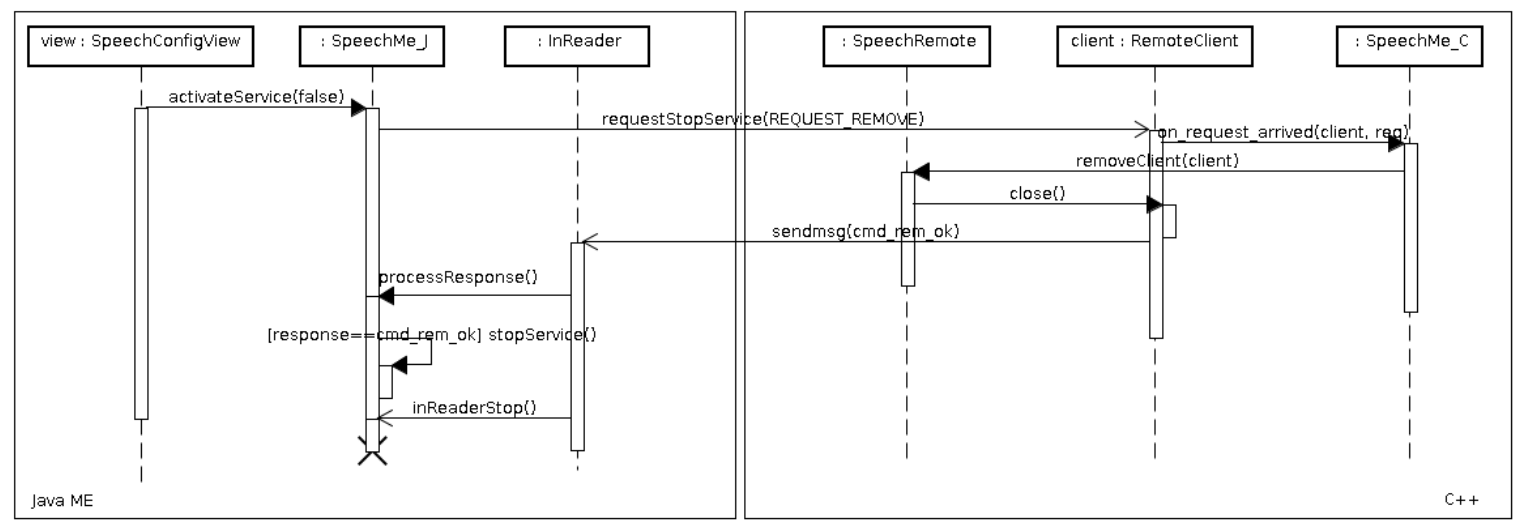

Figure C.15: Remove client

\section{C.3.7 Navigation commands structure}

Commonly, the schema to navigate through different forms on a mobile application is defined during its design, and subsequently, this schema remains static or is only slightly modified. This is the case of the Borboleta application. In the beginning, a series of functionalities and respective forms were defined. These forms were clustered and connected according to the logical steps to fulfill a task. These connections remained static. To go to a a specific form from the current window, the user used the static connections along a sequence of windows from the beginning to the end. If the user wanted to go to a form, but there was no direct link, many actions needed to be executed. Because of the small screens, not all functionalities could have a direct link. With speech recognition, the command recognition feature does not vary with the screen size, but with the available supported commands. Each form could then have direct access to any other function in the system. A series of changes were made to support random navigation using speech recogntion on Borboleta. First, a navigation redesign was carried out to improve the links between the different functionalities. Then, a methodology to support random navigation using common static navigation diagrams was developed.

During the first development phase of the Borboleta project an initial navigation diagram was defined (Figure C.16). Upon review, some weaknesses were identified: duplicated paths, non-standard names, and few shortcuts to main functions. Therefore, a new navigation diagram was designed by the Borboleta development team (Figure C.17). In the new diagram the alternative paths were eliminated, unutilized forms were deleted, standardized names were established and internationalized, and navigation facilities were incorporated, like menus and shortcuts. Having a well-defined navigation schema, the next step was to incorporate the functionality to support random navigation. The design of this functionality was inspired by a well-known and commonly used schema, dynamic Web browsing. In dynamic Web browsing, a client asks for a new page by clicking on a link in the current page. The browser sends the request to a Servlet which processes the request and redirects to the new page. In this project, the client asks for a new form by saying the desired functionality. The utterance is decoded and sent to a specific class that knows how to load the functionality. This class notifies the current form to store unsaved information and redirects to the new form. Figure C.18 shows the support for random navigation inspired by dynamic Web browsing. Since many commands are supported, two approaches were available to handle them. First, a main command controller (like a central servlet) could process all the commands and decide what new form must be loaded. Other approach was to have an individual command class handle a specific request, reducing the overloading on a simple class. In this project, the second option was selected. Sixteen command classes were created, fifteen for supported 


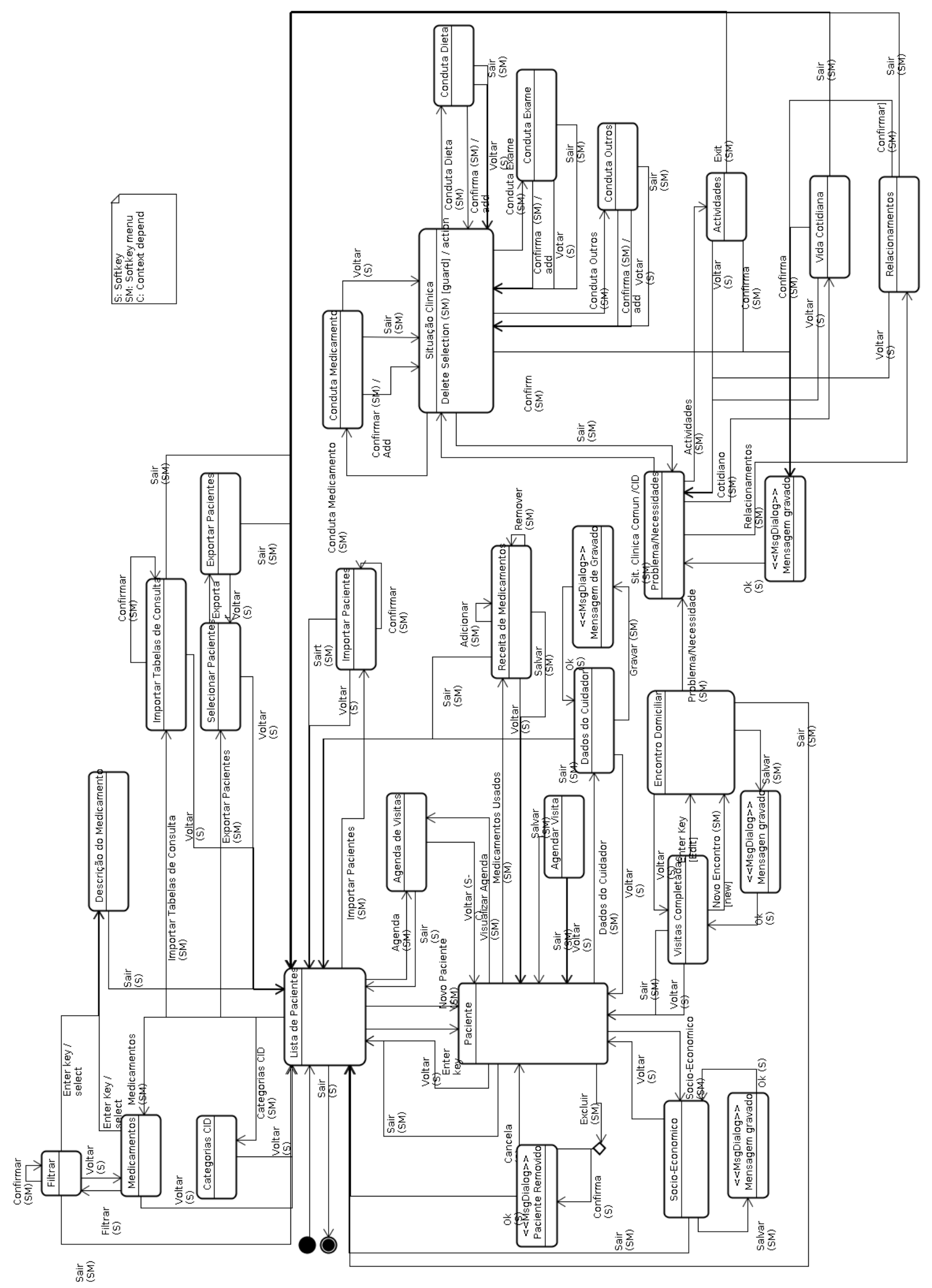

Figure C.16: Borboleta navigation diagram - Initial 


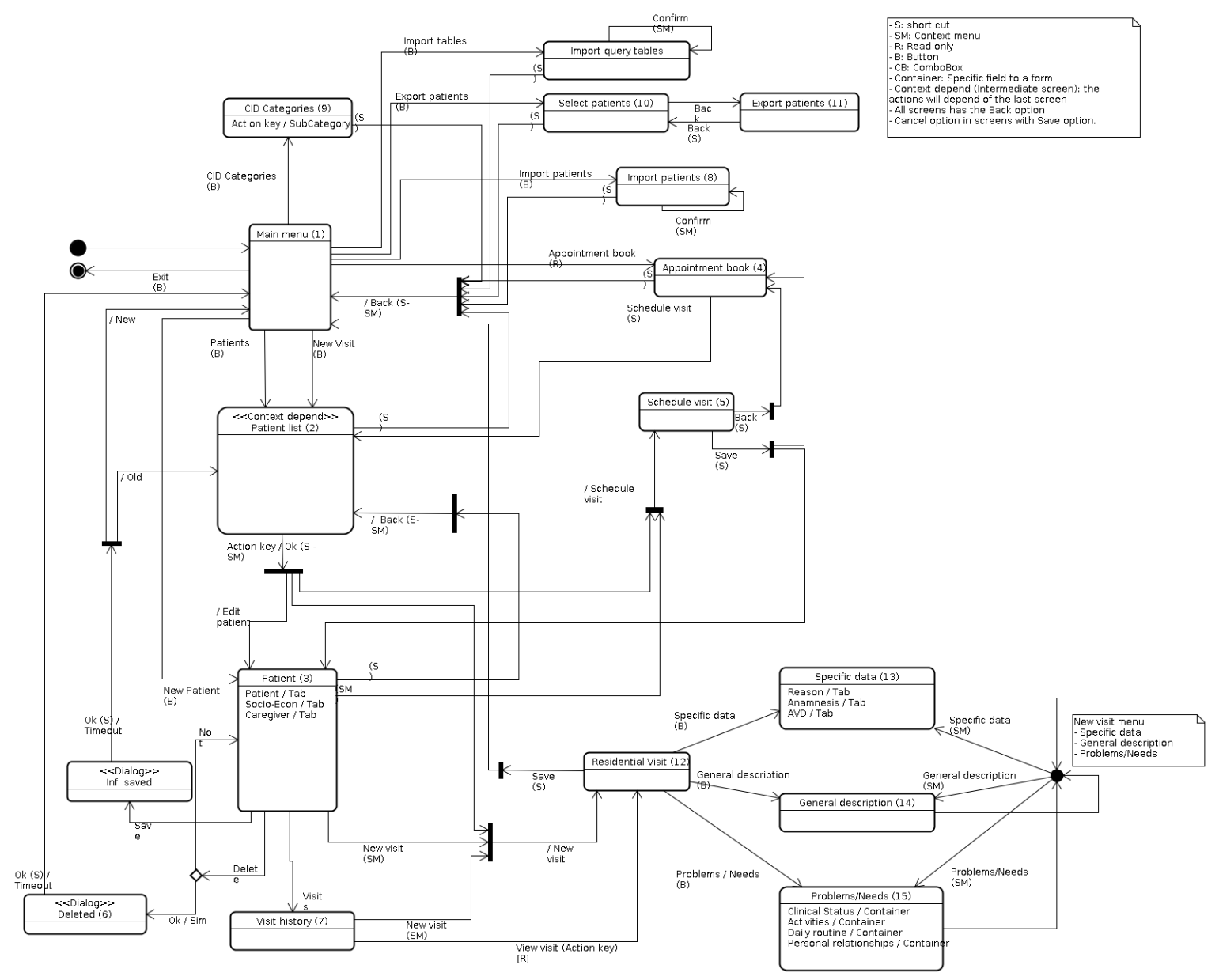

Figure C.17: Borboleta navigation diagram - Final 
commands and a null command to catch unknown commands (Null Object pattern [22]). In order to instantiate a correct command for a given utterance, a basic word processor was implemented. The SpeechCommands class processes a sentence by checking if it matches a model in the dictionary. If so, a MMCommand is instantiated using a single class factory [22].

Dynamic Web browsing

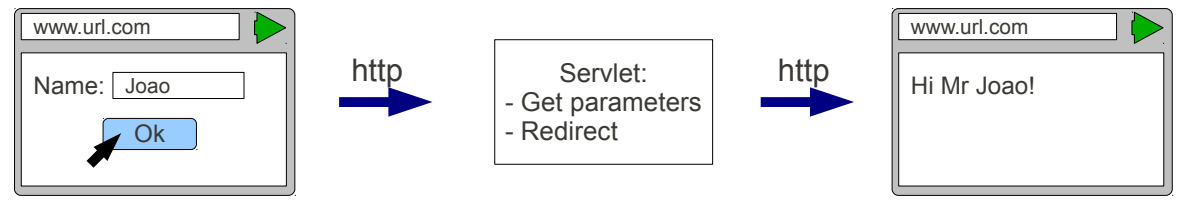

Borboleta random navigation
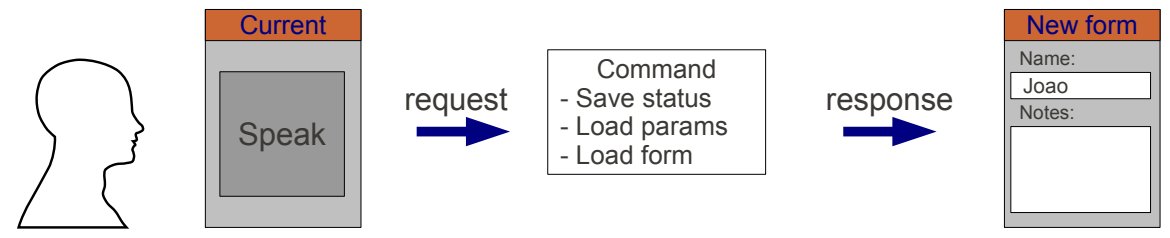

Figure C.18: Random navigation

Another characteristic inspired by Web browsing was the use of contexts to get required parameters for the next form. In dynamic Web browsing using a Java paradigm, a Servlet or a JSP page can save or access information in a specific context, such as request, page, or session context. In a given context, a Servlet could know which data is available, which must be retrieved or which must be asked for from the client. For Borboleta, three contexts were defined (Figure C.19): 1) a general context that includes all the forms, 2) a patient context that is available when a patient is selected, and 3) a visit context available when visits are being done. If a command is executed in a given context, it will know which information is available or if it needs to show an intermediate form to gather required information to complete the command. For example, if a New visit command is executed in the general context, it will show the Select a patient screen to find out who the patient is for the medical visit. If the same command is executed in the patient context, the new visit form is loaded immediately, because the patient variable is known in this context. Some commands are only available in specific contexts. For example, Problem $\&$ needs, Specific data, General description and Final program are used only in the context of medical visits. If any of these commands is captured out of the medical visit context, they are ignored. When the sentence is decoded and sent to the SpeechController, it redirects the string to the MultimodalForm, where the word processing is carried out by the SpeechCommands class, and a command is instantiated to execute the tasks to load the required functionality. Figure C.20 presents the class diagram for the commands structure. 


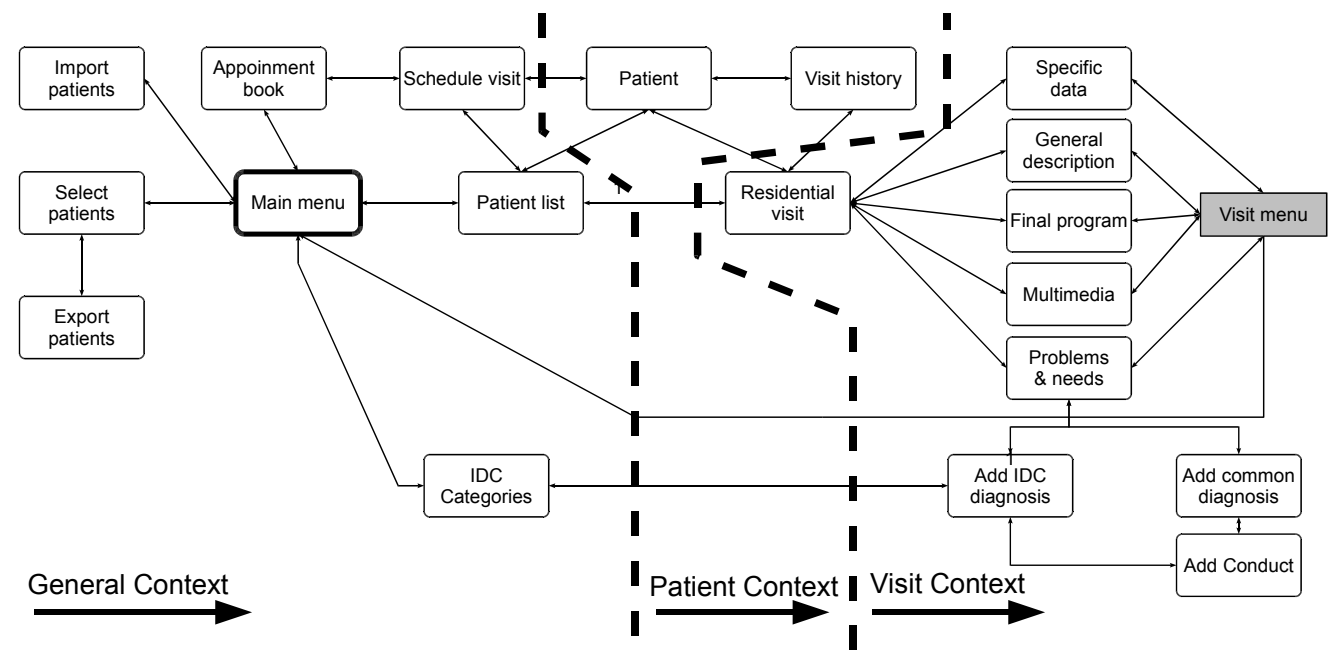

Figure C.19: Navigation diagram contexts

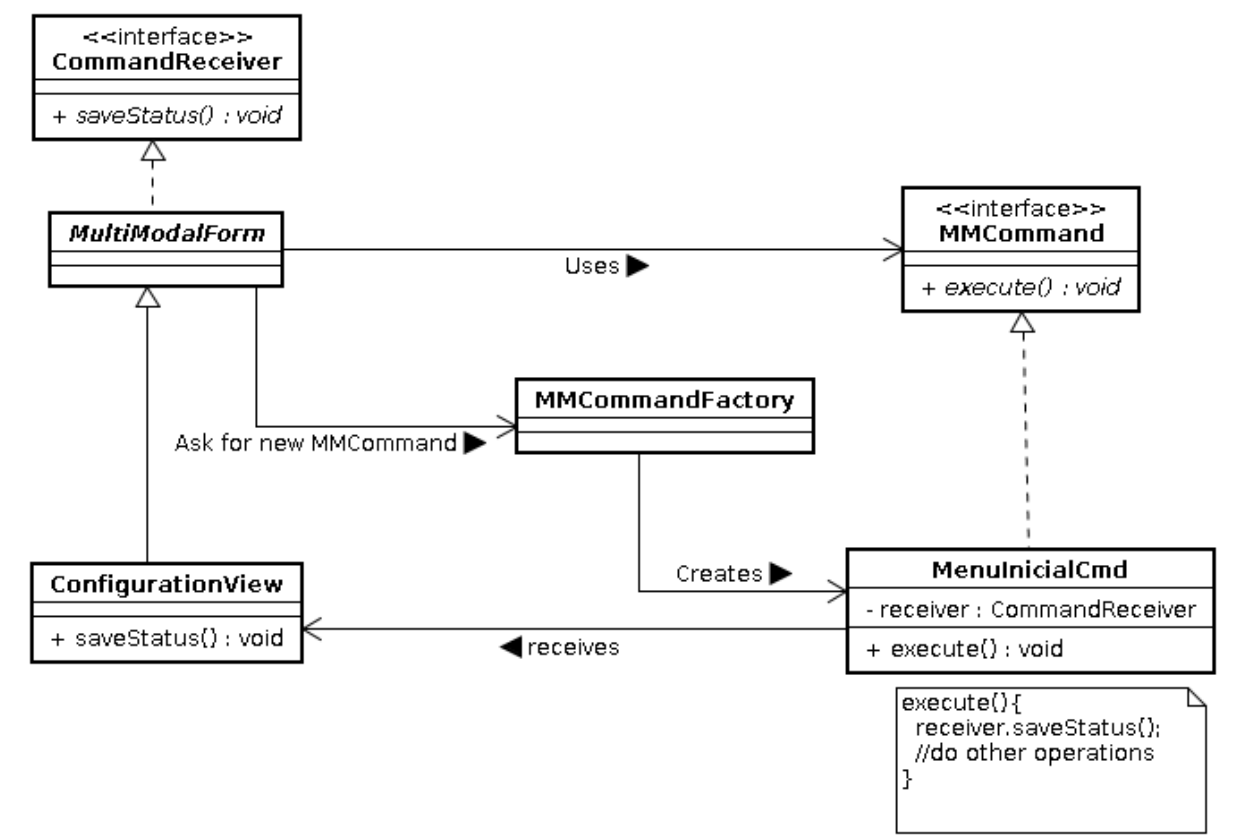

Figure C.20: Commands Structure 


\section{Appendix D}

\section{Evaluation}

System validation involved assessing three relevant aspects: speech recognition accuracy, system performance and usability. System performance and accuracy is related to the system's ability to identify utterances correctly with an acceptable time range and optimal resource usage. Usability evaluates how software functionalities are accessed by the users, the complexity of the system and user acceptance. This chapter presents the evaluation methodology for the different aspects and the collected results, including how the evaluation was done, the parameters taken into account and the metrics used. The experiment design was based on a systematic approach for performance evaluation proposed by Jain [32]. Jain proposes a ten-step methodology to evaluate system performance, covering: definition of goals, outcome values, system parameters and metrics, experiment design, and analysis and interpretation of results. The proposed methodology was adapted to our requirements of speech recognition and system performance evaluation, so some steps were omitted or modified.

\section{D.1 Speech recognition accuracy}

Typically, an automatic speech recognition system (ASR) is evaluated using the word error rate. WER measures the system accuracy by contrasting recognized utterances with real transcriptions. WER was used to compare isolated word, phone-based and whole-word modeling approaches. The approach with the lowest WER was selected to be included in the system. Two types of experiments were performed to evaluate the accuracy. The first was batch testing, where audio files from the collected data were used as utterances to be processed by Pocketsphinx. The decoded sentence is used to calculate the WER. The second was a live decoding experiment, where models were tested using live utterances from the health care team. Below, the complete description of the experiments for batch testing as well as live decoding testing is presented. In both experiment types the accuracy metrics were the WER and the sentence error rate (SER), this last value is calculated by Pocketsphinx and it is similar to the first one, but it is related to the full word string rather than to individual words. Commonly, speech recognition accuracy is evaluated just with WER, but since this work focuses on navigation commands, which could be a single word or a string of words, the SER is a better metric to compare results across the four approaches.

\section{D.1.1 Batch testing}

Seventy percent of the collected data was used to train the acoustic models, the remaining $30 \%$ was used to test them. According to the four approaches for creating acoustic models, defined in training section (See Section C.2.2), four experimental approaches were created to find the one with the lowest error rate. The testing was done in two places: 1) a desktop 
computer where acoustic models were trained and 2) on the Symbian platform emulator to validate desktop WERs. Pocketsphinx provides a batch decoding tool to get WER values automatically after training (psdecode.pl, pocketsphinx_batch). For the Symbian platform, batch decoding was done on the emulator, but because Symbian does not support Perl scripts, decoding results were processed on the desktop machine to calculate the error rates.

For the experiments, the system was defined as the Pocketsphinx decoder running on the desktop and on the Symbian emulator, and the goal was to find the acoustic and language model set that minimizes the WER. The system parameters are the test data (165 utterances), the four approach configurations for recognition, and the acoustic models generated in the training phase. The quantity of acoustic models depends on the combination of the number of states per HMM, and whether it skips states or not. From the parameters, two factors were selected for evaluation, the number of states in the HMM (3 or 5), and the skipping states flag (true or false). Multiplying the four approaches by the choice of two states per HMM and by the two options in the skipping states results in sixteen experiments to find the lowest error rate. Table D.1 presents the experiment list. Once the lowest WER acoustic model was selected, it was tested on the Symbian emulator to verify the accuracy in the Symbian environment.

Table D.1: Accuracy experiments - Batch testing

\begin{tabular}{|c|c|c|c|c|}
\hline \multirow{2}{*}{ States/Skip } & Approach 1 & Approach 2 & Approach 3 & Approach 4 \\
\cline { 2 - 5 } & $\begin{array}{c}\text { word-based AM } \\
\text { word-based LM }\end{array}$ & $\begin{array}{c}\text { phoneme-based AM } \\
\text { word-based LM }\end{array}$ & $\begin{array}{c}\text { word-based AM } \\
\text { command-based LM }\end{array}$ & $\begin{array}{c}\text { phoneme-based AM } \\
\text { command-based LM }\end{array}$ \\
\hline $3 /$ no & exp. 1 & exp. 2 & exp. 3 & exp. 4 \\
\hline $5 /$ no & exp. 5 & exp. 6 & exp. 7 & exp. 8 \\
\hline $3 /$ yes & exp. 9 & exp. 10 & exp. 11 & exp. 1 \\
\hline $5 /$ yes & exp. 13 & exp. 14 & exp. 15 & exp. 16 \\
\hline
\end{tabular}

AM: Acoustic model - LM: Language model

\section{D.1.2 Live decoding testing}

Final evaluation of recognition accuracy was executed with real users using the range of mobile devices used in the Borboleta project. Prior to this testing, a live decoding test was carried out in the laboratory to verify if the models with the best accuracy had the same behavior on mobile devices. This test was executed by performing live decoding with native speakers of Brazilian portuguese, using the continuous mode in SpeechMe and reading commands. These talkers did not belong to the group that recorded the samples for training. The five models with the lowest WER and SER from batch decoding were selected for live decoding. Five people (3 male, 2 female) were used to test live decoding. The acoustic and language models from the experiment with the new lowest average error rate was used to carry out the accuracy and usability tests with healthcare professionals. This evaluation is described in Section D.3.

\section{D.1.3 Accuracy results}

Table D.2 presents the SERs and WERs for the sixteen experiments for batch decoding testing. Experiment 6 presented the lowest WER (4.5\%) and SER (4.2\%). The 5 experiments with the lowest error rates (in bold face on table) were used to carry out the live decoding testing in the laboratory. Table D.3 presents the results for live decoding testing with people in laboratory. Experiment 6 continued to have the lowest WER (3.6\%) and SER (4\%), thus, acoustic and language models from this experiment were used in the accuracy testing during 
the usability evaluation. Live decoding testing in laboratory was carried out with models from

Table D.2: Accuracy results - Batch decoding

\begin{tabular}{|c|c|c|c|c|}
\hline States/Skipping & Approach 1 & Approach 2 & Approach 3 & Approach 4 \\
\hline & SER / WER & SER / WER & SER / WER & SER / WER \\
\hline 3 / no & ${ }^{1} 22.5 / 17.0$ & ${ }^{2} 10 / 10$ & ${ }^{3} 17.5 / 14.5$ & ${ }^{4} 19.2 / 20.5$ \\
\hline $5 /$ no & ${ }^{5} \mathbf{9 . 2} / \mathbf{6 . 0}$ & ${ }^{6} \mathbf{4 . 2} / \mathbf{4 . 5}$ & ${ }^{7} 8.3 / 8.5$ & ${ }^{8} 9.2 / 11.0$ \\
\hline 3 / yes & ${ }^{9} 21.7 / 14.0$ & ${ }^{10} 25.8 / 26$ & ${ }^{11} 17.5 / 13.0$ & ${ }^{11} 35.8 / 39$ \\
\hline 5 / yes & ${ }^{13} \mathbf{8 . 3} / \mathbf{6 . 0}$ & ${ }^{14} \mathbf{5 . 8} / \mathbf{5 . 0}$ & ${ }^{15} \mathbf{8 . 3} / \mathbf{6 . 0}$ & ${ }^{16} 14.2 / 15.6$ \\
\hline
\end{tabular}

experiments 5, 6, 13, 14 and 15. Experiments 5 and 13 belong to approach 1, experiments 6 and 13 belong to approach 2. These two approaches have dictionaries with 24 words because commands were compose of individual words (e.g., CARACTERIZACAO GERAL is a command with 2 words). Approach 3 (experiment 15) has a dictionary with 15 words because commands were represented as a single word (e.g., CARACTERIZACAO_GERAL is a single word/command). Thus, WER for experiments 5, 6, 13 and 14 was calculated with 24 words as reference, and WER of experiment 15 was calculated with 15 words as reference. Since using WER to compare all the experiment is not possible because the first four were based on 24 words and the last on 15 words, analysis was based mainly in the SER that is base on 15 commands for all experiments.

Table D.3: Accuracy results - Live decoding in Lab.

\begin{tabular}{|c|c|c|}
\hline Experiment & SER(\%) & WER(\%) \\
\hline 5 & 17.3 & 22.2 \\
\hline 6 & 4.0 & 3.6 \\
\hline 13 & 18.7 & 23 \\
\hline 14 & 10.1 & 8.3 \\
\hline 15 & 20 & 23.3 \\
\hline
\end{tabular}

\section{D.1.4 Analysis of results}

One of the objectives of the present project was to evaluate which of the proposed approaches for speech recognition presented the high accuracy results in command recognition. Based on the results from batch and live decoding testing (Tables D.2 and D.3), several aspects were relevant to accomplish such objective. Doing a horizontal analysis along the four approaches, the WER/SER sets were lower for approaches 1 and 2 in most of the experiment than approaches 3 and 4. Approaches 1 and 2 used dictionaries with separated words and a language model where commands are composed of these separated words, this configuration presented better results than approaches where joining two or more words represented a single command, independently whether the words were decomposed in phonemes or not. Contrasting approaches with words decomposition in phonemes or whole words, accuracy variations depend of the specific approach. Going from whole word representation to phoneme representation in approaches 1 and 2, the WER and SER were reduced significantly, with exception of having three states with skipping set to true (experiment 10), where error rates increased. In approaches 3 and 4, going from whole word to phoneme representation, the WER and SER was higher in all cases. Taking into account the later two findings, it could be said that for command recognition it is better to have phoneme word representation for acoustic models and language models of separated words for FSG models.

Having five states in the acoustic model instead of three states reduces the error rate for all approaches regardless of whether skipping states is allowed or not. Focusing on the skip 
states parameter, accuracy worsened or remained the same when skipping states was allowed. In general, using five states and no skipping offers better results.

Based on the results obtained in these section, for speech recognition of commands, using separated words for modeling FSG languages and phoneme word representations with five states and not allowing skipping states, acoustic modeling presents the best results. For this project, experiment 6 had these characteristics and presented the lowest error rates, thus, it was used in the system. Results in table D.3 show that results from batch decoding testing are valid for live decoding, but WER increased due to the additional noise from laboratory and PC speakers. Nevertheless, with real people, WER and SER had a small improvement, from $4.5 \%$ to $3.6 \%$ and from $4.2 \%$ to $4 \%$ respectively.

\section{D.2 Software performance}

Recognition performance includes recognition speed and resource usage, an important aspect in mobile environments with few processing and storage resources, and battery-life dependency. The results from the performance tests helped us to decide whether it was feasible to have a speech recognition system incorporated with Borboleta on the mobile device. In order to further characterize software performance, four aspects were evaluated: CPU and memory usage, response time and battery life estimation. To collect the performance data on target devices, the Nokia Energy Profiler software was used (Figure D.1). This profiler is a stand-alone application that monitors resource usage in real time on Symbian devices. With this software it is possible to monitor power consumption, battery voltage and current, CPU and RAM usage, network speed, mobile-network signal strengths, 3G timers, and WLAN signal strength.

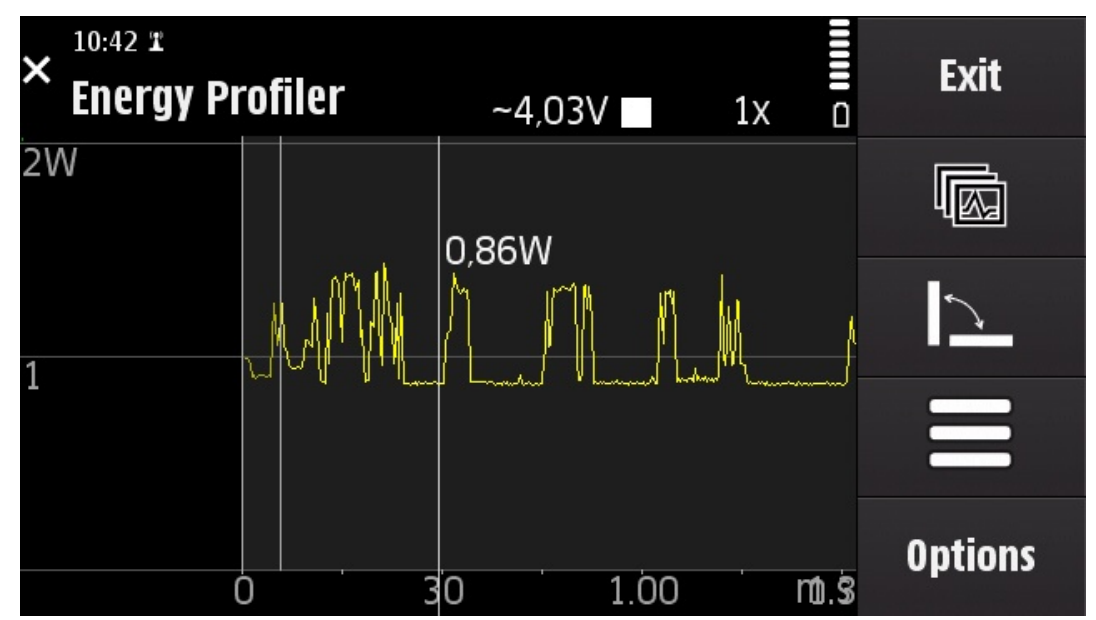

Figure D.1: Nokia Energy Profiler

For processor activity and memory consumption, three places were defined along the SpeechMe execution path. The first point was language initialization when loading acoustic and language models and initializing the decoder. The second was the waiting/decoding phase, when the system is ready to provide speech recognition services. The third was the stopping phase, where resources are released. For response time, the analysis was focused on the decoding task. To perform accurate speech recognition the microphone must be calibrated which takes at least 3 seconds. For this project, the calibration phase was done during the first decoding request and omitted after that because of the time delay. The first decoding is not as accurate because the device is not calibrated, but for subsequent decodings the error rate is as presented in the accuracy results (see Tables D.2, D.3 and D.8). The response time was evaluated as the time used to process and decode the collected utterance. During the full 
SpeechMe execution the estimated battery life time was monitored to evaluate the decoder impact.

The experiment was carried out on the Symbian device as follows. After the Borboleta software was started, the Nokia Energy Profiler was started and the monitoring initialized. Then, a specific task was executed every 30 seconds in order to identify the changes in the collected data during the analysis. The following are the tasks executed in sequence to collect the data:

1. Launch SpeechMe.

2. Initialize the decoder (Load acoustic and language models).

3. Start an isolated recognition task.

4. Start speech server (Socket server).

5. Connect to SpeechMe from Borboleta.

6. Request speech recognition from Borboleta.

7. Close connection from Borboleta and close SpeechMe (Release resources).

Once the task was finished, the profiler is stopped. Results are exported to CSV format for further analysis.

\section{D.2.1 Performance results}

Figure D.2 presents percentage of memory used during the experiment based on a total of $128 \mathrm{MB}$. Figure D.3 presents the CPU consumption. 8Mb for RAM were assigned for Speech Me at the beginning of the application. This quantity guarantees good performance on devices with low capabilities (e.g., Nokia N95) which perform speech recognition without the SpeechWeb functionality. On new generation phones (e.g., Nokia N97 or N8), all functionalities of SpeechMe are available. CPU usage is larger during model initialization, which is done once during the execution of the application, and during recognition and execution of commands on the Borboleta side. Figure D.4 merges memory and CPU usage. Intervals of time correspond to the defined tasks above.

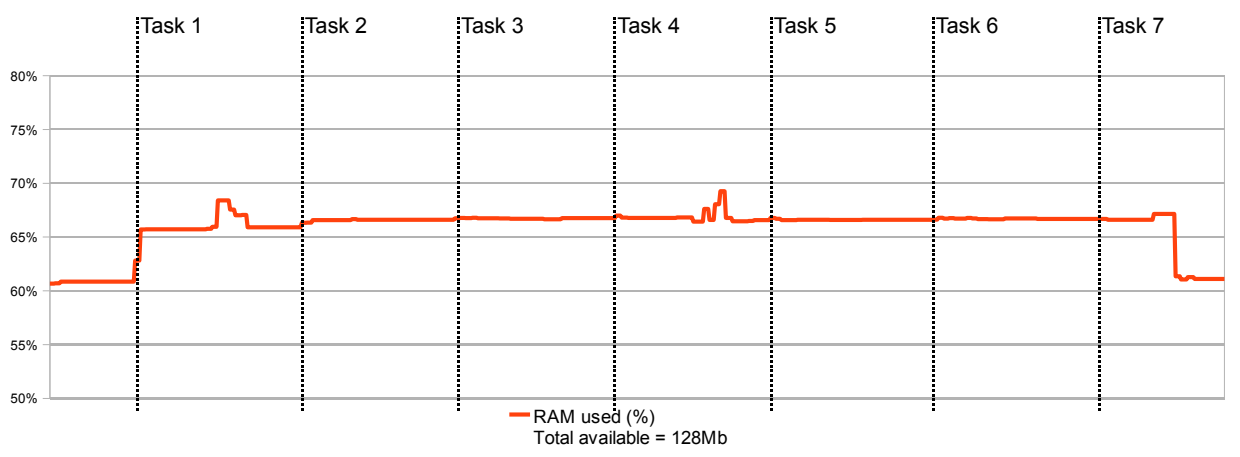

Figure D.2: Memory usage for SpeechMe and Borboleta

Table D.4 presents the response time for language initialization and speech recognition on a Nokia N95 and on a Nokia N97. The decoding was performed directly on SpeechMe (SpeechPad) and also by requesting the service from Borboleta. The decoding time was measured in two ways. First, the total time is measured including requesting the service, speaking the command and getting the response. Second, the response time is measured from the processing state (utterance has been collected) until the utterance has been decoded and the command has been executed. 


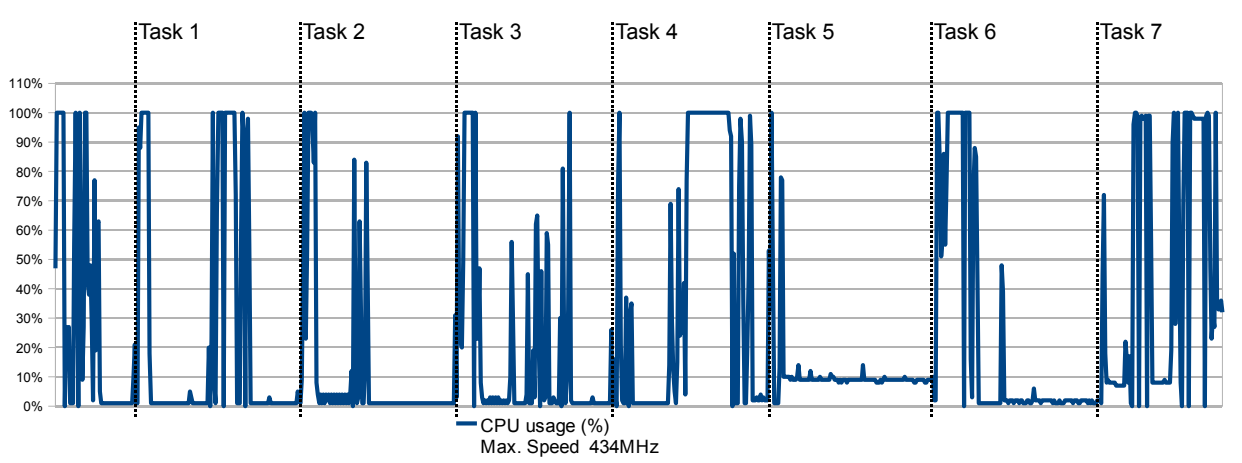

Figure D.3: $C P U$ usage for Speech Me and Borboleta

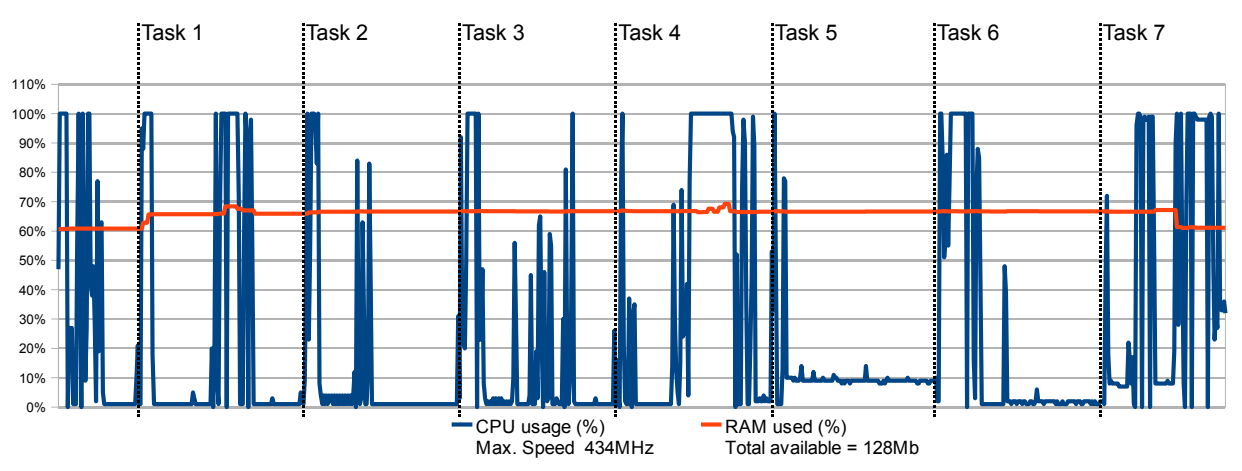

Figure D.4: $C P U$ and memory usage merged

Table D.4: Response time - decoding performance

\begin{tabular}{|c|c|c|c|c|}
\hline Task & \multicolumn{2}{|c|}{ N95 } & \multicolumn{2}{c|}{ N97 } \\
\hline Task 3: Language initialization - resp. time & \multicolumn{2}{|c|}{6250} & \multicolumn{2}{c|}{2834} \\
\hline & full & dec+exe & full & dec+exe \\
\hline Isolated decoding (SpeechPad) & 5660 & 1220 & 3025 & 814 \\
\hline Decoding a command (From Borboleta) & $\approx 30(\mathrm{sec})$ & 6120 & 5032 & 1228 \\
\hline
\end{tabular}

dec+exe: Decoding plus command execution

Time in milliseconds 


\section{D.2.2 Analysis of results}

As shown in Figure D.2, SpeechMe reserves $5 \%$ (6.4Mb) of available memory during initialization. During file browsing using the Qt file selector, which has it own memory space, a maximum of $10.24 \mathrm{Mb}$ are used $(9 \%)$, then memory usage average is $6 \%(7.6 \mathrm{Mb})$. The peak between tasks 4 and 5 was attributed to a Form instantiation on the Java side. At the end of the execution all resources are released. Additional resources could be required for other applications to interact with SpeechMe, as when Borboleta instantiates forms when executing speech commands. As shown in Figure D.3, SpeechMe CPU usage in general was very light. Initialization (Task 1) was fast and did not use much CPU time. Between Tasks 1 and $2 \mathrm{CPU}$ peaks were found due to Qt file browser initialization, which is used to select the configuration file. CPU time in Task 3 corresponds to configuration loading and decoder initialization, it has a low CPU usage as confirmed in Table D.4 (2834 ms). Peaks between Tasks 2 and 3 were generated for widget changing from Config to SpeechPad. The isolated recognition (Task 3) uses the CPU twice, first while opening the microphone to start capturing and a second, larger time when processing and decoding. Between Tasks 3 and 4 peaks were generated due to widget changing from SpeechPad to Config in order to start the socket server. Task 4 corresponds to server initialization and the procedure uses little CPU resources. The large CPU usage interval between Tasks 4 and 5 is caused by going from SpeechMe to Borboleta and opening the Configuration form on the Java side. The socket connection (Task 5) is fast, and most of the time is used to request network permissions from the user. The command recognition procedure (Task 6) covers the SpeechDialog presentation to the user, requesting speech recognition service, updating the state in the dialog, getting the results, and executing the command to change to a new Form. Closing connections and SpeechMe finalization used acceptable CPU resources (Task 7). During the experiment the battery voltage level was monitored as shown in Figure D.5. The voltage level presented a small reduction from $4.02 \mathrm{~V}$ to $3.98 \mathrm{~V}$, taking into account that such reduction includes the decoder initialization and the speech recognition. In normal usage, the decoder initialization is supposed to be initialized once. In general, the system performance is within the limits of normal applications. There are not active loops and no extra processing is done during SpeechMe execution.

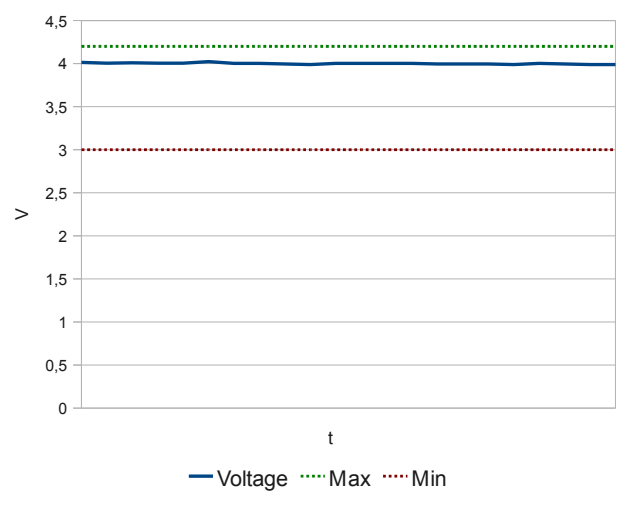

Figure D.5: Battery voltage levels

\section{D.3 System usability - Borboleta case study}

For usability evaluation, a typical usability testing approach was used based on Sharp et al. The approach aims to insure consistency in navigation schemes, system responses, and system vocabulary [56]. A preliminary navigation evaluation was performed before 
integration of ASR with the system. The same navigation evaluation using speech recognition was also executed. Both results were compared to get usability results and to evaluate the usefulness and usability of including ASR in Borboleta. In the usability evaluation for speech recognition a set of tasks were executed by the user. After each task, the users completed an after scenario questionnaire (ASQ). At the end of the test, each users completed a poststudy system usability questionnaire (PSSUQ) [56]. Questions included statements like "The system was easy to use" and "I successfully completed my task" evaluated on a 5-point Likert scale [39].

This section describes the proposed methodology to test the usability in the navigation schema for the Borboleta system in order to implement speech recognition capabilities. The testing covers two phases, an initial usability test that evaluates the navigation system without speech recognition (non-multimodal), and a post-installation test to validate the effectiveness of the speech recognition features (multimodal).

\section{D.3.1 Experiment design}

The objectives of the usability testing are to:

- evaluate the navigation through the Borboleta system to get initial usability results without speech recognition features, and

- test the system navigation schema using speech recognition to validate if this feature improves the user experience in the system usage.

\section{Test description}

The target public are the users of the system in the health care center. Each user performed up to five trials of reaching predefined target screens from different starting points in the system by using any of the interaction elements in the application. During this task, the user can receive help about how to reach such screen, but any help was recorded by the researcher. In each trial, the following information was collected:

- time to complete the task,

- path followed,

- quantity of clicks during the task, and

- any user comments or suggestions while performing tasks.

At the end of the experiment, the users evaluated their experience using the system during the navigation on a 1-5 Likert's scale where lower values mean greater acceptance level. A mobile testing project using a similar technique can be found in [56]. The questionnaries are shown in Appendix J.

As shown in Figure D.6, the start-target path is defined as a series of steps that the user must follow (continue black line), but in some cases, there are alternative paths to reach the same target screen (dashed line). Alternatives paths are present because of the shortcuts added to the system to reduce the complexity in the navigation or because a given screen can have different internal functions (multi-functional). For example, the Patient screen shows the data of saved patients as well as shows the form for new patients.

\section{Task definition}

The task definition is based on the navigation schema shown in Figure D.7. A task is a duple of a starting screen and a target screen in a specific scenario. In the case of 


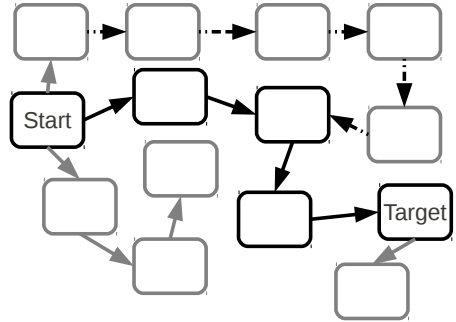

Figure D.6: Path to reach the screen target

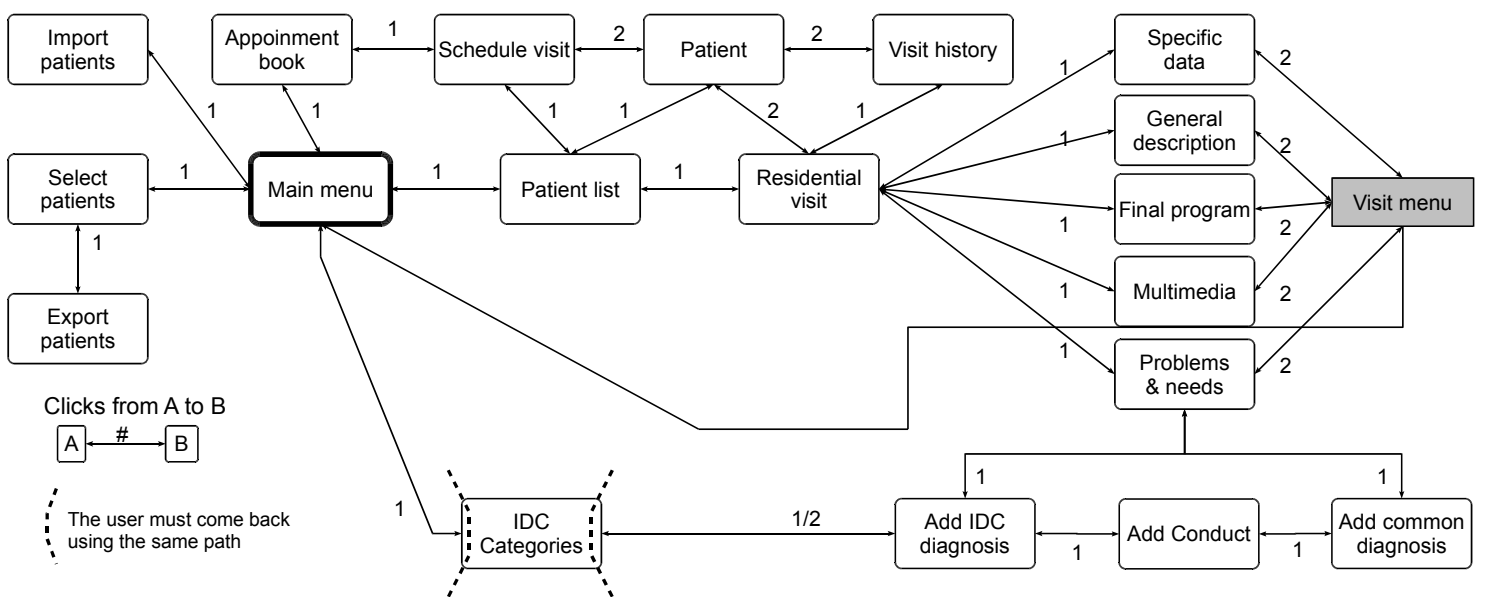

Figure D.7: Navigation schema - Borboleta project

multi-functional screens, that is screens that execute different procedures depending on the initial source, there was an additional parameter indicating the required function. Five tasks were defined covering most of the application screens and are described below. Table D.5 summarizes the selected tasks, indicating the initial screen, target screen, a specific function in the case of multi-functional screens, and the path length, that is, the number of user actions to move through the screens to reach the target based on the navigation schema and on-screen actions like Patient selection.

Task 1: Scheduling visit The health care professional is collecting data during the residential visit and is currently in the Specific-data section. At this moment, the patient talks about a future engagement, so the professional decides to go to the Appointment-book and schedule the next visit in order to avoid time conflicts. The initial screen is Specific-data, the target is Schedule-visit without optional functions.

Task 2: Checking visit history list During the execution of a residential visit, the health care professional would like to see the visit history list in order to view an older record or just to confirm if a visit was done on a specific date. The professional is in the General-description screen and wants to go to the Visit history screen to check previous visits.

Task 3: Seeing patient's information The health care professional is checking the patient and wants to add a new common diagnosis. Before adding the diagnosis, it is important to review some of the patient's medical information, accessible from the Patient screen. The professional has to navigate from Add-common-diagnosis screen to Patient screen.

Task 4: Viewing an old visit entry In order to add an IDC diagnosis the health care professional wants to verify a previous residential visit to get patient history and check 
Table D.5: Task list for usability testing

\begin{tabular}{|c|c|c|c|c|}
\hline Task & Starting screen & Target screen & Function (Optional) & Length \\
\hline 1 & Specific data & Schedule visit & - & 5 \\
\hline 2 & General description & Visit history & - & 6 \\
\hline 3 & Add common diagnosis & Patient & - & 5 \\
\hline 4 & Add IDC diagnosis & Residential visit & View old visit & 8 \\
\hline 5 & Appointment book & New visit & New visit & 3 \\
\hline
\end{tabular}

patient's evolution. To reach the target, the professional must move through the system from the Add-IDC-diagnosis to Residential-visit, but in this case the target screen will show saved data selected from Visit-history.

Task 5: Creating a new visit In the beginning of the residential visit, the professional is checking the Appointment-book, then will start the new visit by going to the Residential-visit screen, but in this case an empty form for the new visit is shown.

\section{Usability questions}

The usability questions were based on the mobile phone usability questionnaire (MPUQ) involving 6 factors: learnability and ease of use, problem solving capabilities, multimedia properties, minimal memory load, control and efficiency and typical tasks for mobile phones [52]. The questions were divided in two groups for our experiments, a group that asks for specific information for each task, and a general question group that asks about the system and its performance during the test. There are three questions asked about each of the five tasks. For the general group, five questions were created, resulting in a questionnaire composed of fifteen task questions, five general questions and two open-ended questions to ask the user for comments and suggestions. Below are the questions for each group. Appendix J presents the full questionnaire in English and Portuguese

\section{Task-specific questions}

1. Did you complete the task?

2. What was the difficulty level to complete the task?

3. Number of clicks

\section{General questions}

1. How was the navigation through the system?

2. What do you think about the structure of the application?

3. Did you find the features you wanted in the place you expected?

4. Is it easy to navigate between hierarchical menus, pages, and screen?

5. Do you feel comfortable and confident using this product?

In the post-installation test, question 4 was changed to "Was the navigation using speech recognition easy to use?" 


\section{D.3.2 Usability results}

Usability tests were carried out with four people ( 1 male, 3 female) from the healthcare team. Tables D.6 and D.7 present the information collected during the initial usability testing (non-multimodal). The first one is for task-specific questions, and the second are the results for general questions. Tables D.8 and D.9 present the results for the post-installation testing (multimodal). The error rates for the acoustic model used (experiment 6) were 5\% SER and $4.2 \%$ WER.

Table D.6: Usability testing results - Specific questions - Non-multimodal

\begin{tabular}{|c|c|c|c|c|c|c|c|c|c|}
\hline \multirow[b]{3}{*}{ Tasks } & \multicolumn{8}{|c|}{ Questions } & \multirow[b]{3}{*}{ Time $^{\S}$} \\
\hline & \multicolumn{2}{|c|}{$1^{*}$} & \multicolumn{5}{|c|}{$2^{*}$} & \multirow{2}{*}{$\begin{array}{c}3^{+} \\
\text {Clicks }\end{array}$} & \\
\hline & Yes & No & 1 & 2 & 3 & 4 & 5 & & \\
\hline 1 & 100 & & & 25 & 75 & & & $\overline{77}$ & 8.3 \\
\hline 2 & 100 & & & 75 & 25 & & & $7.5 \approx 8$ & 11 \\
\hline 3 & 100 & & & 50 & 50 & & & $6.5 \approx 7$ & 11.8 \\
\hline 4 & 100 & & & 25 & 75 & & & $8.3 \approx 8$ & 12 \\
\hline 5 & 100 & & 75 & 25 & & & & $3.8 \approx 4$ & 7.3 \\
\hline
\end{tabular}

Table D.7: Usability testing results - General questions - Non-multimodal

\begin{tabular}{|l|c|c|c|c|c|}
\hline \multicolumn{1}{|c|}{ Question } & \multicolumn{5}{c|}{$\%$} \\
\cline { 2 - 7 } & 1 & 2 & 3 & 4 & 5 \\
\hline \hline 1 Navigation difficulty level (low is good) & & 25 & 75 & & \\
\hline 2 Application structure (high is good) & & & & 100 & \\
\hline 3 Features in correct place (high is good) & & & 25 & 75 & \\
\hline & \multicolumn{2}{|c|}{ Yes } & \multicolumn{3}{|c|}{ No } \\
\hline 4 GUI easy to use & \multicolumn{2}{|c|}{25} & \multicolumn{3}{|c|}{75} \\
\hline 5 Confortable and confident with the application & \multicolumn{2}{|c|}{100} & \multicolumn{3}{|c|}{} \\
\hline
\end{tabular}

\section{D.3.3 Analysis of results}

Taking into account the results collected in pre and post-installation usability testing, a general improvement in the usability levels was observed. Although in both cases tasks were completed, the average time to complete a task was reduced in most tasks. For tasks without intermediate selection screens, the completion time was reduced to an interval of 5 to 6 seconds. Tasks that required user feedback to be completed required extra time because of normal interaction with the user. The second task, which had few clicks, showed a slight increase in the time (Figure D.8). If the level of difficulty of the task was relatively low in the non-multimodal test, these levels were reduced to one in the multimodal tests (Figure D.9). The most important result in the task-specific questions was the reduction of the number of clicks to one in tasks where user feedback is not necessary, and to two in tasks where an intermediate screen is presented to collect information to fulfill the task. These reduction resulted in usability improvements and in the reduction of the necessary focus on the device to fulfill a task. Subjects affirmed that navigation through the system is easier using speech recognition. There was a slight improvement in the perception of the structure of the application as well as the organization of the functionalites (Figure D.10). Comments 
Table D.8: Usability testing results - Specific questions - Multimodal

\begin{tabular}{|c|c|c||c|c|c|c|c||c|c|}
\hline & \multicolumn{7}{|c|}{ Questions } & $3^{+}$ & \\
\cline { 2 - 8 } & \multicolumn{2}{|c||}{$1^{*}$} & \multicolumn{7}{c|}{$2^{*}$} \\
\hline Tasks & Yes & No & 1 & 2 & 3 & 4 & 5 & Clicks & Time $^{\S}$ \\
\hline \hline 1 & 100 & & 100 & & & & & 1 & 5.3 \\
\hline 2 & 100 & & 100 & & & & & 1 & 5.5 \\
\hline 3 & 100 & & 100 & & & & & 1 & 5 \\
\hline 4 & 100 & & 100 & & & & & 2 & 8.3 \\
\hline 5 & 100 & 100 & & & & & 2 & 7.3 \\
\hline
\end{tabular}

* Percentages

+ Number of clicks

$\S$ Average in seconds

Table D.9: Usability testing results - General questions - Multimodal

\begin{tabular}{|c|c|c|c|c|c|}
\hline \multirow[t]{2}{*}{ Question } & \multicolumn{5}{|c|}{$\%$} \\
\hline & 1 & 2 & 3 & 4 & 5 \\
\hline 1 Navigation difficulty level (low is good) & 100 & & & & \\
\hline 2 Application structure (high is good) & & & & 100 & \\
\hline 3 Features in correct place (high is good) & & & & 75 & 25 \\
\hline & $\mathrm{Ye}$ & & & $\mathrm{N}$ & \\
\hline 4 Speech recognition easy to use & 10 & & & & \\
\hline 5 Confortable and confident with the application & 10 & & & & \\
\hline
\end{tabular}

from subjects suggested that speech recognition as an alternative input system was highly accepted because of the reduction of time and the recognition accuracy. Users also suggested including more commands in the vocabulary to access other functionalities and increasing the menu size to avoid tapping on adjacent elements. In general, users found the top-right corner easily accessible to activate speech recognition, although there was a suggestion to place the button at the botton-center of the application. After explaining the convention for speech decoding states, users easily identified the time to start speaking and when the decoder was collecting and processing utterances.

Pre- and post-multimodal test results

Specific question 3

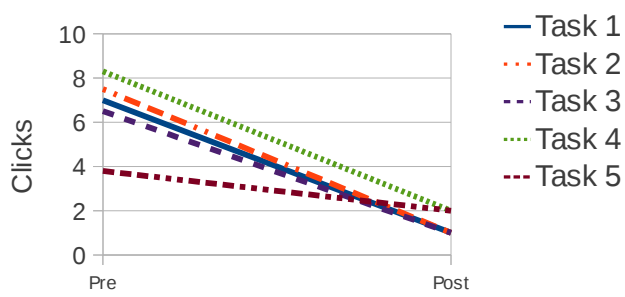

Pre- and post-multimodal results

Time used

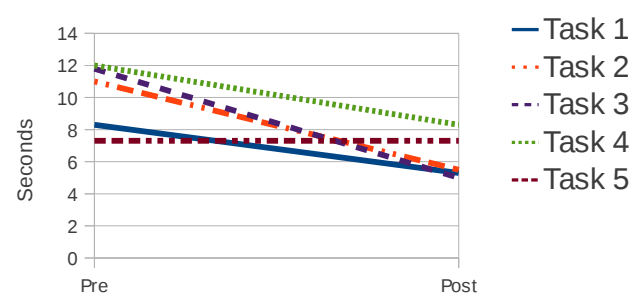

Figure D.8: Clicks and time reduction 
Pre-multimodal test results Specific question 2

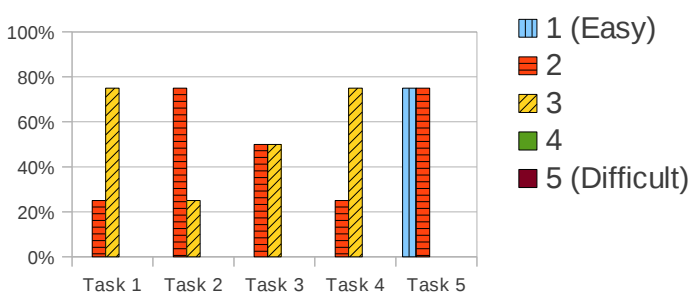

Post-multimodal test results Specific question 2

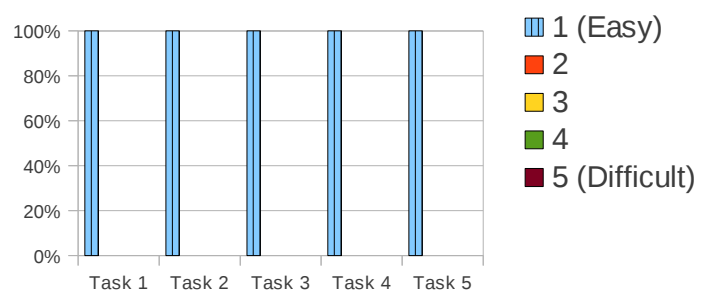

Figure D.9: Specific questions results

Pre-multimodal test results

General questions (Likert's scale)

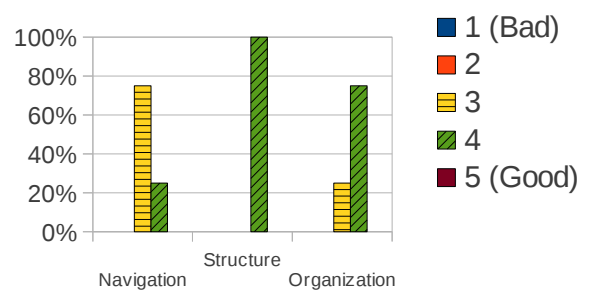

Post-multimodal test results

General questions (Likert's scale)

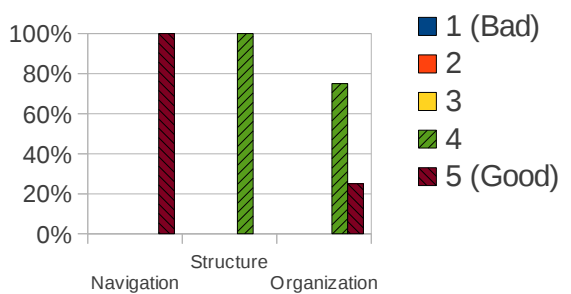

Figure D.10: General questions results 


\section{Appendix E}

\section{Discussion}

This chapter addresses the question of how this project contributed to the work on multimodal interfaces on mobile devices, whether the goals have been reached, and what are the directions for future research in this area. Conclusions of the project are presented and contrasted with the goals defined at the beginning of the project. Future directions are presented at the end of the chapter.

Using mobile devices in everyday-life have become very normal in cities and, at a lower level, in rural areas. Most urban dwellers have at least one mobile device (e.g., cellphones or handhelds) and they interact increasingly with devices embedded in appliances or cars. In the last decade these devices have evolved dramatically. Faster processors, more RAM, more storage capacity and multiple connection options have transformed simple mobile devices into tiny portable work stations. However, due to their portability requirements, these devices continue to be small. While screen resolutions increase, their sizes are still small. More graphical components are available to enrich mobile applications, but as before, mobile screens have to present simple interfaces to the users for usability. Current input modes are not enough for the current needs of mobility. Tiny qwerty keyboards are available on new devices, but the users are not always able to focus on visible and tangible device inputs or outputs. Multimodal interfaces (MMI) have opened a new research area for human-computer interaction. MMI allows people to use computers in a more natural way by extending common input/output methods, mixing them or using new ones to better address user needs. Since speaking is a natural way to communicate, researchers have faced the challenge of making computers able to understand user requests using voice, taking advantage of research advances in speech recognition. The use of statistical models and language processing methodologies have resulted in high accuracy levels in isolated and continuous recognition. On mobile devices speech recognition brings great benefits for the user interaction, extending the limited input modes to richer ones.

This work presents the use of speech recognition as a multimodal input method that seeks to improve the use of a data collection mobile application for medical visits, Borboleta, carried out under home care initiatives (Family Health and Homecare program) in the Brazilian health system. Usability improvements of the Borboleta system have the potential to result in better patient care during medical visits because of the reduced need for software attention. The objectives of the project were to verify if the use of speech recognition as input method improves the usability levels of a mobile application as Borboleta, to evaluate if current mobile devices are able to perform sophisticated tasks like utterance processing and decoding in acceptable time ranges without affect device performance or lifetime, and to contribute in research of speech recognition for Portuguese Brazilian language.

This document presented a review of the theoretical background for multimodal interfaces, speech recognition and mobile platforms. It also presented the methodology and development of the project and showed how a speech recognition system (Pocketsphinx) was integrated to 
the Borboleta application on a Symbian platform. Finally, it presented the experimental design to validate the different aspects of the project: software performance, speech recognition accuracy and system usability.

It was verified that for speech recognition accuracy levels for command recognition in Brazilian Portuguese are in an acceptable range for general-use applications. Also, the evaluation of four different approaches for command recognition clarified that the use of phonemebased word representation and individual word-based language modeling results in lower word error rates. However, all approaches resulting in similar command-level recognition rates with acoustic model topology yielding the most improvement in accuracy.

For software performance, the results showed that modern mobile devices are able to perform full speech recognition tasks for language and acoustic models of small sizes, like commands or numbers, in a very acceptable response time. For larger vocabularies, speech recognition on mobile devices could be implemented through two other approaches, distributed speech recognition, where features are extracted on mobile device and recognition is done on a external server, or network speech recognition, where the mobile phone sends the utterance to a external server to be processed and decoded. It was also verified that integration of $\mathrm{C}++$ and Java ME applications is a viable solution to use external functionalities based on Java without affecting device performance. Performance problems found during the experiments were attributed to the Java ME virtual machine that requires extra processing to execute applications.

In the usability field, this research contributed to the Borboleta project in its current work on mobile devices by extending the project to include multimodal interfaces. The use of speech recognition as an alternative input method gave the user the option to interact with the application in a more natural way. The user experience was improved, especially for novice users who were not familiar with the structure of the application. Because of low word error rates, the users accepted the speech recognition option easily, and in several cases this option was preferred. Users suggested including more vocabulary to manipulate the application. The multimodal interface was widely accepted because of the high recognition rate, easy access to the speech button, clear feedback to the user about the decoding process, and, most importantly, a reduction in the time used to manipulate the system.

Other contributions include the inclusion of the Pocketsphinx modifications into the official sources in the main development version of the project (trunk) available at the CMUSphinx site, ${ }^{1}$ the interest of Nokia (Brazil) in collaboration on SpeechMe development, the development of an application to collect audio samples, the construction of a basic command corpora for speech recognition for Brazilian Portuguese, and the possibility to bring the advances in speech recognition closer to the society in a developing country through new interaction paradigms.

\section{E.1 Future directions}

Since this research included work from different fields, there are several research paths to extend the topics examined in this work. There are important initiatives addressing speech recognition for Brazilian Portuguese like FalaBrasil, to develop robust speech recognition systems and high quality acoustic and language models. People who want to follow this research line could work on improving command recognition by adding larger vocabularies or including language processing modules that allow the user to request additional services to the system in more natural ways. In the healthcare field, continuous speech recognition could be used for transcribing medical notes recorded during medical visits, avoiding data input using conventional keyboards or touchscreens and reducing the focus on the device. Current mobile devices present good performance for speech processing on small vocabularies

\footnotetext{
${ }^{1}$ http://cmusphinx.sourceforge.net
} 
using embedded speech recognition approaches. However, it is still necessary to use remote services for recognition of large vocabularies.

Another field of research that would extend this work is the optimization of the current signal processing and word search algorithms to reduce the response time on mobile devices when using embedded approaches, giving support to larger vocabularies or increasing the battery life. For network and distributed recognition approaches, signal representation algorithms and communications protocols could be studied in order to reduced bandwidth usage and improve recognition response time. 


\section{Appendix F}

\section{Acoustic and Language modeling}

This appendix presents the phone strings used for representing word approach 2 presented in section C.4, which presented the best results in command recogntion (Table F.1). The FSG language model for individual word modeling used in approaches 1 and 2 is shown in Figure F.1.

Table F.1: Phonemes for Command words

\begin{tabular}{|c|c|}
\hline Word & Phones \\
\hline agenda & a $\mathrm{Z}$ e $\sim \mathrm{d}$ a \\
\hline agendar & a $Z$ e $\sim d a X$ \\
\hline caracterização & $\mathrm{k}$ araktarizas a $\sim \mathrm{w} \sim$ \\
\hline catálogo & $\mathrm{k}$ a t a log $\mathrm{u}$ \\
\hline cid & s i dZ \\
\hline dados & $\mathrm{d}$ a d u s \\
\hline $\mathrm{e}$ & $\mathrm{i}$ \\
\hline encontro & $\mathrm{e} \sim \mathrm{k} \mathrm{o} \sim \mathrm{tru}$ \\
\hline encontros & $\mathrm{e} \sim \mathrm{k} \mathrm{o} \sim \mathrm{trus}$ \\
\hline específicos & e s p e sifik us \\
\hline fechar & f e $\mathrm{S}$ a $\mathrm{X}$ \\
\hline final & $\mathrm{f} i \sim \mathrm{n}$ a $\mathrm{w}$ \\
\hline geral & $\mathrm{Z}$ e r a w \\
\hline inicial & $\mathrm{i} \sim \mathrm{n}$ i s i a w \\
\hline menu & $\mathrm{m}$ e $\mathrm{n} \mathrm{u}$ \\
\hline necessidades & n e s e s i d a dZ i s \\
\hline novo & $\mathrm{n} \mathrm{o} \mathrm{v} \mathrm{u}$ \\
\hline paciente & $\mathrm{p}$ a s i e $\sim \mathrm{tS} \mathrm{i}$ \\
\hline problemas & $\mathrm{p} \mathrm{roble} \sim \mathrm{m} \mathrm{a} \mathrm{s}$ \\
\hline programação & $\mathrm{p}$ r o g r a m a s a $\sim \mathrm{w} \sim$ \\
\hline realizados & R e a liza d u s \\
\hline sair & s a j X \\
\hline visita & v i z i t a \\
\hline voltar & vo w t a X \\
\hline
\end{tabular}


\#JSGF V1.0;

grammar commands;

public <Commands> = (DADOS ESPECIFICOS) | (CARACTERIZACAO GERAL) | (PROBLEMAS E NECESSIDADES) ।

(PROGRAMACAO FINAL) | (ENCONTROS REALIZADOS) |

(ENCONTRO) | (NOVO ENCONTRO) | (MENU INICIAL) |

(PACIENTE) | (AGENDA) | (AGENDAR VISITA) |

(CATALOGO CID) | (VOLTAR) | (FECHAR) | (SAIR);

Figure F.1: Language model for individual words 


\section{Appendix G}

\section{IDEs used during the project development}

This appendix presentes an overview of the development environments used during project development. Carbide.c ++ was used for Pocketsphinx porting and $\mathrm{C}++$ development, QtCreator was used for graphical interface design on $\mathrm{C}++$ side, and Netbeans was used for development of the Java Me side.

\section{G.1 Carbide.c ++}

Carbide.c++ is a Nokia IDE for Symbian C++ and Qt development based on Eclipse IDE. Thanks to its base, Carbide.c++ comes with an extensive tool set for mobile application development, including code editors, user interface designers, project management tools, debuggers, profilers, revision control support, and support for different compilers. The compiler version depends on the software development kit (SDK) installed. Commonly, it includes the WINSCW x86 C++ compiler for Symbian emulator and the GCC compiler for ARM targets. There is an additional ARM RVCT compiler, which is sold separately and includes compilation optimizations for a higher performance.

For the current project, Carbide.c ++ 2.6.0 and the Nokia N97 SDK were used which include a full Nokia N97 emulator. For installation, an x86-based computer with $4 \mathrm{~Gb}$ of RAM with Windows XP as operating system was used. To get a full-configured development environment for Symbian the following applications were installed in the listed order:

1. Nokia OVI suite

2. ActivePerl-5.6.1.635

3. Nokia N97 SDK

4. CSL ARM toolchain

5. Carbide.c ++2.3 .0 from Symbian Foundation, updated to Carbide.c ++2.6 .0$

It is important to highlight that the tool froms 2 onwards must be installed in the same disk partition to avoid compilation errors.

In order to create a Symbian project a Carbide wizard is used (Figure G.1). Here, the basic structure of the project is created, including folder organization and configuration files, below is presented a typical structure of a Symbian project.

- inc folder: Includes the own header files.

- $\operatorname{src}$ folder: Keeps the source files. 


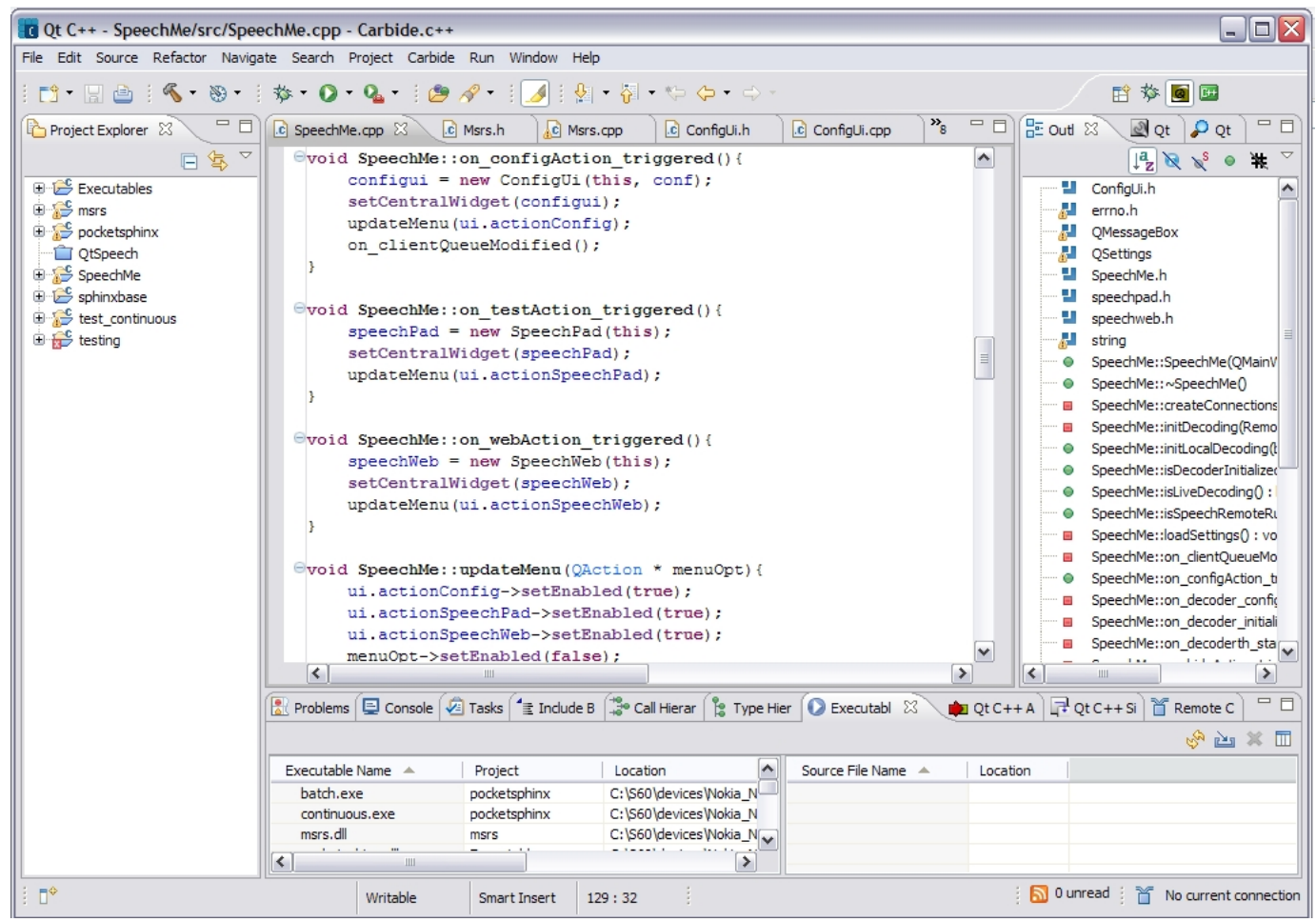

Figure G.1: Carbide IDE - New project wizard

- group folder: Contains the build files (mmp, pkg and bld)

- data folder: Place for resource files like images or sounds.

- sis folder: It is used when the installer package is created, maintains the sis and sisx files.

This folder configuration is not mandatory, but it is recommended to use this in order to have easier understanding during code evaluation or modification. In order to link, compile and package the application, three files contained in the group folder are necessary [64]. The MMP file contains the configuration of the Symbian application, its type, name, UIDs, target, headers, source files, library dependencies, and others. The Bld.inf file contains the MMP files that must be included during the building. The PKG file describes how the SIS file must be package to be installed in the device. The SISX file is a signed version of the package. An important tool is the Symbian emulator which is included in the SDK. The emulator provides most of the Symbian device features, but the building process is carried out using the WINSCW compiler. Figure G.2 shows the Nokia N97 emulator used during the project development.

\section{G.2 QtCreator}

QtCreator is a cross-platform IDE, currently part of the Nokia development tools. This IDE gives support to Qt applications that include graphical user interfaces and $\mathrm{C}++$ in general is also supported. QtCreator is composed of a $\mathrm{C}++$ and Javascript editor, a UI designer, a project management system, version control support, and a Qt simulator. It is be able to build desktop and mobile applications. Figure G.3 shows a QtCreator screen. For mobile development QtCreator can be used regardless of the Carbide.c++ for pure Qt/C++ applications. In the case of developing a native Symbian application that includes Qt features, for example graphical interfaces, the QtCreator can be used embedded into the Carbide 


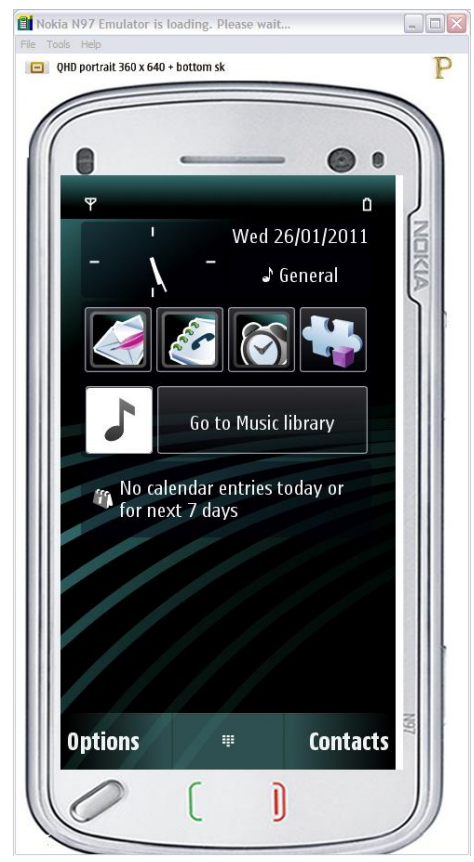

Figure G.2: Symbian emulator

IDE. For this project, Pocketsphinx porting was done directly in the Carbide IDE, because it does not include any Qt feature. For the MSRS application, Qt was used for the user interface design and for the socket communication. The version used for the development was QtCreator 2.6 with Qt 4.7.0.

\section{G.3 Netbeans}

Netbeans is an open-source IDE that supports multiple programming languages. Developed in Java, Netbeans has a complete set of software development tools. J2SE, J2EE and Java ME application development is supported as well as $\mathrm{C} / \mathrm{C}++$, Python, Ruby, PHP, Javascript, and others. Netbeans includes support for debugging, revision control and team working. In order to reduce compatibility problems in the Borboleta project Ant was used as a building system. Thus, the application could be configured with any IDE with mobile development support. Here, Netbeans 6.9.1 was used without affecting the compatibility constraints. To create Java mobile applications it is necessary to install the wireless toolkit (WTK) from Oracle. This toolkit provides the required libraries to compile Java ME applications and includes a phone emulator to test the application. In order to build the Borboleta project using Netbeans the following tools were installed:

- Java SDK 1.5.

- WTK 2.5 .

- Netbeans 6.9.1 with Java ME support.

It is necessary that the Java SDK be installed prior to Netbeans. Figure G.4 shows a typical window while developing using Netbeans. Figure G.5 shows the Java ME emulator provided by WTK. 


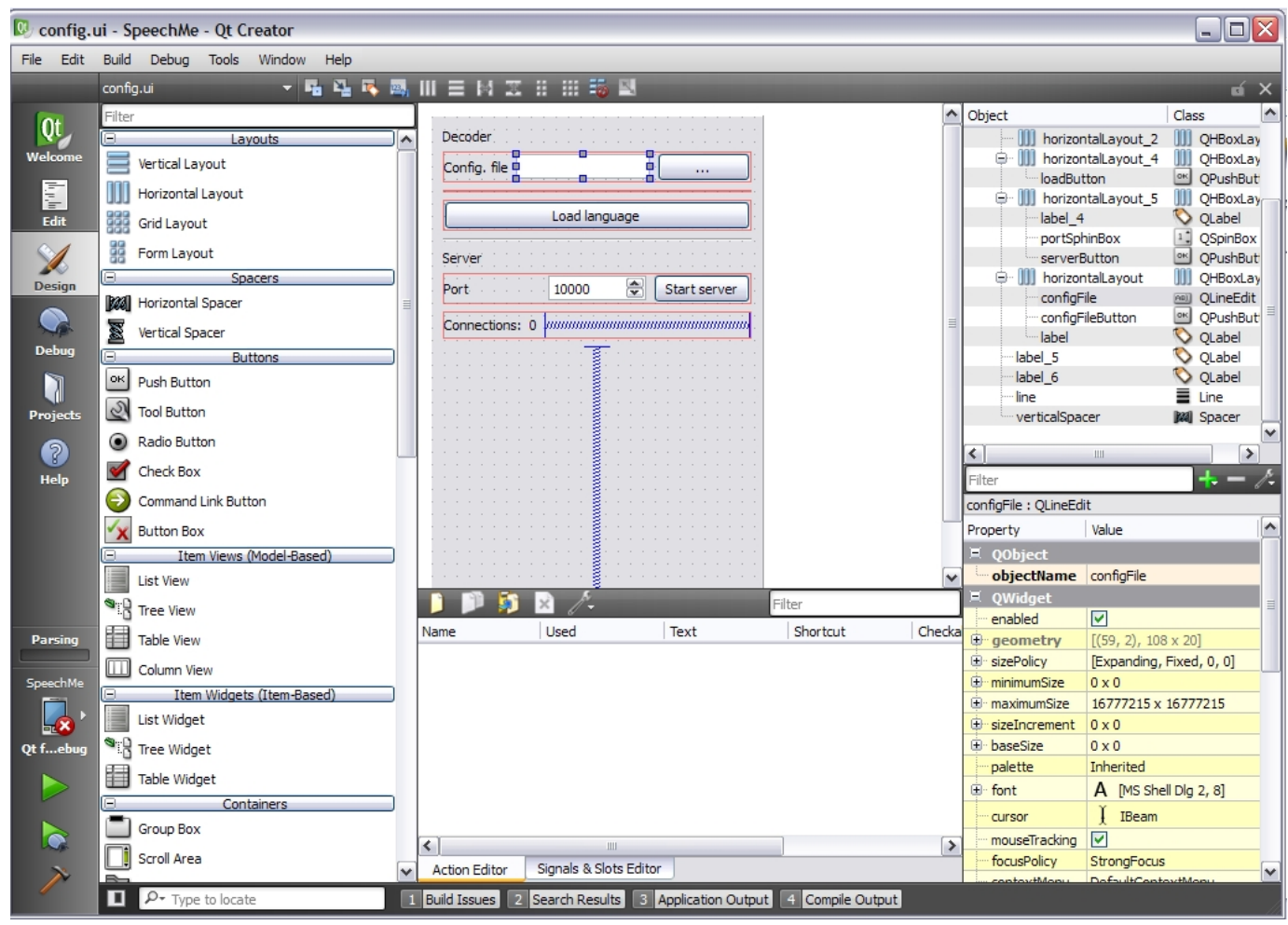

Figure G.3: QtCreator IDE

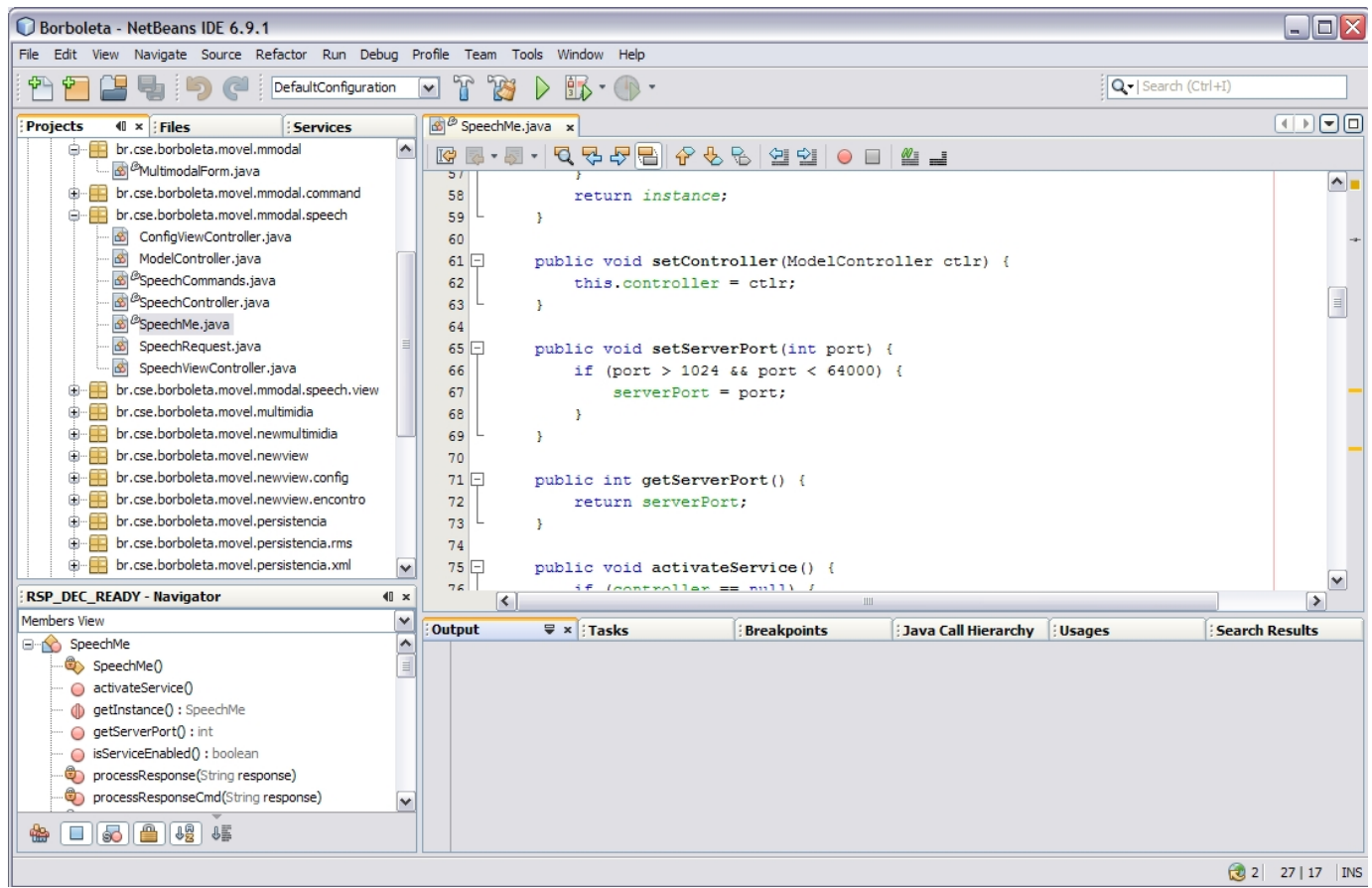

Figure G.4: Netbeans IDE 


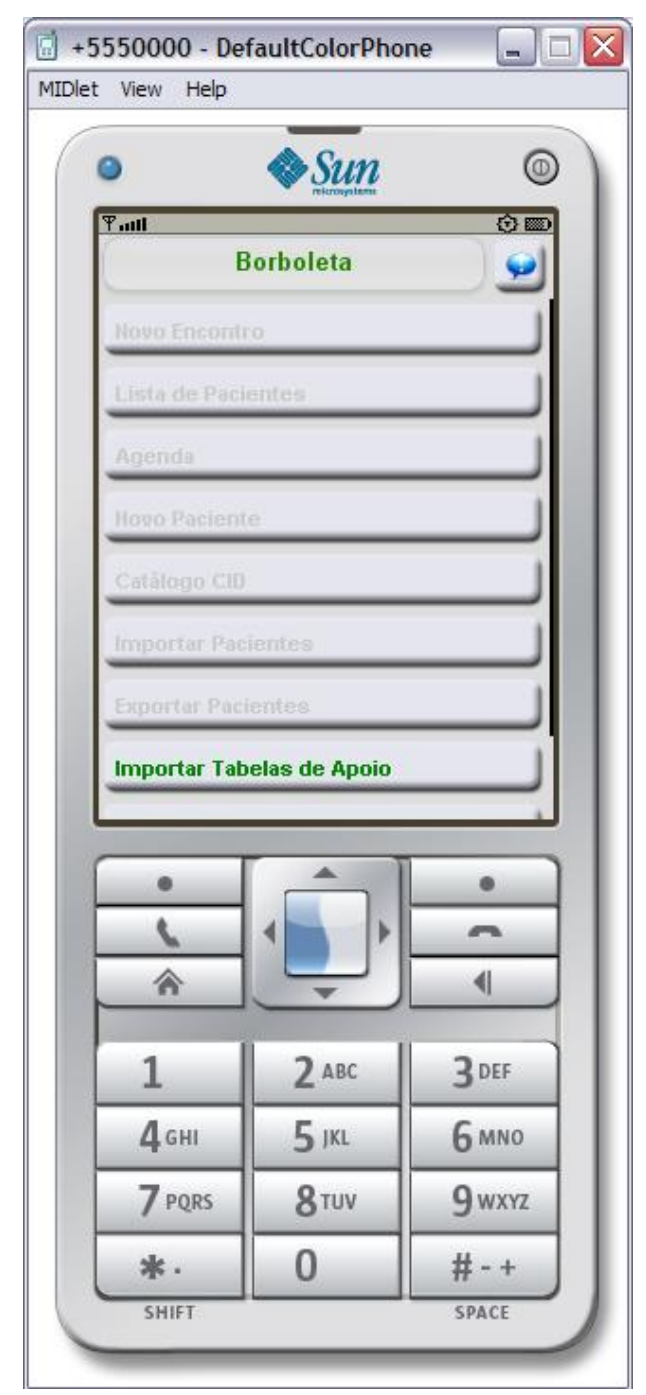

Figure G.5: Java ME emulator 


\section{Appendix $\mathbf{H}$}

\section{MMP files for Sphinxbase and Pocketsphinx}

Here is presented the building configuration files for compiling Pocketsphinx for the Symbian platform. Since the decoder is composed of two projects, two files were used to compile the application, one to create the Sphinxbase library and the other to create the Pocketsphinx library. Two additional files for con

figuration, the pocketsphinx batch and pocketsphinx continuous executable, were also created but these have similar structure to those presented here.

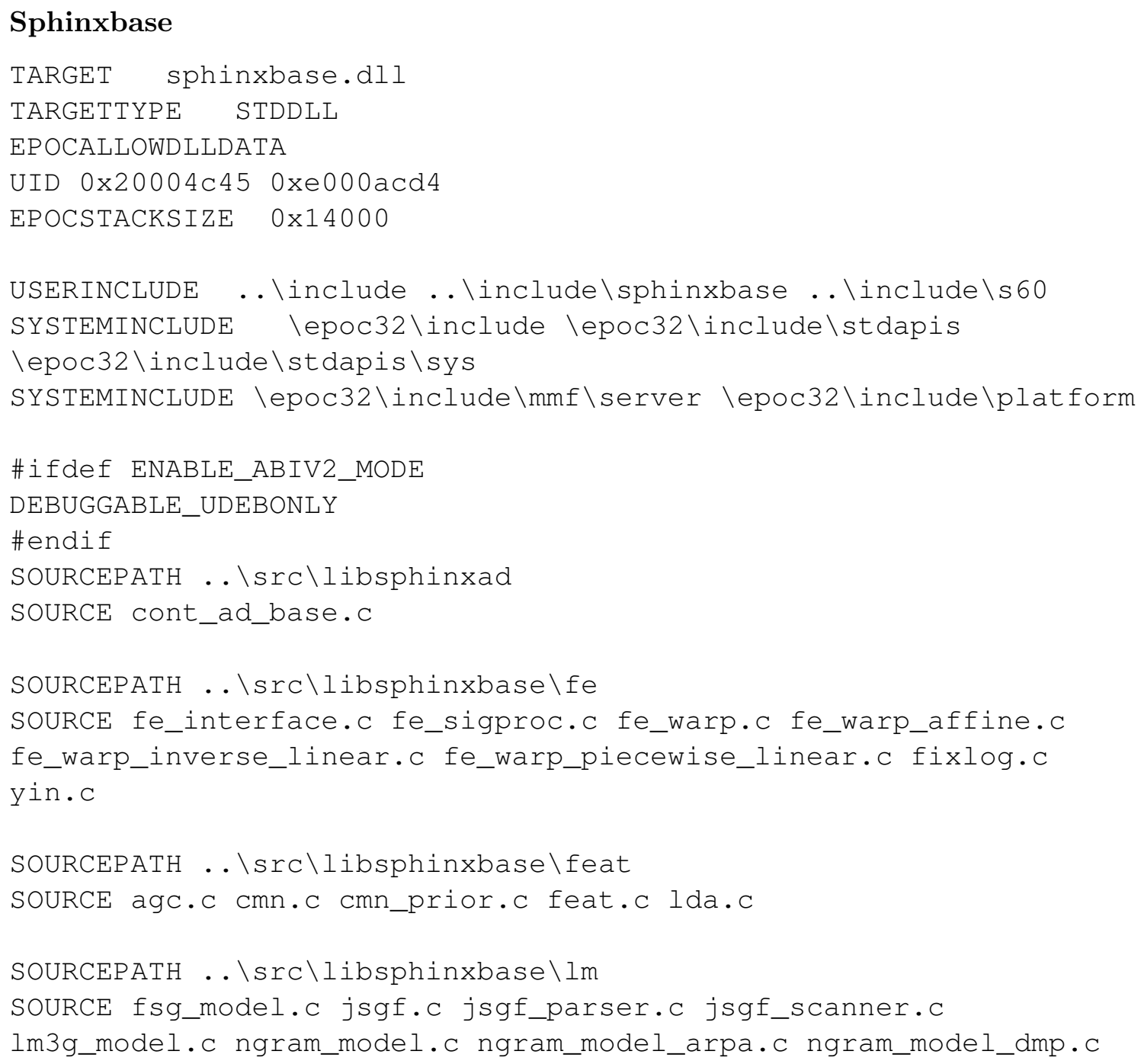


ngram_model_dmp32.c ngram_model_set.c

SOURCEPATH ..\srC\libsphinxbase \util

SOURCE bio.c bitvec.c blas_lite.c case.c ckd_alloc.c cmd_ln.c

dtoa.c err.c f2c_lite.c filename.c genrand.c glist.c hash_table.c heap.c h uff_code.c info.c listelem_alloc.c logmath.c matrix.c mmio.c pio.c profile.c sbthread.c slamch.c slapack_lite.c

strfuncs.c unlimit.c utf8.c

SOURCEPATH $\ldots \backslash$ srC $\backslash$ libsphinxad

SOURCE ad_s60.cpp

\section{Pocketsphinx}

TARGET pocketsphinx.dll

TARGETTYPE STDDLL

UID 0x20004C45 0xe000acd3

EPOCALLOWDLLDATA

EPOCSTACKSIZE OX14000

USERINCLUDE ..\include ..\src\libpocketsphinx

$\ldots . . \backslash$ sphinxbase \include \s60 ..\..

.. . . \sphinxbase \include

SYSTEMINCLUDE \epoc32\include \epoc32\include \stdapis

$\backslash$ epoc32\include \stdapis\sys

SOURCEPATH . . SsrC\libpocketsphinx

SOURCE acmod.c bin_mdef.c blkarray_list.c dict2pid.c dict.c

fsg_history.c fsg_lextree.c fsg_search.c hmm.c molef.c ms_gauden.c ms_mgau.c ms_senone.c ngram_search.c ngram_search_fwdflat.c

ngram_search_fwdtree.c phone_loop_search.c pocketsphinx.c

ps_lattice.c ps_mllr.c ptm_mgau.c s2_semi_mgau.c tmat.c vector.c

LIBRARY libm.lib sphinxbase.lib

CAPABILITY UserEnvironment

\#ifdef ENABLE_ABIV2_MODE

DEBUGGABLE_UDEBONLY

\#endif 


\section{Appendix I}

\section{Protocol communication values}

This appendix specifies the protocol values for using the speech recognition service from an external application using socket communication. Table I.1 presents the states of the decoder. Table I.2 contains the response type for control, decoding status and decoded sentence. Table I.3 presents the messages for communication through sockets

Table I.1: Decoding states

\begin{tabular}{|c|l|c|}
\hline State & \multicolumn{1}{|c|}{ Meaning } & Value \\
\hline CONFIGURED & The language model has been loaded & 0 \\
\hline INITIALIZED & Pocketsphinx is ready to accept decoding request & 1 \\
\hline READY & The user can start speaking & 2 \\
\hline LISTENING & The system is collecting utterances & 3 \\
\hline PROCESSING & The system is decoding the utterance & 4 \\
\hline STOPPED & Decoding finished & 5 \\
\hline FAIL & Error during decoding & 99 \\
\hline
\end{tabular}

Table I.2: Response control values - prefixes

\begin{tabular}{|c|l|c|}
\hline Variable & \multicolumn{1}{|c|}{ Meaning } & Value \\
\hline RSP_CMD_PREFIX & Indicates a notification of connection status & rsp_cmd \\
\hline RSP_DEC_PREFIX & Indicates a notification for decoding status & rsp_dec \\
\hline No prefix & $\begin{array}{l}\text { If no prefix is in the received string, the } \\
\text { string is a decoded sentence }\end{array}$ & Sentence \\
\hline
\end{tabular}


Table I.3: Decoding states

\begin{tabular}{|c|l|c|}
\hline \multicolumn{1}{|c|}{ State } & \multicolumn{1}{|c|}{ Meaning } & Value \\
\hline RSP_DEC_CONFIGURED & Decoder configured & rsp_dec_conf \\
\hline RSP_DEC_INITIALIZED & Decoder initialized & rsp_dec_init \\
\hline RSP_DEC_READY & Decoder ready to capture utterances & rsp_dec_ready \\
\hline RSP_DEC_LISTENING & Collecting utterances & rsp_dec_lst \\
\hline RSP_DEC_PROCESSING & Decoding & rsp_dec_proc \\
\hline RSP_DEC_STOPPED & Decoding finished & rsp_dec_stop \\
\hline RSP_DEC_FAIL & Error during decoding & rsp_dec_fail \\
\hline RSP_CMD_REG_OK & Client registry successfull & rsp_cmd_reg_ok \\
\hline RSP_CMD_REG_FAIL & Client registry failed & rsp_cmd_reg_fail \\
\hline RSP_CMD_REM_OK & Remove client successfull & rsp_cmd_rem_ok \\
\hline RSP_CMD_REM_FAIL & Remove client fail & rsp_cmd_rem_fail \\
\hline
\end{tabular}




\section{Appendix J}

\section{Usability questionnaires}

Figure J.1 shows the questionnaire used in the pre-installation testing in English (Nonmultimodal). Questionnaire of post-installation test only is changed the general question 4 to ask about the easiness of using speech recognition. Figure 4.2 shows the pre-installation questionnaire in Portuguese.

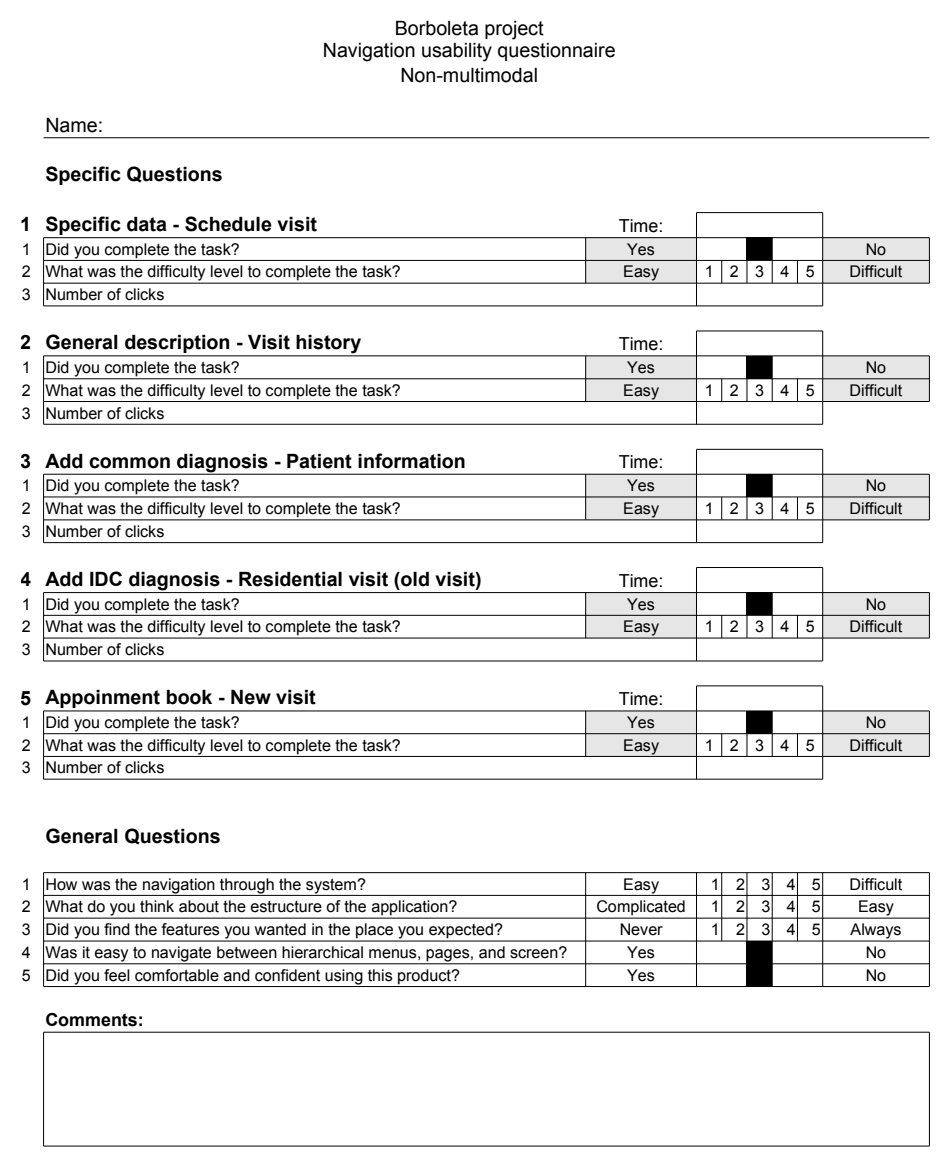

Suggestions:

Figure J.1: Pre-installation usability questionnaire - English 


\section{Referências Bibliográficas}

[1] Hermann Anegg, Georg Niklfeld, Raimund Schatz, Rainer Simon, and Florian Wegscheider. Multimodal interfaces in mobile devices - the MONA project. In MobEA II Emerging Applications for Wireless and Mobile Access Workshop at the 13th International World Wide Web Conference, 2004. 11, 53

[2] A. D. Baddeley and G. J. Hitch. Working memory. In G. A. Bower, editor, Recent Advances in Learning and Motivation, volume 8. Academic Press, 1974. 6, 37

[3] J. K. Baker. Stochastic modeling for automatic speech understanding. In D. R. Reddy, editor, Speech Recognition, pages 521-542. Academic Press, New York, 1975. 38, 44

[4] Rebecca Anne Bates. Speaker Dynamics as a Source of Pronunciation Variability for Continuous Speech Recognition Models. PhD thesis, University of Washington, 2003. 6, 40

[5] Cambridge University Engineering Department. HTK - Hidden Markov Model Toolkit - http://htk.eng.cam.ac.uk, April 2010. 39

[6] Luca Chittaro. Distinctive aspects of mobile interaction and their implications for the design of multimodal interfaces. Journal on Multimodal User Interfaces, 3:157-165, 2010. 10.1007/s12193-010-0036-2. 37

[7] Y. L. Chow, M. O. Dunham, O. A. Kimball, M. A. Krasner, G. F. Kubala, J. Makhoul, P. J. Price, S. Roucos, and R. M. Schwartz. BYBLOS: the BBN continuous speech recognition system. pages 596-599, 1990. 39

[8] CMU. CMU Sphinx Wiki - http://cmusphinx.sourceforge.net/wiki, May 7 2010. 39

[9] M. Cohen, H. Murveit, J. Bernstein, P. Price, and M. Weintraub. The DECIPHER speech recognition system. In International Conference on Acoustics, Speech, and Signal Processing, pages $77-80$ vol.1, apr 1990. 39

[10] Douglas E. Comer and David L. Stevens. Internetworking with TCP/IP, Vol. III: ClientServer Programming and Applications, Linux/Posix Sockets Version. Prentice Hall, US ed edition, September 2000. 72

[11] IBM Corporation and Microsoft Corporation. Multimedia programming interface and data specifications 1.0 - http://www.tactilemedia.com/info/mci_control_info.html, August 1991. 15, 58

[12] Rafael Correia, Arlindo Flávio da Conceição, Fabio Kon, Rubens Kon, and José Ricardo Brandão. Sistema móvel multimídia de código aberto para atenção primária de saúde com visitas domiciliares. 10th Workshop on Free Software, 2009. 49

[13] Rafael Correia, Fabio Kon, and Rubens Kon. Borboleta: A mobile telehealth system for primary homecare. 23rd ACM Symposium on Applied Computing, 2008. 2, 8, 33 
[14] CSLU and OGI. 22 Language v1.3 - http://cslu.cse.ogi.edu/corpora/22lang/, April 2010. 8,46

[15] CSLU and UFRGS. The Spoltech Brazilian Portuguese v1.0 - http://cslu.cse.ogi.edu/ corpora/spoltech, April 2010. 8, 46

[16] Helves Domingues, Rafael José Peres Correia, Fabio Kon, Eduardo Ferreira, and Rubens Kon. Análise e modelagem conceitual de um sistema de prontuário eletrônico para centros de saúde. WIM - Workshop de Informática Médica, 2008. 46

[17] G. L. Duarte, R. Correia, P. Leal, H. Domingues, F. Kon, R. Kon, and J. E. Ferreira. Borboleta and SAGUISaúde - Open Source Mobile Telehealth for Public Home Healthcare. In Proc. 8th International eHealth, Telemedicine and Health ICT Forum (Med-e-Tel), 2010. 8

[18] Homer Dudley and T. H. Tarnoczy. The Speaking Machine of Wolfgang von Kempelen. The Journal of the Acoustical Society of America, 22(2):151-166, 1950. 38

[19] Bruno Dumas, Denis Lalanne, and Sharon Oviatt. Multimodal interfaces: a survey of principles, models and frameworks. In Denis Lalanne and Jürg Kohlas, editors, Human Machine Interaction, volume 5440 of Lecture Notes in Computer Science, pages 3-26. Springer Berlin / Heidelberg, 2009. 5, 6, 36, 37

[20] G. Evermann, H.Y. Chan, M.J.F. Gales, B. Jia, D. Mrva, P.C. Woodland, and K. Yu. Training LVCSR systems on thousands of hours of data. ICASSP, I:209-212, 2005. 7, 8,40

[21] Nokia Forum. Midlet native services framework - http://wiki.forum.nokia.com/ index.php/midletnativeservicesframework, March 2010. Accessed Feb. 2011. 72

[22] Erich Gamma, Richard Helm, Ralph E. Johnson, and John Vlissides. Design Patterns: Elements of Reusable Object-Oriented Software. Addison-Wesley, Reading, MA, 1995. $18,67,69,77$

[23] Gartner. Worldwide Smartphone Sales to End Users by Operating System in 1Q11 http://www.gartner.com/it/page.jsp?id=1689814, May 2011. 48

[24] I. L. Hetherington. PocketSUMMIT: small footprint continuous speech recognition. INTERSPEECH 200\%. 11, 13, 51, 56

[25] John Holmes and Wendy Holmes. Speech Synthesis and Recognition. Taylor \& Francis, Inc., Bristol, PA, USA, 2002. 42, 43, 44

[26] J. Huang, V. Goel, R. A. Gopinath, B. Kingsbury, P. Olsen, and K. Visweswariah. Large vocabulary conversational speech recognition with the extended maximum likelihood linear transformation (EMLLT) model. ICSLP, 2002. 7, 8, 40

[27] Xuedong Huang, Alex Acero, and Hsiao-Wuen Hon. Spoken Language Processing: A Guide to Theory, Algorithm, and System Development. Prentice Hall PTR, Upper Saddle River, NJ, USA, 2001. 7, 15, 39, 40, 41, 45, 58

[28] Xuedong Huang, Fileno Alleva, Mei-Yuh Hwang, and Ronald Rosenfeld. An overview of the SPHINX-II speech recognition system. In HLT '93: Proceedings of the Workshop on Human Language Technology, pages 81-86, Morristown, NJ, USA, 1993. 10, 39, 50

[29] D. Huggins-Daines. PocketSphinx: a free real-time continuous speech recognition system for hand-held devices. Proc. ICASSP 2006, pages 185-188, 2006. 10, 12, 13, 39, 50, 53, 56 
[30] IBM. Via Voice - http://www-01.ibm.com/software/pervasive/embedded_viavoice, April 2010. 39

[31] Dale Isaacs and Daniel J. Mashao. A tutorial on distributed speech recognition for wireless mobile devices. Southern African Telecommunications and Applications Conference (SATNAC 2007), pages 9-13, 2007. 4, 35

[32] R. K. Jain. The Art of Computer Systems Performance Analysis: Techniques for Experimental Design, Measurement, Simulation, and Modeling. Wiley, 1st edition, April 1991. 79

[33] B.H. Juang and Lawrence R. Rabiner. Automatic speech recognition - a brief history of the technology development. Elsevier Encyclopedia of Language and Linguistics, 2nd Edition, 2005. 38

[34] Tomonari Kamba, Shawn A. Elson, Terry Harpold, Tim Stamper, and Piyawadee Sukaviriya. Using small screen space more efficiently. In CHI '96: Proceedings of the SIGCHI Conference on Human Factors in Computing Systems, pages 383-390, New York, NY, USA, 1996. ACM. 2, 33

[35] H. Kokubo, N. Hataoka, A. Lee, T. Kawahara, and K. Shikano. Embedded Julius: continuous speech recognition software for microprocessor. In 8th Workshop on Multimedia Signal Processing, 2006 IEEE, pages 378 -381, October 2006. 39

[36] Kai-Fu Lee, Hsiao-Wuen Hon, Sanjoy Mahajan Hwang, and Raj Reddy. The Sphinx speech recognition system. In HLT '89: Proceedings of the Workshop on Speech and Natural Language, pages 125-130, Morristown, NJ, USA, 1989. 39

[37] Kwang Lee and Roger Grice. The design and development of user interfaces for voice application in mobile devices. In International Professional Communication Conference, 2006 IEEE, pages $308-320,2006.11,52$

[38] Sheng Liang. Java Native Interface: Programmer's Guide and Reference. AddisonWesley Longman Publishing Co., Inc., Boston, MA, USA, 1st edition, 1999. 71

[39] Rensis Likert. A technique for the measurement of attitudes. Archives of Psychology, 140:1-55, 1932. 86

[40] University College London. Speech Assessment Methods Phonetic Alphabet, http://www.phon.ucl.ac.uk/home/sampa, April 2010. 58, 59

[41] T. B. Martin, A. L. Nelson, and H.J. Zadell. Speech recognition by feature abstraction techniques. Technical report, Air Force Avionics Lab, 1964. 38

[42] Nagoya Institute of Technology. Open-Source Large Vocabulary CSR Engine Julius http://julius.sourceforge.jp, April 2010. 39

[43] Nelson Neto, Carlos Patrick, Aldebaro Klautau, and Isabel Trancoso. Free tools and resources for brazilian portuguese speech recognition. Journal of the Brazilian Computer Society, 17:53-68, 2011. 10.1007/s13173-010-0023-1. 8

[44] Nelson Neto, Patrick Silva, Aldebaro Klautau, and Andre Adami. Spoltech and OGI-22 baseline systems for speech recognition in brazilian portuguese. International Conference on Computational Processing of Portuguese Language, 5190/2008:256-259, 2008. 4, 34, 46,58 
[45] Nuance. Dragon NaturallySpeaking Solutions - http://www.nuance.com/naturallyspeaking, April 2010. 39

[46] Sharon Oviatt. The Human Computer Interaction Handbook: Fundamentals, Evolving Technologies and Emerging Applications. chapter Multimodal interfaces, pages 413-432.

L. Erlbaum Associates Inc., Hillsdale, NJ, USA, 2003. 5, 6, 36, 37

[47] Sharon Oviatt and Rebecca Lunsford. Multimodal interfaces for cell phones and mobile technology. International Journal of Speech Techonology, 8(2):127-132, June 2005. 2, $3,33,34$

[48] P. Placeway, S. Chen, M. Eskenazi, U. Jain, V. Parikh, B. Raj, M. Ravishankar, R. Rosenfeld, K. Seymore, M. Siegler, R. Stern, and E. Thayer. The 1996 Hub-4 Sphinx-3 system. In Proc. of DARPA Speech Recognition Workshop, 1996. 39, 50

[49] Lawrence Rabiner and Biing-Hwang Juang. Fundamentals of Speech Recognition. Prentice-Hall, Inc., Upper Saddle River, NJ, USA, 1993. 7, 40, 41

[50] Leah M. Reeves, Jennifer Lai, James A. Larson, Sharon Oviatt, T. S. Balaji, Stéphanie Buisine, Penny Collings, Phil Cohen, Ben Kraal, Jean-Claude Martin, Michael McTear, TV Raman, Kay M. Stanney, Hui Su, and Qian Ying Wang. Guidelines for multimodal user interface design. Comm. ACM, 47:57-59, January 2004. 37

[51] Steve Renals. Speech Recognition. COM3140 (Speech Technology) Course Notes, Department of Computer Science, University of Sheffield, 1998. 42

[52] Young Sam Ryu. Development of Usability Questionnaires for Electronic Mobile Products and Decision Making Methods. PhD thesis, Virginia Polytechnic Institute and State University, 2005. 23, 88

[53] T. Sakai and S. Doshita. Phonetic typewriter. The Journal of the Acoustical Society of America, 33(11):1664-1664, 1961. 38

[54] Alexander Schmitt, Dmitry Zaykovskiy, and Wolfgang Minker. Speech recognition for mobile devices. Int J Speech Technol, 11:63-72, 2008. 2, 7, 8, 33, 39, 40

[55] Marcos Serrano, Laurence Nigay, Rachel Demumieux, Jérôme Descos, and Patrick Losquin. Multimodal interaction on mobile phones: development and evaluation using ACICARE. In Proceedings of the 8th Conference on Human-Computer Interaction with Mobile Devices and Services, MobileHCI '06, pages 129-136, New York, NY, USA, 2006. ACM. 11, 53

[56] Helen Sharp, Yvonne Rogers, and Jenny Preece. Interaction Design: Beyond HumanComputer Interaction. Wiley, 2nd edition, March 2007. 85, 86

[57] Patrick Silva, Pedro Batista, Nelson Neto, and Aldebaro Klautau. Computational Processing of the Portuguese Language, chapter An open-source speech recognizer for brazilian portuguese with a windows programming interface, pages 128-131. Springer Berlin / Heidelberg, 2010. 39

[58] Oracle Sun. Java micro edition - http://java.sun.com/javame, May 2010. 49

[59] Spoken Language Systems. SUMMIT - http://groups.csail.mit.edu/sls/technologies/ asr.shtml, May 2010. 11, 51

[60] Bálint Toth and Géza Nemeth. Challenges of creating multimodal interfaces on mobile devices. In ELMAR, 200\%, pages 171 -174, 2007. 5, 36 
[61] Karlsruhe University. GlobalPhone Portuguese (Brazilian) - http://www.elda.org/ catalogue/en/speech/s0201.html, May 2010. 8, 46

[62] Keith Vertanen and Per Ola Kristensson. Parakeet: a continuous speech recognition system for mobile touch-screen devices. In IUI '09: Proceedings of the 13th international conference on Intelligent user interfaces, pages 237-246, New York, NY, USA, 2009. ACM. 11, 12, 39, 51, 53, 54

[63] VoxForge. VoxForge - http://www.voxforge.org, April 2010. 8, 46

[64] Steffen Wachenfeld, Markus Madeja, and Xiaoyi Jiang. Developing mobile multimedia applications on symbian os devices. In Xiaoyi Jiang, Matthew Ma, and Chang Chen, editors, Mobile Multimedia Processing, volume 5960 of Lecture Notes in Computer Science, pages 238-263. Springer Berlin / Heidelberg, 2010. 10.1007/978-3-642-12349-8_14. 98

[65] Willie Walker, Paul Lamere, Philip Kwok, and Bhiksha Raj. Sphinx-4: A flexible open source framework for speech recognition. Technical report, Sun Microsystems Laboratories, 2004. 2, 33, 39

[66] Nokia Forum Wiki. S60 3rd edition: Application development - http://wiki.forum. nokia.com/index.php/s60_3rd_edition:_application_development, May 2010. 49

[67] Mark Wilcox. Porting to the Symbian Platform. Wiley, 2009. 16, 50, 61, 62

[68] Steve Young, Gunnar Evermann, Mark Gales, Thomas Hain, Dan Kershaw, Xunying Liu, Gareth Moore, Julian Odell, Dave Ollason, Dan Povey, Valtcho Valtchev, and Phil Woodland. The HTK Book for HTK V2.0. Cambridge University Press, 1995. 2, 33, 44,45

[69] Dmitry Zaykovskiy. Survey of the speech recognition techniques for mobile devices. In 11th International Conference on Speech and Computer (SPECOM), pages 88-93, St. Petersburg (Russia), June 2006. 10, 50

[70] Dmitry Zaykovskiy and Alexander Schmitt. Java to micro edition front-end for distributed speech recognition systems. In The 2007 IEEE International Symposium on Ubiquitous Computing and Intelligence (UCI'07), Niagara Falls (Canada), 2007. 4, 35, 50

[71] Rui Zhang, Stephen North, and Eleftherios Koutsofios. A comparison of speech and GUI input for navigation in complex visualizations on mobile devices. In Proceedings of the 12th International Conference on Human Computer Interaction with Mobile Devices and Services, MobileHCI '10, pages 357-360, New York, NY, USA, 2010. ACM. 12, 17, $53,54,66$ 